\title{
Comparative host-coronavirus protein interaction networks reveal pan-viral disease mechanisms
}

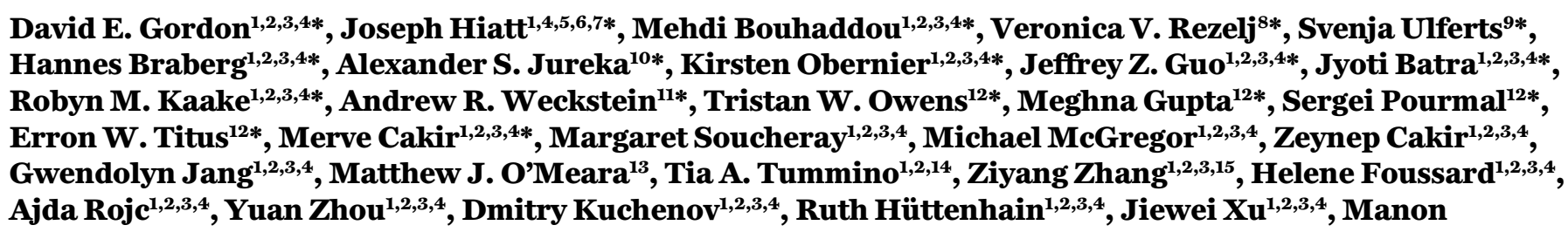
Eckhardt $^{1,2,3,4}$, Danielle L. Swaney ${ }^{1,2,3,4}$, Jacqueline M. Fabius ${ }^{1,2}$, Manisha Ummadi ${ }^{1,2,3,4}$, Beril Tutuncuoglu1,2,3,4, Ujjwal Rathore $^{1,2,3,4}$, Maya Modak ${ }^{1,2,3,4}$, Paige Haas, ${ }^{1,2,3,4}$, Kelsey M. Haas ${ }^{1,2,3,4}$, Zun Zar Chi Naing, ${ }^{1,2,3,4}$, Ernst H. Pulido $^{1,2,3,4}$, Ying Shi ${ }^{1,2,3,15}$, Inigo Barrio-Hernandez ${ }^{16}$, Danish Memon ${ }^{16}$, Eirini Petsalaki ${ }^{16}$, Alistair Dunham ${ }^{16}$, Miguel Correa Marrero ${ }^{16}$, David Burke ${ }^{16}$, Cassandra Koh ${ }^{8}$, Thomas Vallet ${ }^{8}$, Jesus A. Silvas ${ }^{10}$, Caleigh M. Azumaya $^{12}$, Christian Billesbølle ${ }^{12}$, Axel F. Brilot ${ }^{12}$, Melody G. Campbell ${ }^{12,17}$, Amy Diallo ${ }^{12}$, Miles Sasha Dickinson $^{12}$, Devan Diwanji ${ }^{12}$, Nadia Herrera ${ }^{12}$, Nick Hoppe ${ }^{12}$, Huong T. Kratochvil ${ }^{12}$, Yanxin Liu ${ }^{12}$, Gregory E. Merz $^{12}$, Michelle Moritz ${ }^{12}$, Henry C. Nguyen ${ }^{12}$, Carlos Nowotny ${ }^{12}$, Cristina Puchades ${ }^{12}$, Alexandrea N. Rizo ${ }^{12}$, Ursula Schulze-Gahmen ${ }^{12}$, Amber M. Smith ${ }^{12}$, Ming Sun ${ }^{12,18}$, Iris D. Young ${ }^{12}$, Jianhua Zhao ${ }^{12}$, Daniel Asarnow ${ }^{12}$, Justin Biel $^{12}$, Alisa Bowen ${ }^{12}$, Julian R. Braxton ${ }^{12}$, Jen Chen ${ }^{12}$, Cynthia M. Chio ${ }^{12}$, Un Seng Chio ${ }^{12}$, Ishan Deshpande $^{12}$, Loan Doan ${ }^{12}$, Bryan Faust ${ }^{12}$, Sebastian Flores ${ }^{12}$, Mingliang Jin ${ }^{12}$, Kate Kim ${ }^{12}$, Victor L. Lam ${ }^{12}$, Fei $\mathrm{Li}^{12}$, Junrui Li ${ }^{12}$, Yen-Li Li ${ }^{12}$, Yang Li $^{12}$, Xi Liu' ${ }^{12}$, Megan Lo ${ }^{12}$, Kyle E. Lopez ${ }^{12}$, Arthur A. Melo' ${ }^{12}$, Frank R. Moss III $^{12}$, Phuong Nguyen ${ }^{12}$, Joana Paulino ${ }^{12}$, Komal Ishwar Pawar ${ }^{12}$, Jessica K. Peters ${ }^{12}$, Thomas H. Pospiech Jr. ${ }^{12}$, Maliheh Safari $^{12}$, Smriti Sangwan ${ }^{12}$, Kaitlin Schaefer ${ }^{12}$, Paul V. Thomas ${ }^{12}$, Aye C. Thwin ${ }^{12}$, Raphael Trenker ${ }^{12}$, Eric Tse $^{12}$, Tsz Kin Martin Tsui ${ }^{12}$, Feng Wang ${ }^{12}$, Natalie Whitis ${ }^{12}$, Zanlin Yu ${ }^{12}$, Kaihua Zhang ${ }^{12}$, Yang Zhang ${ }^{12}$,

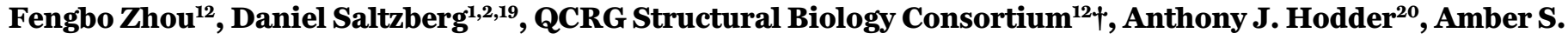
Shun-Shion $^{20}$, Daniel M. Williams ${ }^{20}$, Kris M. White ${ }^{21,22}$, Romel Rosales ${ }^{21,22}$, Thomas Kehrer ${ }^{21,22}$, Lisa Miorin ${ }^{21,22}$, Elena Moreno ${ }^{21,22}$, Arvind H. Patel ${ }^{23}$, Suzannah Rihn ${ }^{23}$, Mir M. Khalid ${ }^{4}$, Albert Vallejo-Gracia ${ }^{4}$, Parinaz Fozouni $^{4,5,7}$, Camille R. Simoneau ${ }^{4,7}$, Theodore L. Roth ${ }^{5,6,7}$, David Wu ${ }^{5,7}$, Mohd Anisul Karim ${ }^{24,25}$, Maya Ghoussaini $^{24,25}$, Ian Dunham ${ }^{16,25}$, Francesco Berardi ${ }^{26}$, Sebastian Weigang ${ }^{27}$, Maxime Chazal ${ }^{28}$, Jisoo Park ${ }^{29}$, James Logue $^{30}$, Marisa McGrath ${ }^{30}$, Stuart Weston ${ }^{30}$, Robert Haupt ${ }^{30}$, C. James Hastie ${ }^{31}$, Matthew Elliott ${ }^{31}$, Fiona Brown $^{31}$, Kerry A. Burness ${ }^{31}$, Elaine Reid ${ }^{31}$, Mark Dorward ${ }^{31}$, Clare Johnson ${ }^{31}$, Stuart G. Wilkinson ${ }^{31}$, Anna Geyer $^{31}$, Daniel M. Giesel ${ }^{31}$, Carla Baillie ${ }^{31}$, Samantha Raggett ${ }^{31}$, Hannah Leech ${ }^{31}$, Rachel Toth ${ }^{31}$, Nicola Goodman $^{31}$, Kathleen C. Keough ${ }^{4}$, Abigail L. Lind ${ }^{4}$, Zoonomia Consortium $\star$, Reyna J. Klesh ${ }^{32}$, Kafi R. Hemphill $^{33}$, Jared Carlson-Stevermer ${ }^{34}$, Jennifer Oki ${ }^{34}$, Kevin Holden ${ }^{34}$, Travis Maures ${ }^{34}$, Katherine S. Pollard $^{4,35,36}$, Andrej Sali ${ }^{1,2,14,19}$, David A. Agard ${ }^{1,2,12,37}$, Yifan Cheng ${ }^{1,2,12,15,37}$, James S. Fraser ${ }^{1,2,12,19}$, Adam Frost $^{1,2,12,37}$, Natalia Jura ${ }^{1,2,3,12,38}$, Tanja Kortemme ${ }^{1,2,12,19,39}$, Aashish Manglik, ${ }^{1,2,12,14}$, Daniel R. Southworth, ${ }^{1,12,37}$, Robert M. Stroud ${ }^{1,2,12,37}$, Dario R. Alessi' ${ }^{31}$, Paul Davies ${ }^{31}$, Matthew B. Frieman ${ }^{30}$, Trey Ideker ${ }^{29,40}$, Carmen Abate $^{26}$, Nolwenn Jouvenet ${ }^{27,28}$, Georg Kochs ${ }^{27}$, Brian Shoichet ${ }^{1,2,14}$, Melanie Ott ${ }^{4,41}$, Massimo Palmarini ${ }^{23}$, Kevan M. Shokat ${ }^{1,2,3,15}$, Adolfo García-Sastre ${ }^{21,22,42,43} \S$, Jeremy A. Rassen ${ }^{11} \S$, Robert Grosse ${ }^{9,44} \S$, Oren S. Rosenberg ${ }^{1,2,12,36,37,41} \S$, Kliment A. Verba ${ }^{1,2,12,14}$ §, Christopher F. Basler ${ }^{10} \S$, Marco Vignuzzi $^{8} \S$, Andrew A. Peden $^{20} \S$, Pedro Beltrao ${ }^{16} \S$, Nevan J. Krogan ${ }^{1,2,3,4} \S$

${ }^{1}$ QBI COVID-19 Research Group (QCRG), San Francisco, CA 94158, USA. ${ }^{2}$ Quantitative Biosciences Institute (QBI), University of California, San Francisco, CA 94158, USA. ${ }^{3}$ Department of Cellular and Molecular Pharmacology, University of California, San Francisco, CA 94158, USA. 4 J. David Gladstone Institutes, San Francisco, CA 94158, USA. ${ }^{5}$ Medical Scientist Training Program, University of California, San Francisco, CA 94143, USA. ${ }^{D}$ Department of Microbiology and Immunology, University of California, San Francisco, CA 94143, USA. ${ }^{7}$ Biomedical Sciences Graduate Program, University of California, San Francisco, CA 94143, USA. ${ }^{8}$ Viral Populations and Pathogenesis Unit, CNRS UMR 3569, Institut Pasteur, 75724, Paris, cedex 15, France. ${ }^{9}$ Institute for Clinical and Experimental Pharmacology and Toxicology I, University of Freiburg, 79104 Freiburg, Germany. ${ }^{10}$ Center for Microbial Pathogenesis, Institute for Biomedical Sciences, Georgia State University, Atlanta, GA 30303, USA. ${ }^{11}$ Aetion, Inc. 5 Penn Plaza, 7th Floor New York, NY 10001, USA. ${ }^{12}$ Quantitative Biosciences Institute (QBI) Coronavirus Research Group Structural Biology Consortium, University of California, San Francisco, CA 94158, USA. ${ }^{13}$ Department of Computational Medicine and Bioinformatics, University of Michigan, Ann Arbor, MI 48109, USA. ${ }^{14}$ Department of Pharmaceutical Chemistry, University of California, San Francisco, CA 94158, USA. ${ }^{15}$ Howard Hughes Medical Institute. ${ }^{16}$ European Molecular Biology Laboratory, European Bioinformatics Institute (EMBL-EBI), Wellcome Genome Campus, Hinxton, Cambridgeshire CB10 1SD, UK. ${ }^{17}$ Division of Basic Sciences, Fred Hutchinson Cancer Research Center, Seattle, WA 98109, USA. ${ }^{18}$ Beam Therapeutics, Cambridge, MA 02139, USA. ${ }^{19}$ Department of Bioengineering and Therapeutic Sciences, University of California, San Francisco, CA 94158, USA. ${ }^{20}$ Department of Biomedical Science, Centre for Membrane Interactions and Dynamics, University of Sheffield, Firth Court, Sheffield S10 2TN, UK. 


\begin{abstract}
${ }^{21}$ Department of Microbiology, Icahn School of Medicine at Mount Sinai, New York, NY 10029, USA. ${ }^{22}$ Global Health and Emerging Pathogens Institute, Icahn School of Medicine at Mount Sinai, New York, NY 10029, USA. ${ }^{23}$ MRC-University of Glasgow Centre for Virus Research, 464 Bearsden Road, Glasgow G61 1QH, Scotland, UK. ${ }^{24}$ Wellcome Trust Sanger Institute, Wellcome Genome Campus, Hinxton, Cambridgeshire CB10 1SA, UK. ${ }^{25}$ Open Targets, Wellcome Genome Campus, Hinxton, Cambridgeshire CB10 1SD, UK. ${ }^{26}$ Dipartimento di Farmacia-Scienze del Farmaco, Università degli Studi di Bari 'ALDO MORO', Via Orabona, 4 70125, Bari, Italy. ${ }^{27}$ Institute of Virology, Medical Center-University of Freiburg, 79104 Freiburg, Germany. ${ }^{28}$ Département de Virologie, CNRS UMR 3569, Institut Pasteur, Paris 75015, France. ${ }^{29}$ Department of Medicine, University of California, San Diego, CA 92093, USA. ${ }^{30}$ Department of Microbiology and Immunology, University of Maryland School of Medicine, Baltimore, MD 21201, USA. ${ }^{31}$ MRC Protein Phosphorylation and Ubiquitylation Unit, College of Life Sciences, University of Dundee, Dundee DD15EH, UK. ${ }^{32} \mathrm{HealthVerity,}$ Philadelphia, PA 19103, USA. ${ }^{33}$ Department of Neurology, University of California, San Francisco, CA 94143, USA. ${ }^{34}$ Synthego Corporation, Redwood City, CA 94063, USA. ${ }^{35}$ Department of Epidemiology \& Biostatistics, University of California, San Francisco, CA 94158, USA. ${ }^{36}$ Chan-Zuckerberg Biohub, San Francisco, CA 94158 , USA. ${ }^{37}$ Department of Biochemistry and Biophysics, University of California, San Francisco, CA 94158, USA. ${ }^{38}$ Cardiovascular Research Institute, University of California, San Francisco, CA 94158, USA. ${ }^{39}$ The UC Berkeley-UCSF Graduate Program in Bioengineering, University of California, San Francisco, CA 94158, USA. ${ }^{40}$ Department to Bioengineering, University of California, San Diego, CA 92093, USA. ${ }^{41}$ Department of Medicine, University of California, San Francisco, CA 94143, USA. ${ }^{42}$ Department of Medicine, Division of Infectious Diseases, Icahn School of Medicine at Mount Sinai, New York, NY 10029, USA. ${ }^{43 T h e}$ Tisch Cancer Institute, Icahn School of Medicine at Mount Sinai, New York, NY 10029, USA. ${ }^{44}$ Centre for Integrative Biological Signaling Studies (CIBSS), 79104 Freiburg, Germany.

* These authors contributed equally to this work. $†$ The QCRG Structural Biology Consortium collaborators and their affiliations are listed in the supplementary materials. $\ddagger$ The Zoonomia Consortium collaborators and their affiliations are listed in the supplementary materials.
\end{abstract}

§Corresponding author. Email: nevan.krogan@ucsf.edu (N.J.K.); pbeltrao@ebi.ac.uk (P.B.); marco.vignuzzi@pasteur.fr (M.V.); cbasler@gsu.edu (C.F.B.); verba@msg.ucsf.edu (K.A.V.); oren.rosenberg@ucsf.edu (O.S.R.); a.peden@sheffield.ac.uk (A.A.P.); robert.grosse@pharmakol.uni-freiburg.de (R.G.); jeremy.rassen@aetion.com (J.A.R.); Adolfo.Garcia-Sastre@mssm.edu (A.G.-S.)

The COVID-19 (Coronavirus disease-2019) pandemic, caused by the SARS-CoV-2 coronavirus, is a significant threat to public health and the global economy. SARS-CoV-2 is closely related to the more lethal but less transmissible coronaviruses SARS-CoV-1 and MERS-CoV. Here, we have carried out comparative viral-human protein-protein interaction and viral protein localization analysis for all three viruses. Subsequent functional genetic screening identified host factors that functionally impinge on coronavirus proliferation, including Tom70, a mitochondrial chaperone protein that interacts with both SARS-CoV-1 and SARS-CoV-2 Orf9b, an interaction we structurally characterized using cryo-EM. Combining geneticallyvalidated host factors with both COVID-19 patient genetic data and medical billing records identified important molecular mechanisms and potential drug treatments that merit further molecular and clinical study.

In the past two decades, three deadly human respiratory syndromes associated with coronavirus (CoV) infections have emerged: Severe Acute Respiratory Syndrome (SARS) in 2002, Middle East Respiratory Syndrome (MERS) in 2012, and Coronavirus Disease 2019 (COVID-19) in 2019. These three diseases are caused by the zoonotic CoVs SARS-CoV-1, MERS-CoV, and SARS-CoV-2 (1), respectively. Before their emergence, human CoVs were associated with usually mild respiratory illness. To date, SARS-CoV-2 has sickened millions and killed over one million worldwide. This unprecedented challenge has prompted widespread efforts to develop new vaccine and antiviral strategies, including repurposed therapeutics, which offer the potential for treatments with known safety profiles and short development timelines. The successful repurposing of the antiviral nucleoside analog Remdesivir (2), as well as the host-directed anti-inflammatory steroid dexamethasone (3), provide clear proof that existing compounds can be crucial tools in the fight against COVID-19. Despite these promising examples, there is still no curative treatment for COVID-19. In addition, as with any virus, the search for effective antiviral strategies could be complicated over time by the continued evolution of SARS-CoV-2 and possible resulting drug resistance (4).

Current endeavors are appropriately focused on SARSCoV-2 due to the severity and urgency of the ongoing pandemic. However, the frequency with which other highly virulent $\mathrm{CoV}$ strains have emerged highlights an additional need to identify promising targets for broad CoV inhibitors with high barriers to resistance mutations and potential for rapid deployment against future emerging strains. While traditional antivirals target viral enzymes that are often subject to mutation and thus the development of drug resistance, targeting the host proteins required for viral replication is a strategy that can avoid resistance and lead to therapeutics with the potential for broad-spectrum activity as families of viruses often exploit common cellular pathways and processes.

Here, we identified shared biology and potential drug targets among the three highly pathogenic human CoV strains. We expanded upon our recently published map of virus-host protein interactions for SARS-CoV-2 (5) and mapped the full interactome of SARS-CoV-1 and MERS-CoV. We investigated the localization of viral proteins across strains, and quantitatively compared the virus-human interactions for each virus. Using functional genetics and structural analysis of selected host-dependency factors, we identified drug targets and also performed real-world analysis on clinical data from COVID19 patient outcomes.

\section{A cross-coronavirus study of protein function}

A central goal of this study is to understand, from a systems level, the conservation of target proteins and cellular processes between SARS-CoV-2, SARS-CoV-1 and MERS-CoV, and thereby identify shared vulnerabilities that can be 
targeted with antiviral therapeutics. All three strains encode four homologous structural proteins (E, M, N, S) and 16 nonstructural proteins (Nsps). The latter are proteolytically cleaved from a polyprotein precursor that is expressed from one large open reading frame (Orf), Orflab (Fig. 1A). Additionally, coronaviruses contain a variable number of accessory factors encoded by Orfs. While the genome organization and sequence of Orflab is mainly conserved between the three viruses under study, it diverges significantly in the region encoding the accessory factors, especially between MERS-CoV and the two SARS coronaviruses (Fig. 1, A to D, and table S1). These differences in conservation of genes and genome organization are linked to differences in host targeting systems that we have studied through large scale protein localization and interaction profiling (Fig. 1E). Building on our earlier work on the interactome of SARS-CoV-2 (5), we identified the host factors physically interacting with each SARS-CoV-1 and MERS-CoV viral protein. To this end, structural proteins, mature Nsps and predicted Orf proteins were codon optimized, 2xStrep tagged and cloned into a mammalian expression vector (figs. S1 and S2; see below and Methods section). Each protein construct was transfected into HEK293T cells, affinity purified, and high-confidence interactors were identified by mass spectrometry and scored using SAINTexpress and MiST scoring algorithms $(6,7)$ (table S2 and figs. S3 to S6). In addition, we performed mass spectrometry analysis on SARS-CoV-2 Nsp16, which was not analyzed in our earlier work (5) (table S2 and fig. S7). In all, we now report 389 high-confidence interactors for SARS-CoV-2, 366 interactions for SARS-CoV-1, and 296 interactions for MERS-CoV (table S2).

\section{Conserved coronavirus proteins often retain the same cellular localization}

As protein localization can provide important information regarding function, we assessed the cellular localization of individually expressed coronavirus proteins in addition to mapping their interactions (Fig. 2A and Methods). Immunofluorescence localization analysis of all 2xStrep-tagged SARSCoV-2, SARS-CoV-1, and MERS-CoV proteins highlights similar patterns of localization for the vast majority of shared protein homologs in HeLaM cells (Fig. 2B), supporting the hypothesis that conserved proteins share functional similarities. A notable exception is Nsp13, which appears to localize to the cytoplasm for SARS-CoV-2 and SARS-CoV-1; but to the mitochondria for MERS-CoV (Fig. 2B, figs. S8 to S13, and table S3). To assess the localization of SARS-CoV-2 proteins in the context of infected cells, we raised antibodies against 20 of them and validated them with the individually-expressed 2xStrep-tagged proteins (fig. S14). Using the 14 antibodies with confirmed specificity, we observed that localization of viral proteins in infected Caco-2 cells sometimes differed from their localization when expressed individually (Fig. 2B, fig. S15, and table S3). This likely results from recruitment of viral proteins and complexes into replication compartments, as well as remodeling of the secretory pathway during viral infection. Such differences could also be due to miss-localization caused by protein tagging. For example, the localization of expressed Orf7B does not match the known SARS-CoV-1 Golgi localization seen in the infection state. For proteins such as Nsp1 and Orf3a, which are not known to be involved in viral replication, their localization is consistent both when expressed individually and in the context of viral infection (Fig. 2, C and D). We have compared the localization of the expressed viral proteins with the localization of their interaction partners using a cellular compartment Gene Ontology enrichment analysis (fig. S16). Several examples exist where the localization of the viral protein is in agreement with the localization of the interaction partners, including enrichment of the Nuclear Pore for Nsp9 interactors and ER enrichment for interactions with Orf8.

Our localization studies suggest that most orthologous proteins have the same localization across the viruses (Fig. 2B). Moreover, small changes in localization, as observed for some viral proteins across strains, do not coincide with strong changes in viral-host protein interactions (Fig. 2E). Overall, these results suggest that changes in protein localization, as measured by expressed tagged proteins, are not common and therefore they are unlikely to be a major source of differences in host targeting mechanisms.

\section{Comparison of host targeted processes identifies con- served mechanisms with divergent implementations}

To study the conservation of targeted host factors and processes, we first used a clustering approach (Methods) to compare the overlap in protein interactions for the three viruses (Fig. 3A). We defined 7 clusters of viral-host interactions corresponding to those that are specific to each or shared among the viruses. The largest pairwise overlap was observed between SARS-CoV-1 and SARS-CoV-2 (Fig. 3A), as expected from their closer evolutionary relationship. A functional enrichment analysis (Fig. 3B and table S4) highlighted host processes that are targeted through interactions conserved across all three viruses including ribosome biogenesis and regulation of RNA metabolism. Conserved interactions between SARS-CoV-1 and SARS-CoV-2, but not MERS-CoV, were enriched in endosomal and Golgi vesicle transport (Fig. 3B). Despite the small fraction (7.1\%) of interactions conserved between SARS-CoV-1 and MERS-CoV, but not SARS-CoV-2, these were strongly enriched in translation initiation and myosin complex proteins (Fig. 3B).

We next asked if the conserved interactions were specific for certain viral proteins (Fig. 3C), and found that some proteins (M, N, Nsp7/8/13) showed a disproportionately high 
fraction of shared interactions conserved across the three viruses. This suggests that the processes targeted by these proteins may be more essential and more likely to be required for other emerging coronaviruses. Such differences in conservation of interactions should be encoded, to some extent, in the degree of sequence differences. Comparing pairs of homologous proteins shared between SARS-CoV-2 and SARSCoV-1 or MERS-CoV, we observed a significant correlation between sequence conservation and protein-protein interaction (PPI) similarity (calculated as Jaccard index) (Fig. 3D, r = 0.58 , p-value $=0.0001$ ). This shows that the evolution of protein sequences strongly determines the divergence in the host interactors.

While studying the function of host proteins interacting with each virus, we noted that some shared cellular processes were targeted by different interactions across the viruses. To study this in more detail, we identified the cellular processes significantly enriched in the interactomes of all three viruses (fig. S17A and table S4) and ranked them by the degree of overlapping proteins (Fig. 3E). This identified proteins related to the nuclear envelope, proteasomal catabolism, cellular response to heat and regulation of intracellular protein transport as biological functions that are hijacked by these viruses through different human proteins. Additionally, we found that up to $51 \%$ of protein interactions with a conserved human target occurred via a different (non-orthologous) viral protein (Fig. 3F) and, in some cases, the overlap of interactions for two non-orthologous virus baits was greater than that for the orthologous pair (Fig. 3G and fig. S17, B and C). For example, several interacting proteins of SARS-CoV-2 Nsp8 are also targeted by MERS-CoV Orf4a, and interactions of MERS-CoV Orf5 share interactors with SARS-CoV-2 Orf3a (Fig. 3G). In the case of Nsp8, we found some degree of structural homology between the C-terminal region of it and a predicted structural model of Orf4a (Methods and fig. S17D), indicative of a possible common interaction mechanism.

In summary, we find that sequence differences determine the degree of changes in viral-host interactions, and that often the same cellular process can be targeted by different viral or host proteins. These results suggest a degree of plasticity in the way these viruses can control a given biological process in the host cell.

\section{Quantitative differential interaction scoring (DIS) iden- tifies interactions conserved between coronaviruses}

The identification of virus-host interactions conserved across pathogenic coronaviruses provides the opportunity to reveal host targets that may remain essential for these and other emerging coronaviruses. For a quantitative comparison of each virus-human interaction from viral baits shared by all three viruses, we developed a differential interaction score (DIS). DIS is calculated between any pair of viruses and is defined as the difference between the interaction scores $(K)$ from each virus (Fig. 4A, table S5, and Methods). This kind of comparative analysis is beneficial as it permits the recovery of conserved interactions that may fall just below strict cutoffs. For each comparison, DIS was calculated for interactions residing in certain clusters as defined in the previous analysis (see Fig. 3A). For example, for the SARS-CoV-2 to MERS-CoV comparison, a DIS was computed for interactions residing in all clusters except cluster 3 , where interactions are either not found or scores were very low for both SARS-CoV2 and MERS-CoV. A DIS of 0 indicates that the interaction is confidently shared between the two viruses being compared, while a DIS of +1 or -1 indicates that the host protein interaction is specific for the virus listed first or second, respectively.

In agreement with our previous results (Fig. 3A), DIS scores for the comparison between SARS-CoV-2 and SARSCoV-1 are enriched near zero, indicating a high number of shared interactions (Fig. 4B, yellow). On the other hand, comparing interactions from either SARS-CoV-1 or SARS-CoV-2 with MERS-CoV resulted in DIS values closer to \pm 1 , indicating a higher divergence (Fig. 4B, blue and green). The breakdown of DIS by homologous viral proteins reveals high similarity of interactions for proteins N, Nsp8, Nsp7, and Nsp13 (Fig. 4C), reinforcing the observations made by overlapping thresholded interactions (Fig. 3, C and D). As the greatest dissimilarity was observed between the SARS coronaviruses and MERS-CoV, we computed a fourth DIS (SARS-MERS) by averaging $K$ from SARS-CoV-1 and SARS-CoV-2 prior to calculating the difference with MERS-CoV (Fig. 4, B and C, purple). We next created a network visualization of the SARS-MERS comparison (Fig. 4D), permitting an appreciation of SARSspecific (red; DIS near +1 ) versus MERS-specific (blue; DIS near -1) interactions, as well as those conserved between all three coronavirus species (black; DIS near zero). SARSspecific interactions include: DNA polymerase $\alpha$ interacting with Nsp1; stress granule regulators interacting with $\mathrm{N}$ protein; TLE transcription factors interacting with Nsp13; and AP2 clathrin interacting with Nsp10. Notable MERS-CoVspecific interactions include: mTOR and Stat3 interacting with Nsp1; DNA damage response components p53 (TP53), MRE11, RAD50, and UBR5 interacting with Nsp14; and the activating signal cointegrator 1 (ASC-1) complex interacting with Nsp2. Interactions shared between all three coronaviruses include: casein kinase II and RNA processing regulators interacting with $\mathrm{N}$ protein; IMP dehydrogenase 2 (IMPDH2) interacting with Nsp14; centrosome, protein kinase A, and TBK1 interacting with Nsp13; and the signal recognition particle, 7SK snRNP, exosome, and ribosome biogenesis components interacting with Nsp8 (Fig. 4D). 


\section{Cell-based genetic screens identify SARS-CoV-2 host de- pendency factors}

To identify host factors that are critical for infection and therefore potential targets for host-directed therapies, we performed genetic perturbations of 332 human proteins, 331 previously identified to interact with SARS-CoV-2 proteins (5) plus ACE2, and observed their effect on infectivity. To ensure a broad coverage of potential hits, we carried out two screens in different cell lines, investigating the effects on infection: siRNA knockdowns in A549 cells stably expressing ACE2 (A549-ACE2) (Fig. 5A) and CRISPR-based knockouts in Caco2 cells (Fig. 5B). ACE2 was included as positive control in both screens as were non-targeting siRNAs or non-targeted Caco2 cells as negative controls. After SARS-CoV-2 infection, effects on virus infectivity were quantified by RT-qPCR on cell supernatants (siRNA) or by titrating virus-containing supernatants on Vero E6 cells (CRISPR) (see Methods for details). Cells were monitored for viability and knockdown or editing efficiency was determined as described (Methods and fig. S18). This revealed that $93 \%$ of the genes were knocked down at least $50 \%$ in the A549-ACE2 screen, and $95 \%$ of the knockdowns exhibited less than a $20 \%$ decrease in viability. In the Caco-2 assay, we observed an editing efficiency of at least $80 \%$ for $89 \%$ of the genes tested (Methods and fig. S18). Of the 332 human SARS-CoV-2 interactors, the final A549-ACE2 dataset includes 331 gene knockdowns and the Caco-2 dataset includes 286 gene knockouts, with the difference mainly due to removal of essential genes (Methods). The readouts from both assays were then separately normalized using robust Zscores (Methods), with negative and positive Z-scores indicating proviral dependency factors (perturbation = decreased infectivity) and antiviral host factors with restrictive activity (perturbation $=$ increased infectivity), respectively. As expected, negative controls resulted in neutral Z-scores (Fig. 5, $\mathrm{C}$ and D, and tables S6 and S7). Similarly, perturbations of the positive control ACE2 resulted in strongly negative Zscores in both assays (Fig. 5, C and D). Overall, the Z-scores did not exhibit any trends related to viability, knockdown efficiency, or editing efficiency (fig. S18). With a cutoff of $|Z|>$ 2 to highlight genes that notably affect SARS-CoV-2 infectivity when perturbed, 31 and 40 dependency factors $(Z<-2)$ and 3 and 4 factors with restrictive activity $(Z>2)$ were identified in A549-ACE2 and Caco-2 cells, respectively (Fig. 5E). Of particular interest are the host dependency factors for SARS-CoV-2 infection, which represent potential targets for drug development and repurposing. For example, non-opioid receptor sigma 1 (sigma-1, encoded by SIGMAR1) was identified as a functional host-dependency factor in both cell systems in agreement with our previous report of antiviral activity for sigma receptor ligands (5). To provide a contextual view of the genetics results, we generated a network that integrates the hits from both cell lines and the PPIs of their encoded proteins with SARS-CoV-2, SARS-CoV-1 and MERS$\mathrm{CoV}$ proteins (Fig. $5 \mathrm{~F}$ ). Interestingly, we observed an enrichment of genetic hits that encode proteins interacting with viral Nsp7, which has a high degree of interactions shared across all the three viruses (Fig. 3C). Prostaglandin E synthase 2 (encoded by PTGES2), for example, is a functional interactor of Nsp7 from SARS-CoV-1, SARS-CoV-2 and MERS$\mathrm{CoV}$. Other dependency factors were specific to SARS-CoV-2, including interleukin-17 receptor A (ILI7RA), which interacts with SARS-CoV-2 Orf8. We also identify dependency factors that are shared interactors between SARS-CoV-1 and SARSCoV-2, such as the aforementioned sigma receptor 1 (SIGMAR1) which interacts with Nsp6, and the mitochondrial import receptor subunit Tom70 (TOMM70) which interacts with Orf9b. We will use these interactions to validate virushost interactions (Orf8-IL17RA and Orf9b-Tom70), connect our systems biology data to evidence for clinical impact of the host factors we identified (IL17RA), and analyze outcomes of COVID-19 patients treated with putative host-directed drugs against PGES-2 and sigma receptor 1.

\section{SARS Orf9b Interacts with Tom70}

Orf9b of SARS-CoV-1 and SARS-CoV-2 was found to be localized to mitochondria upon overexpression as well as in SARSCoV-2 infected cells. In line with this, the mitochondrial outer membrane protein Tom70 (encoded by TOMM70) is a highconfidence interactor of Orf9b in both SARS-CoV-1 and SARSCoV-2 interaction maps (Fig. 6A) and may act as a host dependency factor for SARS-CoV-2 (Fig. 6B). Tom70 falls below the scoring threshold as a putative interactor of MERS-CoV Nsp2, a viral protein not associated with mitochondria. (table $\mathrm{S} 2$ ). Tom70 is one of the major import receptors in the TOM complex that recognizes and mediates the translocation of mitochondrial preproteins from the cytosol into the mitochondria in a chaperone dependent manner (8). Additionally, Tom70 is involved in the activation of the mitochondrial antiviral signaling (MAVS) protein which leads to apoptosis upon virus infection $(9,10)$.

To validate the interaction between viral proteins and Tom70, we performed a co-immunoprecipitation experiment in the presence or absence of Strep-tagged Orf9b from SARSCoV-1 and SARS-CoV-2 as well as Strep-tagged Nsp2 from all three CoVs. Endogenous Tom70, but not other translocase proteins of the outer membrane including Tom20, Tom22 and Tom40, co-precipitated only in the presence of Orf9b but not Nsp2 in both HEK293T and A549 cells, confirming our AP-MS data and suggesting that Orf9b specifically interacts with Tom70 (Fig. 6C and fig. S19A). Further, upon co-expression in bacterial cells, we were able to co-purify the Orf9bTom70 protein complex, indicating a stable complex (Fig. $6 \mathrm{D})$. We found SARS-CoV-1 and SARS-CoV-2 Orf9b expressed in HeLaM cells co-localized with Tom70 (Fig. 6E) and 
observed that SARS-CoV-1 or SARS-CoV-2 Orf9b overexpression led to decreases in Tom70 expression (Fig. 6, E and F). Similarly, Orf9b was found to co-localize with Tom70 upon SARS-CoV-2 infection (Fig. 6G). This is in agreement with the known outer mitochondrial membrane localization of Tom70 (11) and Orf9b localization to mitochondria upon overexpression and during SARS-CoV-2 infection (Fig. 2B). We also saw decreases in Tom70 expression during SARS-CoV-2 infection (Fig. 6G) but did not see dramatic changes in expression levels of the mitochondrial protein Tom20 after individual Strep-Orf9b expression or upon SARS-CoV-2 infection (fig. S19, B and C).

\section{CryoEM structure of Orf9b-Tom70 complex reveals Orf9b interacting at the substrate binding site of Tom70}

Tom70, as part of the Tom complex, is involved in recognition of mitochondrial pre-proteins from the cytosol (12). To further understand the molecular details of Orf9b-Tom70 interactions, we obtained a $3 \AA$ cryoEM structure of the Orf9bTom70 complex (Fig. 7A and fig. S20). Interestingly, although purified proteins failed to interact upon attempted in vitro complex reconstitution, they yielded a stable and pure complex when co-expressed in E. coli (Fig. 6D). This may be due to the fact that Orf9b alone purifies as a dimer (as inferred by the apparent molecular weight on size exclusion chromatography) and would need to dissociate to interact with Tom70 based on our structure. Tom70 preferentially binds preproteins with internal hydrophobic targeting sequences (13). It contains an N-terminal transmembrane domain and tetratricopeptide repeat (TPR) motifs in its cytosolic segment. The C-terminal TPR motifs recognize the internal mitochondrial targeting signals (MTS) of preproteins, and the N-terminal TPR clamp domain serves as a docking site for multichaperone complexes that contain preprotein $(14,15)$. Obtained cryoEM density allowed us to build atomic models for residues 109-600 of human Tom70 and residues 39-76 of SARS-CoV-2 Orf9b (Fig. 7A and table S8). Orf9b makes extensive hydrophobic interactions at the pocket on Tom70 that has been implicated in its binding to MTS, with the total buried surface area at the interface being quite extensive, approximately $2000 \mathrm{~A}^{2}$ (Fig. 7B). In addition to the mostly hydrophobic interface, four salt bridges further stabilize the interaction (Fig. 7C). Upon interaction with Orf9b, the interacting helices on Tom70 move inward to tightly wrap around Orf9b as compared to previously crystallized yeast Tom70 homologs (movie S1). No structure for human Tom70 without a substrate has been reported to date and therefore we cannot rule out that the conformational differences are due to differences between homologs. However, it is possible that this conformational change upon substrate binding is conserved across homologs as many of the Tom70 residues interacting with Orf9b are highly conserved, likely indicating residues essential for endogenous MTS substrate recognition.

Surprisingly, although a previously published crystal structure of SARS-CoV-2 Orf9b revealed that it entirely consists of beta sheets (PDB:6Z4U) (16), upon binding Tom70 residues 52-68, Orf9b forms a helix (Fig. 7D). This is consistent with the fact that MTS sequences recognized by Tom70 are usually helical, and analysis with the TargetP MTS prediction server revealed a high probability for this region of Orf9b to possess an MTS (Fig. 7E). This shows structural plasticity in this viral protein where, depending on the binding partner, Orf9b changes between helical and beta strand folds. Furthermore, we had previously identified two infection-driven phosphorylation sites on Orf9b, S50 and S53 (17), which map to the region on Orf9b buried deep in the Tom70 binding pocket (Fig. 7B, yellow). S53 contributes two hydrogen bonds to the interaction with Tom70 in this overall hydrophobic region. Therefore, once phosphorylated, it is likely that the Orf9b-Tom70 interaction is weakened. These residues are surface exposed in the dimeric structure of the Orf9b, which could potentially allow phosphorylation to partition Orf9b between Tom70-bound and dimeric populations.

The two binding sites on Tom70-the substrate binding site and the TPR domain that recognizes Hsp70/Hsp90-are known to be conformationally coupled $(17,18)$. Tom70's interaction with a C-terminal EEVD motif of Hsp90 via the TPR domain is key for its function in the interferon pathway, and induction of apoptosis upon virus infection $(10,19)$. Whether Orf9b, by binding to the substrate recognition site of Tom70, allosterically inhibits Tom70's interaction with Hsp90 at the TPR domain remains to be investigated but interestingly, we see in our structure that R192, a key residue in the interaction with Hsp70/Hsp90, is moved out of position to interact with the EEVD sequence, suggesting that Orf9b may modulate interferon and apoptosis signaling via Tom70 (fig. S21). Alternatively, Tom70 has been described as an essential import receptor for PTEN induced kinase 1 (PINK1) and therefore loss of mitochondrial import efficiency as a result of Orf9b binding to Tom70 substrate binding pocket may induce mitophagy.

\section{Implications of the Orf8-IL17RA interaction for COVID-19}

As described above, we found that IL-17 receptor A (IL17RA) physically interacts with Orf8 from SARS-CoV-2, but not SARS-CoV-1 or MERS-CoV (Fig. 5D, table S2, and Fig. 8A). Interestingly, several recent studies have identified high IL-17 levels or aberrant IL-17 signaling as a correlate of severe COVID-19 (20-23). We demonstrated the physical interaction of SARS-CoV-2 Orf8 with IL17RA occurs with or without IL17A treatment, suggesting that signaling through the receptor does not disrupt the interaction with Orf8 (Fig. 8B). Furthermore, knockdown of IL17RA led to a significant decrease in 
SARS-CoV-2 viral replication in A549-ACE2 cells (Fig. 8C). These data suggest that the Orf8-IL17RA interaction modulates systemic IL-17 signaling.

One manner in which this signaling is regulated is through the release of the extracellular domain of the receptor as soluble IL17RA (sIL17RA), which acts as a decoy in circulation by soaking up IL-17A and inhibiting IL-17 signaling (21). Production of SIL17RA has been demonstrated by alternative splicing in cultured cells (22), but the mechanism by which IL17RA is shed in vivo remains unclear (23). ADAM family proteases are known to mediate the release of other interleukin receptors into their soluble form (24). We found that SARS-CoV-2 Orf8 physically interacted with both ADAM9 and ADAMTS1 in our previous study (5). We find that knockdown of ADAM9, like that of IL17RA, leads to significant decreases in SARS-CoV-2 replication in A549-ACE2 cells (Fig. 5D and table S2).

In order to test the in vivo relevance of SIL17RA in modulating SARS-CoV-2 infection, we leveraged a genome-wide association study (GWAS) which identified 14 single nucleotide polymorphisms (SNPs) near the IL17RA gene that causally regulate sIL17RA plasma levels (25). We then used generalized summary-based Mendelian randomization (GSMR) (25, 26 ) on the curated GWAS datasets of the COVID-19 Host Genetics Initiative (COVID-HGI) (27) and observed that genotypes that predicted higher sIL17RA plasma levels were associated with lower risk of COVID-19 when compared to the population (Fig. 8D and table S9), seemingly consistent with our molecular data. Similar results were obtained when comparing only hospitalized COVID-19 patients to the population. However, there was no evidence of association in hospitalized versus non-hospitalized COVID-19 patients. Though the COVID-HGI dataset is underpowered and this observation needs to be replicated in other cohorts, the clinical observations, functional genetics and clinical genetics all suggest that SARS-CoV-2 benefits from modulating IL-17 signaling. One potentially contradictory caveat is that we find high-level IL-17A treatment diminishes SARS-CoV-2 replication in A549-ACE2 cells (fig. S22), however IL-17 is a pleiotropic cytokine and it is likely to play multiple roles during SARS-CoV-2 infection in the context of a competent immune system.

Interestingly, infectious and transmissible SARS-CoV-2 viruses with large deletions of Orf8 have arisen during the pandemic and have been associated with milder disease and lower concentrations of pro-inflammatory cytokines (20). Notably, compared to healthy controls, patients infected with wildtype, but not Orf8-deleted virus, had three-fold elevated plasma levels of IL-17A (20). More work will be needed to understand if and how Orf8 manipulates the IL-17 signaling pathway during the course of SARS-CoV-2 infection.

\section{Investigation of druggable targets identified as interac- tors of multiple coronaviruses}

The identification of druggable host factors provides a rationale for drug repurposing efforts. Given the extent of the current pandemic, real-world data can now be used to study the outcome of COVID-19 patients coincidentally treated with host factor-directed, FDA-approved therapeutics. Using medical billing data, we identified 738,933 patients in the United States with documented SARS-CoV-2 infection (Methods). In this cohort, we probed the use of drugs against targets identified here that were shared across coronavirus strains and found to be functionally relevant in the genetic perturbation screens. In particular, we analyzed outcomes for an inhibitor of prostaglandin E synthase type 2 (PGES-2, encoded by PTGES2) and for potential ligands of sigma non-opioid receptor 1 (sigma-1, encoded by SIGMAR1), and asked whether these patients fared better than carefully-matched patients treated with clinically-similar drugs that do not act on coronavirus host factors.

PGES-2, an interactor of Nsp7 from all three viruses (Fig. 4D), is a dependency factor for SARS-CoV-2 (Fig. 5F). It is inhibited by the FDA-approved prescription nonsteroidal antiinflammatory drug (NSAID) indomethacin. Computational docking of Nsp7 and PGES-2 to predict binding configuration showed that the dominant cluster of models localizes Nsp7 adjacent to the PGES-2-indomethacin binding site (fig. S23). However, indomethacin did not inhibit SARS-CoV-2 in vitro at reasonable antiviral concentrations (fig. S24 and table S10). A previous study also found that similarly high levels of the drug were needed for inhibition of SARS-CoV-1 in vitro, but still showed efficacy for indomethacin against canine coronavirus in vivo (24). This motivated us to observe outcomes in a cohort of outpatients with confirmed SARS-CoV-2 infection who by happenstance initiated a course of indomethacin, as compared to those who initiated the prescription NSAID celecoxib, which lacks anti-PGES-2 activity. We compared the odds of hospitalization by risk-set sampling (RSS) patients treated at the same time and at similar levels of disease severity and then further matching on propensity score (PS) (25) (Fig. 9A and table S11). RSS and PS, combined with a new user, active comparator design that mimics the interventional component of parallel group randomized studies, are established design and analytic techniques that mitigate biases that can arise in observational studies. A complete list of risk factors used for matching, which include demographic data, baseline healthcare utilization, comorbidities and measures of disease severity, are found in table S11.

Among SARS-CoV-2-positive patients, new users of indomethacin in the outpatient setting were less likely than matched new users of celecoxib to require hospitalization or inpatient services (Fig. 9B; Odds Ratio (OR) $=0.33$, 95\% Confidence Interval (CI) 0.03-3.19). The confidence interval of our 
primary analysis included the null value. In sensitivity analyses, neither using the larger, risk-set-sampled cohort nor relaxing our outcome definition to include any hospital visit appreciably changed the interpretation of our findings, but it did narrow the confidence intervals, particularly when both approaches were combined (OR $=0.25,95 \%$ CI $0.08-0.76)$. While it is important to acknowledge that this is a small, noninterventional study, it is nonetheless a powerful example of how molecular insight can rapidly generate testable clinical hypotheses and help prioritize candidates for prospective clinical trials or future drug development.

To create larger patient cohorts, we next grouped drugs that shared activity against the same target, sigma receptors. We previously identified sigma-1 and sigma-2 as drug targets in our SARS-CoV-2-human protein-protein interaction map and multiple potent, non-selective sigma ligands were among the most promising inhibitors of SARS-CoV-2 replication in Vero E6 cells (5). As shown above, knockout and knockdown of SIGMAR1, but not SIGMAR2 (also known as TMEM97), led to robust decreases in SARS-CoV-2 replication (fig. S24 and Fig. $5 \mathrm{~F}$ ), suggesting that sigma-1 may be a key therapeutic target. We analyzed SIGMAR1 sequences across 359 mammals and observed positive selection of several residues within beaked whale, mouse, and ruminant lineages, which may indicate a role in host-pathogen competition (fig. S25). Additionally, the sigma ligand drug amiodarone inhibited replication of SARS-CoV-1 as well as SARS-CoV-2, consistent with the conservation of the Nsp6-sigma-1 interaction across the SARS viruses (fig. S24 and Fig. 4D). We then looked for other FDA-approved drugs with reported nanomolar affinity for sigma receptors or that fit the sigma ligand chemotype (5, $26-33$ ) and selected 13 such therapeutics. We find that all are potent inhibitors of SARS-CoV-2 with $\mathrm{IC}_{50}$ values under 10 $\mu \mathrm{M}$, though it is important to note there is a wide range in sigma receptor affinity with no clear correlation between sigma receptor binding affinity and antiviral activity (fig. S24D). Several clinical drug classes were represented by more than one candidate, including typical antipsychotics and antihistamines. Over-the-counter antihistamines are not well represented in medical billing data and are therefore poor candidates for real-world analysis, but users of typical antipsychotics can be easily identified in our patient cohort. By grouping these individual drug candidates by clinical indication, we were able to build a better-powered comparison.

We constructed a cohort for retrospective analysis on new, inpatient users of antipsychotics. In inpatient settings, typical and atypical antipsychotics are used similarly, most commonly for delirium. We compared the effectiveness of typical antipsychotics, which have sigma activity and antiviral effects (fig. S24E), versus atypical antipsychotics, which are not predicted to bind sigma receptors and do not have antiviral activity (fig. S24F), for treatment of COVID-19 (Fig. 9C).
Observing mechanical ventilation outcomes in inpatient cohorts is a proxy for worsening of severe illness, rather than the progression from mild disease signified by the hospitalization of indomethacin-exposed outpatients above. We again employed RSS plus PS to build a robust, directly comparable cohort of inpatients (table S11). In our primary analysis, half as many new users of the sigma-ligand typical antipsychotics compared to new users of atypical antipsychotics progressed to the point of requiring mechanical ventilation, demonstrating significantly lower use with an odds ratio (OR) of 0.46 (95\% CI $=0.23-0.93, \mathrm{p}=0.03$, Fig. 9D). As above, we conducted a sensitivity analysis in the RSS-only cohort and observed the same trend $(\mathrm{OR}=0.56,95 \% \mathrm{CI}=0.31-1.02, \mathrm{p}=$ 0.06), emphasizing the primary result of a beneficial effect for typical versus atypical antipsychotics observed in the RSSplus-PS-matched cohort. Although a careful analysis of the relative benefits and risks of typical antipsychotics should be undertaken before considering prospective studies or interventions, these data and analysis demonstrate how molecular information can be translated into real-world implications for the treatment of COVID-19, an approach that can ultimately be applied to other diseases in the future.

\section{Discussion}

In this study, we generated and compared three different coronavirus-human protein-protein interaction maps in an attempt to identify and understand pan-coronavirus molecular mechanisms. The use of a quantitative differential interaction scoring (DIS) approach permitted the identification of virus-specific as well as shared interactions among distinct coronaviruses. We also systematically carried out subcellular localization analysis using tagged viral proteins as well as antibodies targeting specific SARS-CoV-2 proteins. Our results suggest that protein localization can often differ when comparing individually-expressed viral proteins with the localization of the same protein in the context of infection. This can be due to factors such as miss-location driven by tagging, changes in localization due to interaction partners, or cellular compartments that are specific to the infection state. These differences are important caveats of viral-host interaction studies performed by tagged expressed proteins. However, previous studies and the work performed here shows how these data can be very powerful for the identification of host targeted processes and relevant drug targets.

These data were integrated with genetic data where the interactions uncovered with SARS-CoV-2 were perturbed using RNAi and CRISPR in different cellular systems and viral assays, an effort that functionally connected many host factors to infection. One of these, Tom70, which we have shown binds to Orf9b from both SARS-CoV-1 and SARS-CoV-2, is a mitochondrial outer membrane translocase that has been previously shown to be important for mounting an interferon 
response (34). Our functional data, however, shows that Tom70 has at least some role in promoting infection rather than inhibiting it. Using cryoEM, we obtained a $3 \AA$ structure of a region of Orf9b binding to the active site of Tom70. Remarkably, we find that Orf9b is in a drastically different conformation than previously visualized. This offers the possibility that Orf9b may partition between two distinct structural states in the cells, with each possessing a different function and possibly explaining its potential functional pleiotropy. The exact details of functional significance and regulation of the Orf9b-Tom70 interaction await further experimental elucidation. This interaction, however, which is conserved between SARS-CoV-1 and SARS-CoV-2, could have value as a pan-coronavirus therapeutic target.

Finally, we attempted to connect our in vitro molecular data to clinical information available for COVID-19 patients to understand the pathophysiology of COVID-19 and explore new therapeutic avenues. To this end, using GWAS datasets of the COVID-19 Host Genetics Initiative (35), we observed that increased predicted sIL17RA plasma levels were associated with lower risk of COVID-19. Interestingly, we find that IL17RA physically binds to SARS-CoV-2 Orf8 and genetic disruption results in decreased infection. These collective data suggest that future studies should be focused on this pathway as both an indicator and therapeutic target for COVID-19. Furthermore, using medical billing data, we also observed trends in COVID-19 patients on specific drugs indicated by our molecular studies. For example, inpatients prescribed sigma-ligand typical antipsychotics seemingly have better COVID-19 outcomes when compared to users of atypical antipsychotics, which do not bind to sigma-1. We cannot be certain that sigma receptor interaction is the mechanism underpinning this effect, as typical antipsychotics are known to bind to a multitude of cellular targets. Replication in other patient cohorts and further work will be needed to see if there is therapeutic value in these connections, but at the very least we have demonstrated a strategy wherein protein network analyses can be used to make testable predictions from realworld, clinical information.

Overall, we have described an integrative and collaborative approach to study and understand pathogenic coronavirus infection, identifying conserved targeted mechanisms that are likely to be of high relevance for other viruses of this family, some of which have yet to infect humans. We used proteomics, cell biology, virology, genetics, structural biology, biochemistry and clinical and genomic information in an attempt to provide a holistic view of SARS-CoV-2 and other coronaviruses' interactions with infected host cells. We propose that such an integrative and collaborative approach could and should be used to study other infectious agents as well as other disease areas.

\section{Materials and Methods \\ Cells}

HEK293T/17 (HEK293T) cells were procured from the UCSF Cell Culture Facility, and are available through UCSF's Cell and Genome Engineering Core (https://cgec.ucsf.edu/cellculture-and-banking-services). HEK293T cells were cultured in Dulbecco's Modified Eagle's Medium (DMEM) (Corning) supplemented with 10\% Fetal Bovine Serum (FBS) (Gibco, Life Technologies) and 1\% Penicillin-Streptomycin (Corning) and maintained at $37^{\circ} \mathrm{C}$ in a humidified atmosphere of $5 \%$ $\mathrm{CO}_{2}$. STR analysis by the Berkeley Cell Culture Facility on August 8, 2017 authenticates these as HEK293T cells with 94\% probability.

HeLaM cells (RRID: CVCL_R965) were originally obtained from the laboratory of M. S. Robinson (CIMR, University of Cambridge, UK) and routinely tested for mycoplasma contamination. HeLaM cells were grown in DMEM supplemented with $10 \% \mathrm{FBS}, 100 \mathrm{U} / \mathrm{ml}$ penicillin, $100 \mu \mathrm{g} / \mathrm{ml}$ streptomycin and $2 \mathrm{mM}$ glutamine at $37^{\circ} \mathrm{C}$ in a $5 \% \mathrm{CO}_{2}$ humidified incubator.

A549 cells stably expressing ACE2 (A549-ACE2) were a kind gift from Dr. Olivier Schwartz. A549-ACE2 cells were cultured in DMEM supplemented with $10 \% \mathrm{FBS}$, blasticidin (20 $\mu \mathrm{g} / \mathrm{ml}$, Sigma) and maintained at $37^{\circ} \mathrm{C}$ with $5 \% \mathrm{CO}_{2}$. STR analysis by the Berkeley Cell Culture Facility on July 17, 2020 authenticates these as A549 cells with 100\% probability.

Caco-2 cells (ATTC, HTB-37, RRID:CVCL_0025) were cultured in DMEM with GlutaMAX and pyruvate (Gibco, 10569010) and supplemented with 20\% FBS (Gibco, 26140079). For Caco-2 cells utilized in Cas9-RNP knockouts, STR analysis by the Berkeley Cell Culture Facility on April 23, 2020 authenticates these as Caco-2 cells with 100\% probability.

Vero E6 cells were purchased from ATCC and thus authenticated (VERO C1008 [Vero 76, clone E6, Vero E6] (ATCC, CRL-1586). Vero E6 cells tested negative for mycoplasma contamination. Vero E6 cells were cultured in DMEM (Corning) supplemented with 10\% Fetal Bovine Serum (FBS) (Gibco, Life Technologies) and 1\% Penicillin-Streptomycin (Corning) and maintained at $37^{\circ} \mathrm{C}$ in a humidified atmosphere of $5 \%$ $\mathrm{CO}_{2}$.

\section{Microbes}

LOBSTER E. coli Expression Strain: LOBSTR-(BL21(DE3)) Kerafast \# EC1002

\section{Antibodies}

Commercially available primary antibodies used in this study:

rabbit anti-beta-Actin (Cell Signaling Technology \#4967, RRID:AB_330288); mouse anti-beta Tubulin (Sigma-Aldrich \#T8328, RRID:AB_1844090); rabbit anti-BiP (Cell Signaling 
Technology \#3177S, RRID:AB_2119845); mouse anti-EEA1 (BD Biosciences \#610457, RRID:AB_397830, used at 1:200); mouse anti-ERGIC53 (Enzo Life Sciences \#ALX-804-602C100, RRID:AB_2051363, used at 1:200); anti-GM130; rabbit anti-GRP78 BiP (Abcam \#Ab21685, RRID:AB_2119834); rabbit anti-SARS-CoV-Nucleocapsid Protein (Rockland \#200401-A50, RRID:AB_828403); rabbit anti-PDI (Cell Signaling Technology \#3501, RRID:AB_2156433); mouse anti-Strep tag (QIAGEN \#34850, RRID:AB_2810987, used at 1:5000); Mouse anti-strepMAB (IBA Lifesciences \#2-1507-001, used at 1:1000); rabbit anti-Strep-tag II (Abcam \#ab232586); rabbit antiTom20 (Proteintech \#11802-1-AP, RRID:AB_2207530, used at 1:1000); rabbit anti-Tom20 (Cell Signaling Technology \#42406, RRID:AB_2687663); mouse anti-Tom22 (Santa Cruz Biotechnology \#sc-101286, RRID:AB_1130526); rabbit antiTom40 (Santa Cruz Biotechnology \#sc-11414, RRID:AB_793274); mouse anti-Tom70 (Santa Cruz \#sc390545, RRID:AB_2714192, used at 1:500); Rabbit anti-STX5 (Synaptic Systems 110 053, used at 1:500); ActinStaining Kit 647-Phalloidin (Hypernol \#8817-01, used at 1:400)

Commercially available secondary antibodies used in this study:

Alexa Fluor 488 chicken anti-mouse IgG (Invitrogen \#A21200, RRID_AB_2535786, used at 1:400); Alexa Fluor 488 chicken anti-rabbit IgG (Invitrogen \#A21441, RRID_AB_10563745, used at 1:400); Alexa Fluor 568 donkey anti-sheep IgG (Invitrogen \#A21099, RRID_AB_10055702, used at 1:400); Alexa Fluor Plus 488 goat anti-rabbit (ThermoFisher A32731, used at 1:500); Alexa Fluor Plus 594 goat anti-mouse (ThermoFisher A32742, used at 1:500); goat anti-mouse IgG-HRP (BioRad \#170-6516, RRID:AB_11125547, used at 1:20000)

\section{Non-commercial antisera}

Rabbit anti-SARS-CoV-2-NP antiserum was produced by the Garcia-Sastre lab and used at 1:10000; for information on polyclonal sheep antibodies targeting SARS-CoV-2 proteins, see below, table S3 and https://mrcppu-covid.bio/.

\section{Coronavimus annotation and plasmid cloning}

SARS-CoV-1 isolate Tor2 (NC_004718) and MERS-CoV (NC_019843) were downloaded from GenBank and utilized to design 2x-Strep tagged expression constructs of open reading frames (Orfs) and proteolytically mature nonstructural proteins (Nsps) derived from Orflab (with N-terminal methionines and stop codons added as necessary). Protein termini were analyzed for predicted acylation motifs, signal peptides, and transmembrane regions, and either the $\mathrm{N}$ - or $\mathrm{C}$ terminus was chosen for tagging as appropriate. Finally, reading frames were codon optimized and cloned into pLVXEF1alpha-IRES-Puro (Takara/Clontech) including a 5' Kozak motif.

\section{Immunofluorescence Microscopy of Viral Protein Con- structs}

Approximately 60,000 HeLaM cells were seeded onto glass coverslips in a 12-well dish and grown overnight. The cells were transfected using $0.5 \mu \mathrm{g}$ of plasmid DNA and either polyethylenimine (Polysciences) or Fugene HD (Promega; 1 part DNA to 3 parts transfection reagent) and grown for a further 16 hours.

Transfected cells were fixed with $4 \%$ paraformaldehyde (Polysciences) in PBS at room temperature for $15 \mathrm{~min}$. The fixative was removed and quenched using $0.1 \mathrm{M}$ glycine in PBS. The cells were permeabilized using $0.1 \%$ saponin in PBS containing $10 \%$ FBS. The cells were stained with the indicated primary and secondary antibodies for 1 hour at room temperature. The coverslips were mounted onto microscope slides using ProLong Gold antifade reagent (ThermoFisher) and imaged using a UplanApo 60x oil (NA 1.4) immersion objective on a Olympus BX61 motorized wide-field epifluorescence microscope. Images were captured using a Hamamatsu Orca monochrome camera and processed using ImageJ.

To gain insight into the intracellular distribution of each Strep-tagged construct, approximately 100 cells per transfection were manually scored. Each construct was assigned an intracellular distribution in relation to the plasma membrane, endoplasmic reticulum, Golgi, cytoplasm and mitochondria (scored out of 7). In several instances the viral proteins were observed on membranes which did not fit any of the basic categories so were defined as being localized on undefined membranes. Many of the constructs had several localizations so this was also reflected in the scoring. The scoring also took into account the impact of expression level on the localization of the constructs.

\section{Meta Analysis of immunofluorescence data}

We first sorted the data concerning viral protein location for all Strep-tagged viral proteins expressed individually in three heatmaps (one per virus) using a custom $\mathrm{R}$ script ("pheatmap" package). The information concerning protein localization during SARS-CoV-2 infection was added as a square border color code in the first heatmap, to compare the two different localization patterns. In order to compare the predicted versus the experimentally determined locations, for each protein we took the top scoring sequence based localization prediction from DeepLoc (36) if the score was bigger than 1 . When more than one localization can be assigned to the same protein, we took as many top scoring ones as experimentally assigned localizations we had for the same protein. Finally, for each cell compartment, we count the number of experimentally assigned viral proteins and the subset of them predicted to that same compartment as "correct predictions". To compare changes in protein interactions with changes in protein localization (Strep-tagged experiment versus 
sequence-based prediction), we calculated the Jaccard index of prey overlap for each viral protein (SARS-CoV-2 vs. SARSCoV-1 and SARS-CoV-2 vs. MERS-CoV) and plotted them together, for proteins with the same localization and for proteins with different localization.

\section{Generation of polyclonal sheep antibodies targeting SARS-CoV-2 proteins}

Sheep were immunized with individual N-terminal GSTtagged SARS-CoV-2 recombinant proteins or N-terminal MBP-tagged proteins (for SARS-CoV-2 S, S-RBD and Orf7a), followed by up to 5 booster injections four weeks apart from each other. Sheep were subsequently bled and IgGs were affinity purified using the specific recombinant $\mathrm{N}$-terminal maltose binding protein (MBP)-tagged viral proteins. Each antiserum specifically recognized the appropriate native viral protein. Characterisation of each antibody can be found at https://mrcppu-covid.bio/. All antibodies generated can be requested at https://mrcppu-covid.bio/. Also see table S3.

\section{Immunofluorescence Microscopy of Infected Caco-2 cells}

For infection experiments in human colon epithelial Caco-2 cells (ATCC, HTB-37), SARS-CoV-2 isolate Muc-IMB-1, kindly provided by the Bundeswehr Institute of Microbiology, $\mathrm{Mu}$ nich, Germany, was used. SARS-CoV-2 was propagated in Vero E6 cells in DMEM supplemented with 2\% FBS. All work involving live SARS-CoV-2 was performed in the BSL3 facility of the Institute of Virology, University Hospital Freiburg, and was approved according to the German Act of Genetic Engineering by the local authority (Regierungspraesidium Tuebingen, permit UNI.FRK.05.16/05).

Caco-2 human colon epithelial cells seeded on glass coverslips were infected with SARS-CoV-2 (Strain Muc-IMB$1 / 2020$, second passage on Vero E6 cells $\left.\left(2 \times 10^{6} \mathrm{PFU} / \mathrm{ml}\right)\right)$ at an MOI of 0.1. At 24 hours post-infection, cells were washed with PBS and fixed in 4\% paraformaldehyde in PBS for 20 min at room temperature, followed by 5 min of quenching in 0.1 M glycine in PBS at room temperature. Cells were permeabilized and blocked in $0.1 \%$ saponin in PBS supplemented with $10 \%$ fetal calf serum for $45 \mathrm{~min}$ at room temperature and incubated with primary antibodies for 1 hour at room temperature. After washing $15 \mathrm{~min}$ with blocking solution, AF568-labeled donkey-anti-sheep (Invitrogen, \#A21099; 1:400) secondary antibody as well as AF4647-labeled Phalloidin (Hypermol, \#8817-01, 1:400) were applied for 1 hour at room temperature. Subsequent washing was followed by embedding in Diamond Antifade Mountant with DAPI (ThermoFisher, \#P36971). Fluorescence images were generated using a LSM800 confocal laser-scanning microscope (Zeiss) equipped with a 63X, 1.4 NA oil objective and Airyscan detector and the Zen blue software (Zeiss) and processed with Zen blue software and ImageJ/Fiji.

\section{Transfection and cell harvest for immunoprecipitation experiments}

For each affinity purification (SARS-CoV-1 baits, MERS-CoV baits, GFP-2xStrep or empty vector controls), ten million HEK293T cells were transfected with up to $15 \mu \mathrm{g}$ of individual expression constructs using PolyJet transfection reagent (SignaGen Laboratories) at a $1: 3 \mu \mathrm{g}: \mu \mathrm{l}$ ratio of plasmid to transfection reagent based on manufacturer's protocol. After more than 38 hours, cells were dissociated at room temperature using $10 \mathrm{ml}$ PBS without calcium and magnesium (DPBS) with $10 \mathrm{mM}$ EDTA for at least $5 \mathrm{~min}$, pelleted by centrifugation at $200 \mathrm{xg}$, at $4^{\circ} \mathrm{C}$ for $5 \mathrm{~min}$, washed with $10 \mathrm{ml} \mathrm{D-PBS,}$ pelleted once more and frozen on dry ice before storage at $80^{\circ} \mathrm{C}$ for later immunoprecipitation analysis. For each bait, three independent biological replicates were prepared.

Whole cell lysates were resolved on $4 \%-20 \%$ Criterion SDS-PAGE gels (Bio-Rad Laboratories) to assess Strep-tagged protein expression by immunoblotting using mouse antiStrep tag antibody 34850 (QIAGEN) and anti-mouse HRP secondary antibody (BioRad).

\section{Anti-Strep-Tag affinity purification}

Frozen cell pellets were thawed on ice for 15-20 min and suspended in $1 \mathrm{ml}$ Lysis Buffer [IP Buffer (50 mM Tris-HCl, pH 7.4 at $4^{\circ} \mathrm{C}, 150 \mathrm{mM} \mathrm{NaCl}, 1 \mathrm{mM}$ EDTA) supplemented with 0.5\% Nonidet P 40 Substitute (NP-40; Fluka Analytical) and cOmplete mini EDTA-free protease and PhosSTOP phosphatase inhibitor cocktails (Roche)]. Samples were then freezefractured by refreezing on dry ice for $10-20 \mathrm{~min}$, then rethawed and incubated on a tube rotator for $30 \mathrm{~min}$ at $4{ }^{\circ} \mathrm{C}$. Debris was pelleted by centrifugation at $13,000 x g$, at $4^{\circ} \mathrm{C}$ for $15 \mathrm{~min}$. Up to 56 samples were arrayed into a 96-well Deepwell plate for affinity purification on the KingFisher Flex Purification System (Thermo Scientific) as follows: MagStrep "type3" beads (30 $\mu \mathrm{l}$; IBA Lifesciences) were equilibrated twice with $1 \mathrm{ml}$ Wash Buffer (IP Buffer supplemented with $0.05 \% \mathrm{NP}-40$ ) and incubated with $0.95 \mathrm{ml}$ lysate for 2 hours. Beads were washed three times with $1 \mathrm{ml}$ Wash Buffer and then once with $1 \mathrm{ml}$ IP Buffer. Beads were released into $75 \mu l$ Denaturation-Reduction Buffer (2 M urea, 50 mM Tris$\mathrm{HCl} \mathrm{pH} \mathrm{8.0,} 1$ mM DTT) in advance of on-bead digestion. All automated protocol steps were performed at $4^{\circ} \mathrm{C}$ using the slow mix speed and the following mix times: $30 \mathrm{~s}$ for equilibration/wash steps, 2 hours for binding, and $1 \mathrm{~min}$ for final bead release. Three $10 \mathrm{~s}$ bead collection times were used between all steps.

\section{On-bead digestion for affinity purification}

Bead-bound proteins were denatured and reduced at $37^{\circ} \mathrm{C}$ for $30 \mathrm{~min}$, alkylated in the dark with $3 \mathrm{mM}$ iodoacetamide for 
$45 \mathrm{~min}$ at room temperature, and quenched with $3 \mathrm{mM}$ DTT for $10 \mathrm{~min}$. To offset evaporation, $22.5 \mu l 50 \mathrm{mM}$ Tris- $\mathrm{HCl}, \mathrm{pH}$ 8.0 were added prior to trypsin digestion. Proteins were then incubated at $37^{\circ} \mathrm{C}$, initially for 4 hours with $1.5 \mu$ l trypsin $(0.5$ $\mu \mathrm{g} / \mu \mathrm{l}$; Promega) and then another $1-2$ hours with $0.5 \mu \mathrm{l}$ additional trypsin. All steps were performed with constant shaking at 1,100 rpm on a ThermoMixer $\mathrm{C}$ incubator. Resulting peptides were combined with $50 \mu \mathrm{l} 50 \mathrm{mM}$ Tris- $\mathrm{HCl}, \mathrm{pH} 8.0$ used to rinse beads and acidified with trifluoroacetic acid (0.5\% final, $\mathrm{pH}<2.0$ ). Acidified peptides were desalted for MS analysis using a BioPureSPE Mini 96-Well Plate (20 mg PROTO $300 \mathrm{C} 18$; The Nest Group, Inc.) according to standard protocols.

\section{Mass spectrometry operation and peptide search}

Samples were re-suspended in $4 \%$ formic acid, $2 \%$ acetonitrile solution, and separated by a reversed-phase gradient over a nanoflow $\mathrm{C} 18$ column (Dr. Maisch). HPLC buffer A was comprised of $0.1 \%$ formic acid, and HPLC buffer B was comprised of $80 \%$ acetonitrile in $0.1 \%$ formic acid. Peptides were eluted by a linear gradient from 7 to $36 \%$ B over the course of $52 \mathrm{~min}$, after which the column was washed with $95 \% \mathrm{~B}$, and re-equilibrated at $2 \% \mathrm{~B}$. Each sample was directly injected via a Easy-nLC 1200 (Thermo Fisher Scientific) into a Q-Exactive Plus mass spectrometer (Thermo Fisher Scientific) and analyzed with a 75 min acquisition, with all MS1 and MS2 spectra collected in the orbitrap; data were acquired using the Thermo software Xcalibur (4.2.47) and Tune (2.11 QF1 Build 3006). For all acquisitions, QCloud was used to control instrument longitudinal performance during the project (37). All proteomic data was searched against the human proteome (uniprot reviewed sequences downloaded February 28th, 2020), EGFP sequence, and the SARS-CoV or MERS protein sequences using the default settings for MaxQuant (version 1.6.12.0) (38). Detected peptides and proteins were filtered to $1 \%$ false discovery rate in MaxQuant. All MS raw data and search results files have been deposited to the ProteomeXchange Consortium via the PRIDE partner repository with the dataset (identifier PXD PXD021588, Username: reviewer_pxd021588@ebi.ac.uk, password: B5Ho3HES).

\section{High-confidence protein interaction scoring}

Identified proteins were then subjected to protein-protein interaction scoring with both SAINTexpress (version 3.6.3) and MiST (https://github.com/kroganlab/mist) $(6,7)$. We applied a two-step filtering strategy to determine the final list of reported interactors, which relied on two different scoring stringency cut-offs. In the first step, we chose all protein interactions that had a MiST score $\geq 0.7$, a SAINTexpress Bayesian false-discovery rate $(\mathrm{BFDR}) \leq 0.05$ and an average spectral count $\geq 2$. For all proteins that fulfilled these criteria, we extracted information about the stable protein complexes that they participated in from the CORUM (39) database of known protein complexes. In the second step, we then relaxed the stringency and recovered additional interactors that (1) formed complexes with interactors determined in filtering step 1 and (2) fulfilled the following criteria: MiST score $\geq 0.6$, SAINTexpress BFDR $\leq 0.05$ and average spectral counts $\geq 2$. Proteins that fulfilled filtering criteria in either step 1 or step 2 were considered to be high-confidence protein-protein interactions (HC-PPIs).

Using this filtering criteria, nearly all of our baits recovered a number of HC-PPIs in close alignment with previous datasets reporting an average of around 6 PPIs per bait (40). However, for a subset of baits, we observed a much higher number of PPIs that passed these filtering criteria. For these baits, the MiST scoring was instead performed using a larger in-house database of 87 baits that were prepared and processed in an analogous manner to this SARS-CoV-2 dataset. This was done to provide a more comprehensive collection of baits for comparison, to minimize the classification of nonspecifically binding background proteins as HC-PPIs. This was performed for SARS-CoV-1 baits (M, Nsp12, Nsp13, Nsp8, and Orf7b), MERS-CoV baits (Nsp13, Nsp2, and Orf4a), and SARS-CoV-2 Nsp16. SARS-CoV-2 Nsp16 MiST was scored using the in-house database as well as all previous SARS-CoV-2 data (5).

\section{Hierarchical clustering of virus-human protein inter- actions}

Hierarchical clustering was performed on interactions for (1) viral bait proteins shared across all three viruses (LIST) and (2) passed the high-confidence scoring criteria (MiST score $\geq$ 0.6 , SAINTexpress BFDR $\leq 0.05$ and average spectral counts $\geq 2$ ) in at least one virus. We clustered using a new Interaction Score $(K)$, which we defined as the average between the MiST and Saint score for each virus-human interaction. This was done to provide a single score that captured the benefits from each scoring method. Clustering was performed using the ComplexHeatmap package in $\mathrm{R}$, using the "average" clustering method and "euclidean" distance metric. K-means clustering $(\mathrm{k}=7)$ was applied to capture all possible combinations of interaction patterns between viruses.

\section{Gene ontology enrichment analysis on clusters}

Sets of genes found in 7 clusters were tested for enrichment of Gene Ontology (GO) terms, which was performed using the enricher function of clusterProfiler package in $\mathrm{R}$ (41). The GO terms were obtained from the C5 collection of Molecular Signature Database (MSigDBv7.1) and include Biological Process, Cellular Component, and Molecular Function ontologies. Significant GO terms were identified (adjusted pvalue $<0.05)$ and further refined to select non-redundant terms. To select non-redundant gene sets, we first 
constructed a GO term tree based on distances (1 - Jaccard Similarity Coefficients of shared genes) between the significant terms. The GO term tree was cut at a specific level $(\mathrm{h}=$ 0.99 ) to identify clusters of non-redundant gene sets. For results with multiple significant terms belonging to the same cluster, we selected the term with the lowest adjusted p-value.

\section{Sequence similarity analysis}

Protein sequence similarity was assessed by comparing the protein sequences from SARS-CoV-1 and MERS-CoV to SARSCoV-2 for orthologous viral bait proteins. The corresponding protein-protein interaction similarity was represented by a Jaccard index, using the high-confidence interactomes for each virus.

\section{Gene ontology enrichment and PPI similarity analysis} The high-confidence interactors of the three viruses were tested for enrichment of GO terms as described above. We then identified GO terms that are significantly enriched (adjusted p-value $<0.05$ ) in all 3 viruses. For each enriched term, we generated the list of its associated genes and computed the Jaccard Index of pairwise comparisons of 3 viruses.

\section{Orthologous versus non-orthologous interactions anal- ysis}

For a given pair of viruses, we identified all pairs of baits that share interactors and categorized these into "orthologous" and "non-orthologous" groups based on whether the two baits were orthologs or not. We then summed up the total number of shared interactors in each group to calculate the corresponding fractions. This was performed for all pairwise combinations of the three viruses.

\section{Structural modeling and comparison of MERS-CoV Orfta and SARS-CoV-2 Nsp8}

To obtain a sensitive sequence comparison between MERSCoV Orf4a and SARS-CoV-2 Nsp8, we took into consideration their homologs. We first searched for homologs of these proteins in the UniRef30 database using hhblits (1 iteration, Evalue cutoff 1e-3) (42). Subsequently, the resulting alignments were filtered to include only sequences with at least $80 \%$ coverage to the corresponding query sequence, and hidden Markov models (HMMs) were created using hhmake. Finally, the HMMs of Orf4a andNsp8 homologs were locally aligned using hhalign. The structure of Orf4a was predicted de novo using trRosetta (43). To provide greater coverage than that provided by experimental structures, SARS-CoV-2 Nsp8 was modeled using the structure of its SARS-CoV homolog as template (PDB: 2AHM) (44) using SWISS-MODEL (45). To search for local structural similarities between Orf4a and Nsp8, we used Geometricus, a structure embedding tool based on 3D rotation invariant moments (46). This generates so-called shape-mers, analogous to sequence k-mers. The structures were fragmented into overlapping k-mers based on the sequence $(\mathrm{k}=20)$ and into overlapping spheres surrounding each residue (radius=15 $\AA$ ). To ensure that the similarities found between these distinct structures were significant, we used a high resolution of 7 to define the shape-mers. This resulted in the identification of 4 different shape-mers common to Orf4a and Nsp8. We aligned the entire Orf4a structure with residues 96 to 191 of the Nsp8 structure (i.e., after removal of the long N-terminal helix) using the Caretta structural alignment algorithm detailed by (47), using 3D rotation invariant moments (Durairaj et al. 2020) for initial superposition. We optimized parameters to maximize the Caretta score. The resulting alignment used $\mathrm{k}=30$, radius $=16 \AA$, gap open penalty $=0.05$, gap extend penalty $=0.005$, and had an root-mean-square deviation (RMSD) of $7.6 \AA$ across 66 aligning residues.

\section{Differential interaction score (DIS) analysis}

We computed a differential interaction score (DIS) for interactions that (1) originated from viral bait proteins shared across all three viruses and (2) passed the high-confidence scoring criteria (MiST score $\geq 0.6$, SAINTexpress BFDR $\leq 0.05$ and average spectral counts $\geq 2$ ) in at least one virus. We defined the DIS to be the difference between the interaction scores $(K)$ from each virus. DIS near 0 indicates that the interaction is confidently shared between the two viruses being compared, while a DIS near -1 or +1 indicates that the host protein interaction is specific for one virus or the other. We computed a fourth DIS (SARS-MERS) by averaging $K$ from SARS-CoV-1 and SARS-CoV-2 prior to calculating the difference with MERS-CoV. Here, a DIS near +1 indicates SARSspecific interactions (shared between SARS-CoV-1 and SARSCoV-2 but absent in MERS-CoV), a DIS near -1 indicates MERS-specific interactions (present in MERS-CoV and absent or lowly confident in both SARS-CoVs), and a DIS near 0 indicates interactions shared between all three viruses.

For each pairwise virus comparison, as well as the SARSMERS comparison, DIS was defined based on cluster membership of interactions (Fig. 3A). For the SARS2-SARS1 comparison, interactions from every cluster except 5 were used, as those interactions are considered absent from both SARSCoV-2 and SARS-CoV-1. For the SARS2-MERS comparison, interactions from all clusters except 3 were used. For the SARS1-MERS comparison, interactions from all clusters except 6 were used. For the SARS-MERS comparison, only interactions from clusters 2,4 , and 5 were used.

\section{Network generation and visualization}

Protein-protein interaction networks were generated in Cytoscape (48) and subsequently annotated using Adobe Illustrator. Host-host physical interactions, protein complex 
definitions, and biological process groupings were derived from CORUM (39), Gene Ontology (biological process), and manually curated from literature sources. All networks were deposited in NDEx (49).

\section{siRNA library and transfection in A549-ACE2 cells}

An OnTargetPlus siRNA SMARTpool library (Horizon Discovery) was purchased targeting 331 of the 332 human proteins previously identified to bind SARS-CoV-2 (5) (PDE4DIP was not available for purchase and excluded from the assay). This library was arrayed in 96-well format, with each plate also including two non-targeting siRNAs and one siRNA pool targeting ACE2 (table S12). The siRNA library was transfected into A549 cells stably expressing ACE2 (A549-ACE2, kindly provided by Dr. Olivier Schwartz), using Lipofectamine RNAiMAX reagent (Thermo Fisher). Briefly, 6 pmoles of each siRNA pool were mixed with $0.25 \mu$ l RNAiMAX transfection reagent and OptiMEM (Thermo Fisher) in a total volume of $20 \mathrm{\mu l}$. After a $5 \mathrm{~min}$ incubation period, the transfection mix was added to cells seeded in a 96 -well format. 24 hours posttransfection, the cells were subjected to SARS-CoV-2 infection as described in 'Viral infection and quantification assay in A549-ACE2 cells', or incubated for 72 hours to assess cell viability using the CellTiter-Glo luminescent viability assay according to the manufacturer's protocol (Promega). Luminescence was measured in a Tecan Infinity 2000 plate reader, and percentage viability calculated relative to untreated cells (100\% viability) and cells lysed with $20 \%$ ethanol or $4 \%$ formalin ( $0 \%$ viability), included in each experiment.

\section{Viral infection and quantification assay in A549-ACE2 cells}

Cells seeded in a 96-well format were inoculated with a SARSCoV-2 stock (BetaCoV/France/IDF0372/2020 strain, generated and propagated once in Vero E6 cells and a kind gift from the National Reference Centre for Respiratory Viruses at Institut Pasteur, Paris, originally supplied through the European Virus Archive goes Global platform) at a MOI of 0.1 PFU per cell. Following a one hour incubation period at $37^{\circ} \mathrm{C}$, the virus inoculum was removed, and replaced by DMEM containing $2 \%$ FBS (Gibco, Thermo Fisher). 72 hours post-infection the cell culture supernatant was collected, heat inactivated at $95^{\circ} \mathrm{C}$ for $5 \mathrm{~min}$ and used for RT-qPCR analysis to quantify viral genomes present in the supernatant. Briefly, SARS-CoV-2 specific primers targeting the $\mathrm{N}$ gene region: 5 ' TAATCAGACAAGGAACTGATTA-3' (Forward) and $5^{\prime}$ CGAAGGTGTGACTTCCATG-3' (Reverse) (50) were used with the Luna ${ }^{\circledast}$ Universal One-Step RT-qPCR Kit (New England Biolabs) in an Applied Biosystems QuantStudio 6 thermocycler, with the following cycling conditions: $55^{\circ} \mathrm{C}$ for 10 min, $95^{\circ} \mathrm{C}$ for $1 \mathrm{~min}$, and 40 cycles of $95^{\circ} \mathrm{C}$ for $10 \mathrm{~s}$, followed by $60^{\circ} \mathrm{C}$ for $1 \mathrm{~min}$. The number of viral genomes is expressed as PFU equivalents/ml, and was calculated by performing a standard curve with RNA derived from a viral stock with a known viral titer.

\section{Knockdown validation with qRT-PCR in A549-ACE2 cells}

Gene-specific quantitative PCR primers targeting all genes represented in the OnTargetPlus library were purchased and arrayed in a 96-well format identical to that of the siRNA library (IDT; table S13). A549-ACE2 cells treated with siRNA were lysed using the Luna ${ }^{\circledR}$ Cell Ready Lysis Module (New England Biolabs) following the manufacturer's protocol. The lysate was used directly for gene quantification by RT-qPCR with the Luna ${ }^{\circledast}$ Universal One-Step RT-qPCR Kit (New England Biolabs), using the gene-specific PCR primers and GAPDH as a housekeeping gene. The following cycling conditions were used in an Applied Biosystems QuantStudio 6 thermocycler: $55^{\circ} \mathrm{C}$ for $10 \mathrm{~min}, 95^{\circ} \mathrm{C}$ for $1 \mathrm{~min}$, and 40 cycles of $95^{\circ} \mathrm{C}$ for $10 \mathrm{~s}$, followed by $60^{\circ} \mathrm{C}$ for $1 \mathrm{~min}$. The fold change in gene expression for each gene was derived using the $2^{-\triangle \Delta \mathrm{CT}}$, 2 (Delta Delta CT) method (51), normalized to the constitutively expressed housekeeping gene GAPDH. Relative changes were generated comparing the control siRNA knockdown transfected cells to the cells transfected with each siRNA.

\section{SgRNA Selection for Cas9 knockout screen}

sgRNAs were designed according to Synthego's multi-guide gene knockout (52). Briefly, two or three sgRNAs are bioinformatically designed to work in a cooperative manner to generate small, knockout-causing, fragment deletions in early exons (fig. S18). These fragment deletions are larger than standard indels generated from single guides. The genomic repair patterns from a multi-guide approach are highly predictable based on the guide-spacing and design constraints to limit off-targets, resulting in a higher probability protein knockout phenotype (table S14).

\section{sgRNA Synthesis for Cas9 knockout screen}

RNA oligonucleotides were chemically synthesized on Synthego solid-phase synthesis platform, using CPG solid support containing a universal linker. 5-Benzylthio-1H-tetrazole (BTT, $0.25 \mathrm{M}$ solution in acetonitrile) was used for coupling, (3-((Dimethylamino-methylidene)amino)-3H-1,2,4dithiazole-3-thione (DDTT, $0.1 \mathrm{M}$ solution in pyridine)) was used for thiolation, dichloroacetic acid (DCA, 3\% solution in toluene) was used for detritylation. Modified sgRNA were chemically synthesized to contain 2'-O-methyl analogs and 3' phosphorothioate nucleotide interlinkages in the terminal three nucleotides at both $5^{\prime}$ and $3^{\prime}$ ends of the RNA molecule. After synthesis, oligonucleotides were subject to a series of deprotection steps, followed by purification by solid phase 
extraction (SPE). Purified oligonucleotides were analyzed by ESI-MS.

\section{Arrayed Knockout Generation with Cas9-RNPs}

For Caco-2 transfection, 10 pmol Streptococcus Pyogenes NLS-Sp.Cas9-NLS (SpCas9) nuclease (Aldevron; 9212) was combined with 30 pmol total synthetic sgRNA (10 pmol each sgRNA, Synthego) to form ribonucleoproteins (RNPs) in 20 $\mu$ l total volume with SF Buffer (Lonza V5SC-2002) and allowed to complex at room temperature for $10 \mathrm{~min}$.

All cells were dissociated into single cells using TrypLE Express (Gibco), resuspended in culture media and counted. 100,000 cells per nucleofection reaction were pelleted by centrifugation at 200xg for $5 \mathrm{~min}$. Following centrifugation, cells were resuspended in transfection buffer according to cell type and diluted to $2 \times 10^{4}$ cells $/ \mu l .5 \mu l$ of cell solution was added to preformed RNP solution and gently mixed. Nucleofections were performed on a Lonza HT 384-well nucleofector system (Lonza, \#AAU-1001) using program CM-150 for Caco-2. Immediately following nucleofection, each reaction was transferred to a tissue-culture treated 96 -well plate containing $100 \mu \mathrm{l}$ normal culture media and seeded at a density of 50,000 cells/well. Transfected cells were incubated following standard protocols.

\section{Quantification of arrayed knockout efficiency}

Two days post-nucleofection, genomic DNA was extracted from cells using DNA QuickExtract (Lucigen, \#QE09050). Briefly, cells were lysed by removal of the spent media followed by addition of $40 \mu \mathrm{l}$ of QuickExtract solution to each well. Once the QuickExtract DNA Extraction Solution was added, the cells were scraped off the plate into the buffer. Following transfer to compatible plates, DNA extract was then incubated at $68^{\circ} \mathrm{C}$ for $15 \mathrm{~min}$ followed by $95^{\circ} \mathrm{C}$ for 10 min in a thermocycler before being stored for downstream analysis.

Amplicons for indel analysis were generated by PCR amplification with NEBNext polymerase (NEB, \#M0541) or AmpliTaq Gold 360 polymerase (Thermo Fisher Scientific, \#4398881) according to the manufacturer's protocol. The primers were designed to create amplicons between 400 - 800 $\mathrm{bp}$, with both primers at least $100 \mathrm{bp}$ distance from any of the sgRNA target sites (table S15). PCR products were cleaned-up and analyzed by Sanger sequencing (Genewiz). Sanger data files and sgRNA target sequences were input into Inference of CRISPR Edits (ICE) analysis (ice.synthego.com) to determine editing efficiency and to quantify generated indels (53). Percentage of alleles edited is expressed as an ice-d score. This score is a measure of how discordant the sanger trace is before vs. after the edit. It is a simple and robust estimate of editing efficiency in a pool, especially suited to highly disruptive editing techniques like multi-guide.

\section{Identification of essential genes for siRNA and Cas9 knockout screen}

Here, we used longitudinal imaging in A549 cells to assess cell viability (fig. S18). For benchmarking, relative cell viability was measured by CellTiter-Glo Luminescent Cell Viability Assay (Promega; G7571) as per manufacturer's instructions. Briefly, two passages post-nucleofection A549 siRNA pools cultured in 96-well tissue-culture treated plates (Corning, \#3595) were lysed in the CellTIter-Glo reagent, by removing spent media and adding $100 \mu \mathrm{l}$ of the CellTiter-Glo reagent containing the CellTiter-Glo buffer and CellTiter-Glo Substrate. Cells were placed on an orbital shaker for $2 \mathrm{~min}$ on a SpectraMax iD5 (Molecular Devices) and then incubated in the dark at room temperature for $10 \mathrm{~min}$. Completely lysed cells were pipette mixed and $25 \mu \mathrm{l}$ were transferred to a 384well assay plate (Corning, \#3542). The luminescence was recorded on a SpectraMax iD5 (Molecular Devices) with an integration time of $0.25 \mathrm{~s}$ per well. Luminescence readings were all normalized to the without-sgRNA control condition.

To determine cell viability in Caco-2 knockouts we used longitudinal imaging (fig. S18). All gene knockout pools were maintained for a minimum of six passages to determine the effect of loss of protein function on cell fitness prior to viral infection. Viability was determined through longitudinal imaging and automated image analysis using a Celigo Imaging Cytometer (Celigo). Each gene knockout pool was split in triplicate wells on separate plates. Every day, except the day of seeding, each well was scanned and analyzed using built in 'Confluence' imaging parameters using auto-exposure and autofocus with an offset of $-45 \mu \mathrm{m}$. Analysis was performed with standard settings except for an intensity threshold setting of 8 . Confluency was averaged across 3 wells and plotted over time. Viability genes were determined as pools that were less than $20 \%$ confluent 5 days post seeding following 6 passages. Genes deemed essential were excluded from the knockout screen.

\section{Cells, virus, and infections for Caco-2 Cas9 knockout screen}

Wild-type and CRISPR edited Caco-2 cells were grown at $37^{\circ} \mathrm{C}, 5 \% \mathrm{CO}_{2}$ in DMEM, $10 \%$ FBS. SARS-CoV-2 stocks were grown and titered on Vero E6 cells as described previously (54). Wild-type and CRISPR edited Caco-2 cell lines were infected with SARS-CoV-2 at an MOI of 0.01 in DMEM supplemented with $2 \%$ FBS. 72 hours post-infection, supernatants were harvested and stored at $-80^{\circ} \mathrm{C}$ and the Caco-2 WT/CRISPR KO cells were fixed with $10 \%$ neutral buffered formalin (NBF) for 1 hour at room temperature to enable further analysis.

Focus forming assay for Caco-2 Cas9 knockout screen Vero E6 cells were plated into 96 well plates at confluence 
(50,000 cells/well) in DMEM supplemented with $10 \%$ heatinactivated FBS (Gibco). Prior to infection, supernatants from infected Caco-2 WT/CRISPR KO cells were thawed and serially diluted from $10^{-1}$ to $10^{-8}$. Growth media was removed from the Vero E6 cells and $40 \mu \mathrm{l}$ of each virus dilution was plated. After 1 hour adsorption at $37^{\circ} \mathrm{C}, 5 \% \mathrm{CO}_{2}, 40 \mu \mathrm{l}$ of $2.4 \%$ microcrystalline cellulose (MCC) overlay supplemented with DMEM powdered media (Gibco) to a concentration of $1 \mathrm{x}$ was added to each well of the 96 well plate to achieve a final MCC overlay concentration of $1.2 \%$. Plates were then incubated at $37^{\circ} \mathrm{C}, 5 \% \mathrm{CO}_{2}$ for 24 hours. The MCC overlay was gently removed and cells were fixed with $10 \% \mathrm{NBF}$ for 1 hour at roomtemperature. After removal of NBF, monolayers were washed with ultrapure water and ice-cold $100 \%$ methanol/0.3\% $\mathrm{H}_{2} \mathrm{O}_{2}$ was added for $30 \mathrm{~min}$ to permeabilize the cells and quench endogenous peroxidase activity. Monolayers were then blocked for 1 hour in PBS with 5\% non-fat dry milk (NFDM). After blocking, monolayers were incubated with SARS-CoV N primary antibody (Novus Biologicals; NB100-56576 - 1:2000) for 1 hour at room temperature in PBS, 5\% NFDM. Monolayers were washed with PBS and incubated with an HRPConjugated secondary antibody for 1 hour at room temperature in PBS with 5\% NFDM. Secondary was removed, monolayers were washed with PBS, and then developed using TrueBlue substrate (KPL) for $30 \mathrm{~min}$. Plates were imaged on a Bio-Rad Chemidoc utilizing a phosphorscreen and foci were counted by eye to calculate focus forming units per $\mathrm{ml}$ (FFU/ml) for each knockout. The original formalin-fixed Caco-2 WT/CRISPR KO cells were stained with Dapi (Thermo Scientific) and imaged on a Cytation 5 plate reader to determine cell viability. Wells containing no cells were excluded from further analyses.

\section{Quantitative analysis and scoring of knockdown and knockout library screens}

Virus readout by qPCR (A549-ACE2, expressed as PFU/ml) and focus forming assay readouts (Caco-2, FFU/ml) were processed using the RNAither package (https://www.bioconductor.org/packages/release/bioc/html/ RNAither.html) in the statistical computing environment $\mathrm{R}$. The two datasets were normalized separately, using the following method. The readouts were first log transformed (natural logarithm), and robust Z-scores (using median and MAD "median absolute deviation" instead of mean and standard deviation) were then calculated for each 96-well plate separately. Z-scores of multiple replicates of the same perturbation were averaged into a final Z-score for presentation in Fig. 5. No filtering was done based on differences in replicate Zscores, but all replicate scores are individually listed in tables $\mathrm{S} 6$ and S7. We suggest consulting the replicate Z-scores for all genes/perturbations of interest. The A549-ACE2 siRNA screen includes 3 replicates (or more) of each perturbation, and the Caco-2 CRISPR screen includes 2 replicates (or more) of each perturbation. The results from the A549-ACE2 screen cover all 332 screened genes (331 SARS-CoV-2 interactors plus ACE2). The results from the Caco-2 screen cover 286 of the screened genes plus ACE2. The remaining Caco-2 genes were either deemed essential, failed editing, or failed in the focus forming assay.

\section{Antiviral drug and cytotoxicity assays (A549-ACE2 cells)}

2,500 A549-ACE2 cells were seeded into 96- or 384-well plates in DMEM (10\% FBS) and incubated for 24 hours at $37^{\circ} \mathrm{C}, 5 \%$ $\mathrm{CO}_{2}$. Two hours prior to infection, the media was replaced with $120 \mu \mathrm{l}$ (96 well format) or $50 \mu \mathrm{l}$ (384 well format) of DMEM (2\% FBS) containing the compound of interest at the indicated concentration. At the time of infection, the media was replaced with virus inoculum (MOI 0.1 PFU/cell) and incubated for 1 hour at $37^{\circ} \mathrm{C}, 5 \% \mathrm{CO}_{2}$. Following the adsorption period, the inoculum was removed, replaced with $120 \mu \mathrm{l}(96$ well format) or $50 \mu \mathrm{l}$ (384 well format) of drug-containing media, and cells incubated for an additional 72 hours at $37^{\circ} \mathrm{C}$, $5 \% \mathrm{CO}_{2}$. At this point, the cell culture supernatant was harvested, and viral load assessed by RT-qPCR (as described in 'Viral infection and quantification assay in A549-ACE2 cells'). Viability was assayed using the CellTiter-Glo assay following the manufacturer's protocol (Promega). Luminescence was measured in a Tecan Infinity 2000 plate reader, and percentage viability calculated relative to untreated cells ( $100 \%$ viability) and cells lysed with $20 \%$ ethanol or $4 \%$ formalin $(0 \%$ viability), included in each experiment.

\section{Antiviral drug and cytotoxicity assays (Vero E6 cells)}

Viral growth and cytotoxicity assays in the presence of inhibitors were performed as previously described (5). 2,000 Vero E6 cells were seeded into 96-well plates in DMEM (10\% FBS) and incubated for 24 hours at $37^{\circ} \mathrm{C}, 5 \% \mathrm{CO}_{2}$. Two hours before infection, the medium was replaced with $100 \mu \mathrm{l}$ of DMEM ( $2 \% \mathrm{FBS}$ ) containing the compound of interest at concentrations $50 \%$ greater than those indicated, including a DMSO control. SARS-CoV-2 virus (100 PFU; MOI 0.025) was added in $50 \mu \mathrm{l}$ of DMEM (2\% FBS), bringing the final compound concentration to those indicated. Plates were then incubated for 48 hours at $37^{\circ} \mathrm{C}$. After infection, supernatants were removed and cells were fixed with $4 \%$ formaldehyde for 24 hours prior to being removed from the BSL3 facility. The cells were then immunostained for the viral NP protein (rabbit anti-sera produced in the Garcia-Sastre lab; 1:10,000) with a DAPI counterstain. Infected cells $(488 \mathrm{~nm})$ and total cells (DAPI) were quantified using a Celigo (Nexcelcom) imaging cytometer. Infectivity is measured by the accumulation of viral NP protein in the nucleus of the cells (fluorescence accumulation). Percent infection was quantified as (Infected cells 
/ Total cells) - Background) * 100 and the DMSO control was then set to $100 \%$ infection for analysis. The $\mathrm{IC}_{50}$ and $\mathrm{IC}_{90}$ for each experiment was determined using the Prism (GraphPad Software) software. Cytotoxicity measurements were performed using the MTT assay (Roche), according to the manufacturer's instructions. Cytotoxicity was performed in uninfected Vero E6 cells with same compound dilutions and concurrent with viral replication assay. All assays were performed in biologically independent triplicates. Sourcing information for all drugs tested may be found in table S10.

\section{Co-immunoprecipitation assays for Orf9b and Tom70}

HEK293T and A549 cells were transfected with the indicated mammalian expression plasmids using Lipofectamine 2000 (Invitrogen) and TransIT-X2 (Mirus Bio) respectively. 24 hours post-transfection, cells were harvested and lysed in NP40 lysis buffer $(0.5 \%$ Nonidet P 40 Substitute (NP-40; Fluka Analytical), $50 \mathrm{mM}$ Tris- $\mathrm{HCl}, \mathrm{pH} 7.4$ at $4^{\circ} \mathrm{C}, 150 \mathrm{mM} \mathrm{NaCl}, 1$ mM EDTA) supplemented with cOmplete mini EDTA-free protease and PhosSTOP phosphatase inhibitor cocktails (Roche). Clarified cell lysates were incubated with Streptactin Sepharose beads (IBA) for 2 hours at $4^{\circ} \mathrm{C}$, followed by five washes with NP-40 lysis buffer. Protein complexes were eluted in the SDS loading buffer and were analyzed by Western blotting with the indicated antibodies.

\section{Quantification of Tom7o down-regulation in HeLaM cells overexpressing $\mathrm{Orf9b}$}

HeLaM cells were transiently transfected with plasmids encoding GFP-Strep, SARS-CoV-1 Orf9b-Strep or SARS-CoV-2 Orf9b-Strep. The next day, the cells were fixed using $4 \%$ paraformaldehyde and immunostained with antibodies against Strep tag, and Tom20 or Tom70. Representative images for each construct were captured by acquiring a single optical section using a Nikon A1 confocal fitted with a CFI Plan Apochromat VC 60x oil objective (NA 1.4). For image quantification multiple fields of view were captured for each construct using a CFI Super Plan Fluor ELWD 40x objective (NA 0.6). The mean fluorescent intensity for Tom 20 and Tom70 was measured by manually drawing a region of interest around each cell using ImageJ. Between 30 and 60 cells were quantified for each construct.

\section{Quantification of Tom7o down-regulation in infected Caco-2 cells}

Caco-2 cells were seeded on glass coverslips in triplicate and infected with SARS-CoV-2 at an MOI of 0.1 as described above. At 24 hours post-infection, cells were fixed with $4 \%$ paraformaldehyde and immunostained with antibodies against Tom70, Tom20 and Orf9b. For signal quantification images of non-infected and neighboring infected cells were acquired using a LSM800 confocal laser-scanning microscope
(Zeiss) equipped with a 63X, 1.4 NA oil objective and the Zen blue software (Zeiss). The mean fluorescence intensity of each cell was measured by ImageJ software. 43 cells were quantified for each condition, infected or non-infected, from three independent experiments.

\section{Co-expression and Purification of Orf9b-Tom7o (109- end) complexes}

SARS-CoV-2 Orf9b and Tom70 (residues 109-end) were coexpressed using a pET29-b(+) vector backbone where Orf9b was tag-less and Tom70 had an N-terminal 10XHis-tag and SUMO-tag. LOBSTR $E$. coli cells transformed with the above construct were grown at $37^{\circ} \mathrm{C}$ till O.D. $(600 \mathrm{~nm})=0.8$ and the expression was induced at $37^{\circ} \mathrm{C}$ with $1 \mathrm{mM}$ IPTG for 4 hours. Frozen cell pellets were resuspended in $25 \mathrm{ml}$ lysis buffer (200 mM NaCl, $50 \mathrm{mM}$ Tris-HCl pH 8.0, 10\% v/v glycerol, 2 $\mathrm{mM} \mathrm{MgCl}_{2}$ ) per liter cell culture, supplemented with cOmplete protease inhibitor tablets (Roche), $1 \mathrm{mM}$ PMSF (Sigma), $100 \mu \mathrm{g} / \mathrm{ml}$ lysozyme (Sigma), $5 \mu \mathrm{g} / \mathrm{ml}$ DNaseI (Sigma), and then homogenized with an immersion blender (Cuisinart). Cells were lysed by 3x passage through an Emulsiflex C 3 cell disruptor (Avestin) at $\sim 15,000 \mathrm{psi}$, and the lysate clarified by ultracentrifugation at $100,000 \times \mathrm{xg}$ for $30 \mathrm{~min}$ at $4{ }^{\circ} \mathrm{C}$. The supernatant was collected, supplemented with $20 \mathrm{mM}$ imidazole, loaded into a gravity flow column containing Ni-NTA superflow resin (Qiagen), and rocked with the resin at $4{ }^{\circ} \mathrm{C}$ for 1 hour. After allowing the column to drain, resin was rinsed twice with 5 column volumes (cv) of wash buffer $(150 \mathrm{mM}$ $\mathrm{KCl}, 30 \mathrm{mM}$ Tris- $\mathrm{HCl} \mathrm{pH}$ 8.0, 10\% v/v glycerol, $20 \mathrm{mM}$ imidazole, $0.5 \mathrm{mM}$ tris(hydroxypropyl)phosphine (THP, VWR)) supplemented with $2 \mathrm{mM}$ ATP (Sigma) and $4 \mathrm{mM} \mathrm{MgCl}_{2}$, then washed with $5 \mathrm{cv}$ wash buffer with $40 \mathrm{mM}$ imidazole. Resin was then rinsed with $5 \mathrm{cv}$ Buffer A $(50 \mathrm{mM} \mathrm{KCl}, 30 \mathrm{mM}$ Tris$\mathrm{HCl} \mathrm{pH} \mathrm{8.0,5 \%} \mathrm{glycerol,} 0.5 \mathrm{mM} \mathrm{THP}$ ) and protein was eluted with $2 \times 2.5$ cv Buffer A $+300 \mathrm{mM}$ imidazole. Elution fractions were combined, supplemented with Ulp1 protease, and rocked at $4{ }^{\circ} \mathrm{C}$ for 2 hours. Ulp1-digested Ni-NTA eluate was diluted 1:1 with additional Buffer A, loaded into a $50 \mathrm{ml} \mathrm{Su}$ perloop, and applied to a MonoQ 10/100 column on an Äkta pure system (GE Healthcare) using 100\% Buffer A, 0\% Buffer B (1000 mM KCl, $30 \mathrm{mM}$ Tris- $\mathrm{HCl} \mathrm{pH}$ 8.0, 5\% glycerol, 0.5 $\mathrm{mM}$ THP). The MonoQ column was washed with $0 \%-40 \%$ Buffer B gradient over $15 \mathrm{cv}$, peak fractions were analyzed by SDS-PAGE and the identity of tagless Tom70(109-end) and Orf9b proteins confirmed by intact protein mass spectrometry (Xevo G2-XS Mass Spectrometer, Waters). Peak fractions eluting at $\sim 15 \%$ B contained relatively pure Tom70(109-end) and Orf9b, and these were concentrated using 10kDa Amicon centrifugal filter (Millipore) and further purified by size exclusion chromatography using a Superdex 200 increase 10/300 GL column (GE healthcare) in buffer containing 150 $\mathrm{mM} \mathrm{KCl}, 20 \mathrm{mM}$ HEPES-NaOH pH 7.5, $0.5 \mathrm{mM}$ THP. The sole 
size-exclusion peak contained both Tom70(109-end) and Orf9b, and the center fraction was used directly for cryo-EM grid preparation.

\section{Expression and Purification of SARS-CoV-2 Orf9b}

Orf9b with N-terminal 10XHis-tag and SUMO-tag was expressed using a pET-29b(+) vector backbone. LOBSTR E. coli cells transformed with the above construct were grown at $37^{\circ} \mathrm{C}$ until reaching O.D. $(600 \mathrm{~nm})=0.8$ and the expression was induced at $37^{\circ} \mathrm{C}$ with $1 \mathrm{mM}$ IPTG for 6 hours. Frozen cell pellets were lysed, homogenized, clarified, and subject to $\mathrm{Ni}$ affinity purification as described above for Orf9b-Tom70 complexes, with several small changes. Lysis buffers and NiNTA wash buffers contained $500 \mathrm{mM} \mathrm{NaCl}$, and an additional wash step using $10 \mathrm{cv}$ wash buffer $+0.2 \%$ TWEEN20 + 500 $\mathrm{mM} \mathrm{NaCl}$ was carried out prior to the ATP wash. Orf9b was eluted from Ni-NTA resin in Buffer A ( $50 \mathrm{mM} \mathrm{NaCl}, 25 \mathrm{mM}$ Tris pH 8.5, $5 \%$ glycerol, $0.5 \mathrm{mM}$ THP) supplemented with $300 \mathrm{mM}$ imidazole. This eluate was diluted 1:1 with additional Buffer A, loaded into a $50 \mathrm{ml}$ Superloop, and applied to a MonoQ 10/100 column on an Äkta pure system (GE Healthcare) using $100 \%$ Buffer A, 0\% Buffer B (1000 mM $\mathrm{NaCl}, 25 \mathrm{mM}$ Tris- $\mathrm{HCl} \mathrm{pH} 8.5,5 \%$ glycerol, $0.5 \mathrm{mM}$ THP). The MonoQ column was washed with $0 \%-40 \%$ Buffer B gradient over $15 \mathrm{cv}$, and relatively pure Orf9b eluted at 20-25\% Buffer $\mathrm{B}$, whereas Orf9b and contaminating proteins eluted at 30$35 \%$ buffer B. Fractions from these two peaks were combined and incubated with Ulp1 and HRV3C proteases at $4{ }^{\circ} \mathrm{C}$ for 2 hours, supplemented with $10 \mathrm{mM}$ imidazole, then thrice flowed back through $1 \mathrm{ml}$ of Ni-NTA resin equilibrated with size-exclusion buffer (as above) $+10 \mathrm{mM}$ imidazole. The reverse-Ni purified sample was concentrated using $10 \mathrm{kDa}$ Amicon centrifugal filter and then further purified by size exclusion chromatography using a Superdex 200 increase 10/300 GL column.

\section{Expression and Purification of Tom70(109-end)}

Tom70 (109-end) with N-terminal 10XHis-tag and SUMO-tag and $\mathrm{C}$ terminus Spy-tag, HRV-3C protease cleavage site, and eGFP-tag was expressed using a pET-21(+) vector backbone. LOBSTR $E$. coli cells transformed with the above construct were grown at $37^{\circ} \mathrm{C}$ till O.D. $(600 \mathrm{~nm})=0.8$ and the expression was induced at $16^{\circ} \mathrm{C}$ with $0.5 \mathrm{mM}$ IPTG overnight. The soluble domain of Tom70 (Tom70 (109-end)) was purified as described in (55) with some modifications. Frozen cell pellets of LOBSTR $E$. coli transformed with the above construct were resuspended in $50 \mathrm{ml}$ lysis buffer $(500 \mathrm{mM} \mathrm{NaCl}, 20 \mathrm{mM}$ $\mathrm{KH}_{2} \mathrm{PO}_{4} \mathrm{pH}$ 7.5) per liter cell culture, supplemented with 1 $\mathrm{mM}$ PMSF (Sigma) and $100 \mu \mathrm{g} / \mathrm{ml}$, and homogenized. Cells were lysed by 3x passage through an Emulsiflex C3 cell disruptor (Avestin) at $\sim 15,000 \mathrm{psi}$, and the lysate clarified by ultracentrifugation at $100,000 \times \mathrm{xg}$ for $30 \mathrm{~min}$ at $4^{\circ} \mathrm{C}$. The supernatant was collected, supplemented with $20 \mathrm{mM}$ imidazole, loaded into a gravity flow column containing Ni-NTA superflow resin (Qiagen), and rocked with the resin at $4^{\circ} \mathrm{C}$ for 1 hour. After allowing the column to drain, resin was rinsed with twice with 5 column volumes (cv) of wash buffer (500 $\mathrm{mM} \mathrm{KCl}, 20 \mathrm{mM} \mathrm{KH}_{2} \mathrm{PO}_{4} \mathrm{pH} 8.0,20 \mathrm{mM}$ imidazole, $0.5 \mathrm{mM}$ THP) supplemented with $2 \mathrm{mM}$ ATP - $4 \mathrm{mM} \mathrm{MgCl} 2$, then washed with $5 \mathrm{cr}$ wash buffer with $40 \mathrm{mM}$ imidazole. Bound Tom70(109-end) was then cleaved from the resin by 2 hour incubation with Ulp1 protease in $4 \mathrm{cv}$ elution buffer $(150 \mathrm{mM}$ $\mathrm{KCl}, 20 \mathrm{mM} \mathrm{KH} \mathrm{PO}_{4} \mathrm{pH}$ 8.0, $5 \mathrm{mM}$ imidazole, $0.5 \mathrm{mM} \mathrm{THP}$ ). After cleavage with Ulp1, the flow through was collected along with a $2 \mathrm{cv}$ rinse of the resin with additional elution buffer. These fractions were combined and HRV3C protease was added to remove the C-terminal EGFP tag (1:20 HRV3C to Tom70). After 2 hour HRV3C digestion at $4{ }^{\circ} \mathrm{C}$, the doubledigested Tom70(109-end) was concentrated using a $30 \mathrm{kDa}$ Amicon centrifugal filter (Millipore) and further purified by size exclusion chromatography using a Superdex 200 increase 10/300 GL column (GE healthcare) in buffer containing $150 \mathrm{mM} \mathrm{KCl}, 20 \mathrm{mM}$ HEPES-NaOH pH 7.5, $0.5 \mathrm{mM}$ THP.

\section{Prediction of SARS-CoV-2 Orf9b internal mitochon- drial targeting sequence}

Orf9b was analyzed for the presence of an internal mitochondrial targeting sequence (i-MTS) as described in (56) using the TargetP-2.0 server (57). Sequences corresponding to Orf9b N-terminal truncations of 0 to 62 residues were submitted to the TargetP-2.0 server, and the probability of the peptides containing an MTS plotted against the numbers of residues truncated. A similar analysis using the MitoFates server (58) predicted that Orf9b residues 54-63 were the most likely to comprise a presequence MTS based on propensity to form a positively charged amphipathic helix. Notably this analysis was consistent with the secondary structure prediction from JPRED (59).

\section{CryoEM sample preparation and data collection}

$3 \mu \mathrm{L}$ of Orf9b-Tom70 complex $(12.5 \mu \mathrm{M})$ was added to a 400 mesh 1.2/1.3R Au Quantifoil grid previously glow discharged at $15 \mathrm{~mA}$ for $30 \mathrm{~s}$. Blotting was performed with a blot force of 0 for $5 \mathrm{~s}$ at $4^{\circ} \mathrm{C}$ and $100 \%$ humidity in a FEI Vitrobot Mark IV (ThermoFisher) prior to plunge freezing into liquid ethane. 1534 118-frame super-resolution movies were collected with a $3 \times 3$ image shift collection strategy at a nominal magnification of 105,000x (physical pixel size: $0.834 \AA \AA$ pix) on a Titan Krios (ThermoFisher) equipped with a K3 camera and a Bioquantum energy filter (Gatan) set to a slit width of $20 \mathrm{eV}$. Collection dose rate was $8 \mathrm{e}-/ \mathrm{pixel} / \mathrm{second}$ for a total dose of $66 \mathrm{e}-$ /Å2. Defocus range was -0.7um to -2.4um. Each collection was performed with semi-automated scripts in SerialEM (60). 


\section{CryoEM Image Processing and Model Building}

1534 movies were motion corrected using Motioncor2 (61) and dose-weighted summed micrographs were imported in cryosparc (v2.15.0). 1427 micrographs were curated based on CTF fit (better than $5 \AA$ Å) from a patch CTF job. Templatebased particle picking resulted in 2,805,121 particles and 1,616,691 particles were selected after 2D-classification. Five rounds of 3D-classification using multi-class ab-initio reconstruction and heterogeneous refinement yielded 178,373 particles. Homogenous refinement of these final particles led to a 3.1 Å electron density map which was used for model building. The reconstruction was filtered by the masked FSC and sharpened with a b-factor of - 145 .

To build the model of Tom70(109-end), the crystal structure of Saccharomyces cerevisiae Tom71 (PDB ID: 3fp3; sequence identity $25.7 \%$ ) was first fit into the cryoEM density as a rigid body in UCSF ChimeraX and then relaxed into the final density using Rosetta FastRelax mover in torsion space. This model, along with a BLAST alignment of the two sequences (62), was used as a starting point for manual building using COOT (63). After initial building by hand the regions with poor density fit/geometry were iteratively rebuilt using Rosetta (64). Orf9b was built de novo into the final density using COOT, informed and facilitated by the predictions of the TargetP-2.0, MitoFates, and JPRED servers. The Orf9b-Tom70 complex model was submitted to the Namdinator web server (65) and further refined in ISOLDE 1.0 (66) using the plugin for UCSF ChimeraX (67). Final model B-factors were estimated using Rosetta. The model was validated using phenix.validation_cryoem (68). The final model contains residues 109-272, 298-600 of human Tom70, and 39-76 of SARS-CoV-2 Orf9b. Molecular interface between Orf9b and Tom70 was analyzed using the PISA web server (69). Figures were prepared using UCSF ChimeraX.

\section{Computational human genetics analysis}

To look for genetic variants associated with our list of proteins that had a significant impact on SARS-CoV-2 replication, we used the largest proteomic GWAS study to date (70). We identified IL17RA as one of the proteins assayed in Sun $e t$ al.'s proteomic GWAS and observed that it had multiple cisacting protein quantitative trait loci (pQTLs) at a corrected p-value $1 \times 10^{-5}$, where cis-acting is defined as within $1 \mathrm{MB}$ of the transcription start site of IL17RA.

We used the GSMR method (71) to perform MR using near-independent (linkage disequilibrium or $\mathrm{LD} \mathrm{r}^{2}=0.05$ ) cis-pQTLs for IL17RA. The advantage of GSMR method over conventional MR methods is two-fold; first, GSMR performs MR adjusting for any residual correlation between selected genetic variants by default. Second, GSMR has a built-in method called HEIDI (heterogeneity in dependent instruments)-outlier that performs heterogeneity tests in the near- independent genetic instruments and remove potentially pleiotropic instruments (i.e., where there is evidence of heterogeneity at $\mathrm{p}<0.01)$. Details of the GSMR and HEIDI method have been published previously (71).

Summary statistics generated by COVID-19 Human Genetics Initiative (COVID-HGI) (round 3; https://www.covid19hg.org/results/) for COVID-19 vs. population, hospitalized COVID-19 vs. population and hospitalized COVID-19 vs. non-hospitalized COVID-19 were used for IL17RA MR analysis. We used the 1000 genomes phase $3 \mathrm{Eu}-$ ropean population genotype data to derive the LD correlation matrix for this analysis. The phenotype definitions as provided by COVID-HGI are as follows. COVID-19 vs. population: Case, individuals with laboratory confirmation of SARS-CoV2 infection, EHR/ICD coding/Physician-confirmed COVID-19, or self-reported COVID-19 positive; control, everybody that is not a case. Hospitalized COVID-19 vs. population: case, hospitalized, laboratory confirmed SARS-CoV-2 infection or hospitalization due to COVID-19-related symptoms; control, everybody that is not a case, e.g., population. Hospitalized COVID-19 vs. non-hospitalized COVID-19: case, hospitalized, laboratory confirmed SARS-CoV-2 infection or hospitalization due to COVID-19-related symptoms; control, laboratory confirmed SARS-CoV-2 infection and not hospitalized 21 days after the test.

\section{Infections and treatments for IL17A treatment studies}

The WA-1 strain (BEI resources) of SARS-CoV-2 was used for all experiments. All live virus experiments were performed in a BSL3 lab. SARS-CoV-2 stocks were passaged in Vero E6 cells (ATCC) and titer was determined via plaque assay on Vero E6 cells as previously described (72). Briefly, virus was diluted 1:10 $-1: 10^{6}$ and incubated for 1 hour on Vero E6 cells before an overlay of Avicel and complete DMEM (Sigma Aldrich, SLM-241) was added. After incubation at $37^{\circ} \mathrm{C}$ for 72 hours, the overlay was removed and cells were fixed with $10 \%$ formalin, stained with crystal violet, and counted for plaque formation. SARS-CoV-2 infections of A549-ACE2 cells were done at a MOI of 0.05 for 24 hours. Inhibitors and cytokines were added concurrently with virus. All infections were done in technical triplicate. Cells were treated with the following compounds: Remdesivir (SELLECK CHEMICALS LLC, S8932) and IL-17A (Millipore-Sigma, SRP0675).

\section{RNA extraction, $R T$, and quantitative RT-PCR for IL17A treatment studies}

Total RNA from samples was extracted using the Direct-zol RNA kit (Zymogen, R2060) and quantified using the NanoDrop 2000c (ThermoFisher). cDNA was generated using 500 ng of RNA from infected A549-ACE2 cells with Superscript III reverse transcription (ThermoFisher, 18080-044) and oligo(dT) $)_{12-18}$ (ThermoFisher, 18418-012) and random 
hexamer primers (ThermoFisher, S0142). Quantitative RTPCR reactions were performed on a CFX384 (BioRad) and delta cycle threshold $(\triangle \mathrm{Ct})$ was determined relative to RPL13A levels. Viral detection levels and target host genes in treated samples were normalized to water-treated controls. The SYBR green qPCR reactions contained $5 \mu$ l of 2x Maxima SYBR green/Rox qPCR Master Mix (ThermoFisher; K0221), 2 $\mu \mathrm{l}$ of diluted cDNA, and $1 \mathrm{nmol}$ of both forward and reverse primers, in a total volume of $10 \mu \mathrm{l}$. The reactions were run as follows: $50^{\circ} \mathrm{C}$ for $2 \mathrm{~min}$ and $95^{\circ} \mathrm{C}$ for $10 \mathrm{~min}$, followed by 40 cycles of $95^{\circ} \mathrm{C}$ for $5 \mathrm{~s}$ and $62^{\circ} \mathrm{C}$ for $30 \mathrm{~s}$. Primer efficiencies were around $100 \%$. Dissociation curve analysis after the end of the PCR confirmed the presence of a single and specific product. qRT-PCR primers were used against the SARS-CoV2 E gene (PF_042_nCoV_E_F: ACAGGTACGTTAATAGTTAATAGCGT; PF_042_nCoV_E_R: ATATTGCAGCAGTACGCACACA), the CXCL8 gene (CXCL8 For: ACTGAGAGTGATTGAGAGTGGAC; CXCL8 Rev: AACCCTCTGCACCCAGTTTTC), and the RPL13A gene (RPL13A For: CCTGGAGGAGAAGAGGAAAGAGA; RPL13A Rev: TTGAGGACCTCTGTGTATTTGTCAA).

\section{Transfections for IL17A treatment studies}

HEK293T cells were seeded $5 \times 10^{5}$ cells/well (in 6 well plate) or $3 \times 10^{6}$ cell $/ 10 \mathrm{~cm}^{2}$ plates. Next day, $2 \mu \mathrm{g}$ or $10 \mu \mathrm{g}$ of plasmids was transfected using X-tremeGENE 9 DNA Transfection Reagent (Roche) in 6 well plate or $10 \mathrm{~cm}^{2}$ plates respectively. For IL-17A (Millipore-Sigma, SRP0675) incubation in cells, $0.5 \mu \mathrm{g}$ of IL-17A was treated either pre- or post-transfection and incubated at $37^{\circ} \mathrm{C}$. After 48 hours, cells were collected by trypsinization. For IL-17A incubation with cell lysates, transfected cell lysates were incubated with presence of 0.5 and $5 \mu \mathrm{g} / \mathrm{ml}$ IL-17A at $4^{\circ} \mathrm{C}$ on rotation overnight. Plasmids pLVXEF1alpha-SARS-CoV-2-orf8-2xStrep-IRES-Puro (Orf8) and pLVX-EF1alpha-eGFP-2xStrep-IRES-Puro (EGFP-Strep) were a gift from Nevan Krogan. (Addgene plasmid \#141390, 141395) (5). pLVX-EF1alpha- IRES-Puro (Vector) was obtained from Takara/Clontech.

\section{SARS-CoV-2 Orf8 and IL17RA Co-immunoprecipitation} Transfected and treated HEK293T cells were pelleted and washed in cold D-PBS and later resuspended in Flag-IP Buffer (50 mM Tris HCl, pH 7.4, with $150 \mathrm{mM} \mathrm{NaCl}, 1$ mM EDTA, and $1 \%$ NP-40) with 1x HALT (ThermoFisher Scientific, 78429), incubated with buffer for $15 \mathrm{~min}$ on ice then centrifuged at 13,000 rpm for $5 \mathrm{~min}$. The supernatant was collected and $1 \mathrm{mg}$ of protein was used for Immunoprecipitation (IP) with $100 \mu \mathrm{l}$ Streptactin Sepharose (IBA, 2-1201-010) on a rotor overnight at $4{ }^{\circ} \mathrm{C}$. Immunoprecipitates were washed 5 times with Flag-IP buffer and eluted with 1x Buffer E (100 mM Tris$\mathrm{Cl}, 150 \mathrm{mM} \mathrm{NaCl}, 1 \mathrm{mM}$ EDTA, $2.5 \mathrm{mM}$ Desthiobiotin). Eluate was diluted with 1x-NuPAGE (ThermoFisher Scientific,
\#NP0008) LDS Sample Buffer with 2.5\% $\beta$-Mercaptoethanol and blotted for targeted antibodies. Antibodies used were Strep Tag II (Qiagen, \#34850), B-Actin (Sigma, \#A5316), and IL17RA (Cell Signaling, \#12661S).

\section{Computational docking of mPGES-2 and Nsp7}

A model for human mPGES-2 dimer was constructed by homology using MODELER (73) from the crystal structure of Macaca fascularis mPGES-2 (PDB 1Z9H (74), 98\% sequence identity) bound to indomethacin. Indomethacin was removed from the structure utilized for docking. The structure of SARS-CoV-2 Nsp7 was extracted from PDB 7BV2 (75). Docking models were produced using ClusPro (76), ZDock (77), HDock (78), Gramm-X (79), SwarmDock (80) and PatchDock (81) with SOAP-PP score (82). For each protocol, up to 100 top scoring models were extracted (fewer for those that do not report > 100 models); for PatchDock, models with SOAP-PP Z-scores greater than 3.0 were used (fig. S23A). The 420 models were clustered at $4.0 \AA$ RMSD, resulting in 127 clusters. The two largest clusters, comprising 192 models, are related by the dimer symmetry. All other clusters contain fewer than 15 models.

\section{Assessment of positive selection signatures in SIGMAR1}

SIGMAR1 protein alignments were generated from whole genome sequences of 359 mammals curated by the Zoonomia consortium. Protein alignments were generated with TOGA (https://github.com/hillerlab/TOGA), and missing sequence gaps were refined with CACTUS $(83,84)$. Branches undergoing positive selection were detected with the branch-site test aBSREL (85) implemented in the HyPhy package $(85,86)$. PhyloP was used to detect codons undergoing accelerated evolution along branches detected as undergoing positive selection by aBSREL relative to the neutral evolution rate in mammals, determined using phyloFit on third nucleotide positions of codons which are assumed to evolve neutrally. Pvalues from phyloP were corrected for multiple tests using the Benjamini-Hochberg method (87). PhyloFit and phyloP are both part of the PHAST package v1.4 $(88,89)$.

\section{Comparative SARS-CoV-1 inhibition by amiodarone}

SARS-CoV-1 (Urbani) drug screens were performed with Vero E6 cells (ATCC\# 1568, Manassas, VA) cultured in DMEM (Quality Biological), supplemented with $10 \%$ (v/v) heat inactivated fetal bovine serum (Sigma), 1\% (v/v) penicillin/streptomycin (Gemini Bio-products), and 1\% (v/v) L-glutamine (2 $\mathrm{mM}$ final concentration, Gibco). Cells were plated in opaque 96 well plates one day prior to infection. Drugs were diluted from stock to $50 \mu \mathrm{M}$ and an 8-point 1:2 dilution series prepared in duplicate in Vero Media. Every compound dilution and control was normalized to contain the same 
concentration of drug vehicle (e.g., DMSO). Cells were pretreated with drug for 2 hours (h) at $37^{\circ} \mathrm{C}(5 \% \mathrm{CO} 2)$ prior to infection with SARS-CoV-1 at MOI 0.01. In addition to plates that were infected, parallel plates were left uninfected to monitor cytotoxicity of drug alone. All plates were incubated at $37^{\circ} \mathrm{C}$ (5\% CO2) for 3 days before performing CellTiter-Glo (CTG) assays as per the manufacturer's instruction (Promega, Madison, WI). Luminescence was read on a BioTek Synergy HTX plate reader (BioTek Instruments Inc., Winooski, VT) using the Gen5 software (v7.07, Biotek Instruments Inc., Winooski, VT).

\section{Real-world data source and analysis}

This study used de-identified patient-level records from HealthVerity's Marketplace dataset, a nationally representative dataset covering $>300$ million unique patients with medical and pharmacy records from over 60 healthcare data sources in the US. The current study used data from 738,933 patients with documented COVID-19 infection between March 1, 2020 to August 17, 2020, defined as a positive or presumptive positive viral lab test result or an International Classification of Diseases, $10^{\text {th }}$ Revision, Clinical Modification (ICD-10-CM) diagnosis code of U07.1 (COVID-19).

For this population, we analyzed medical claims, pharmacy claims, laboratory data, and hospital chargemaster data containing diagnoses, procedures, medications and COVID19 laboratory results from both inpatient and outpatient settings. Claims data included open (unadjudicated) claims sourced in near-real time from practice management and billing systems, claims clearinghouses and laboratory chains, as well as closed (adjudicated) claims encompassing all major US payer types (commercial, Medicare, Medicaid). For inpatient treatment evaluations, we used linked hospital chargemaster data containing records of all billable procedures, medical services and treatments administered in hospital settings. Linkage of patient-level records across these data types provides a longitudinal view of baseline health status, medication use, and COVID-19 progression for each patient under study. Data for this study covered the period of December 1, 2018 through August $17^{\text {th }}, 2020$. All analyses were conducted with the Aetion Evidence Platform version r4.6.

This study was approved by the New England IRB (\#19757-1). Medical records constitute protected health information and can be made available to qualified individuals upon reasonable request.

\section{Observation of hospitalization outcomes in outpatient new users of indomethacin (treatment arm) vs. celecoxib (active comparator) using real-world data}

We used an incident (new) user, active comparator design $(90,91)$ to assess the risk of hospitalization among newly diagnosed COVID-19 patients who were subsequently treated with indomethacin or the comparator agent, celecoxib. Patients were required to have COVID-19 infection recorded in an outpatient setting during the study period of March 1, 2020 to August 17, 2020 and occurring in the 21 days prior to (and including) the date of indomethacin or celecoxib treatment initiation. Prevalent users of prescription-only NSAIDs (any prescription fill for indomethacin, celecoxib, ketoprofen, meloxicam, sulindac, or piroxicam 60 days prior) and patients hospitalized in the 21 days prior to and including the date of treatment initiation were excluded from this analysis.

Using RSS, patients treated with indomethacin were matched at a 1:1 ratio to controls randomly selected among patients treated with celecoxib, with direct matching on calendar date of treatment ( \pm 7 days), age ( \pm 5 years), sex, Charlson comorbidity index (exact) (92), time since confirmed COVID-19 ( \pm 5 days), and disease severity based on the highest-intensity COVID-19-related health service in the 7 days prior to and including the date of treatment initiation (lab service only vs. outpatient medical visit vs. emergency department visit) and symptom profile in the 21 days prior to and including the date of treatment initiation (recorded symptoms vs. none). This risk set sampled population was further matched on a propensity score (PS) (25) estimated using logistic regression with 24 demographic and clinical risk factors, including covariates related to baseline medical history and COVID-19 severity in the 21 days prior to treatment (table S11). Balance between indomethacin and celecoxib treatment groups was evaluated by comparison of absolute standardized differences in covariates, with an absolute standardized difference of less than 0.2 indicating good balance between the treatment groups (93).

The primary analysis was an intention-to-treat design, with follow-up beginning 1 day after indomethacin or celecoxib initiation and ending on the earliest occurrence of 30 days of follow-up reached or end of patient data. Odds ratios for the primary outcome of all-cause inpatient hospitalization were estimated for the RSS+PS matched population as well as for the RSS matched population. Our primary outcome definition required a record of inpatient hospital admission with a resulting inpatient stay; as a sensitivity, a broader outcome definition captured any hospital visit (defined with revenue and place of service codes).

\section{Observation of mechanical ventilation outcomes in in- patient new users of typical antipsychotics (treatment arm) vs. atypical antipsychotics (active comparator) using real-world data}

We used an incident user, active comparator design $(90,91)$ to assess the risk of mechanical ventilation among hospitalized COVID-19 patients treated with typical or atypical antipsychotics in an inpatient setting. See table S11 for a list of drugs included in each category. To permit assessment of 
day-level in-hospital confounders and outcomes, this analysis was restricted to hospitalized patients observable in hospital chargemaster data. Prevalent users of typical or atypical antipsychotics (any prescription fill or chargemaster-documented use in 60 days prior) and patients with evidence of mechanical ventilation in the 21 days prior to and including the date of treatment initiation were excluded from this analysis.

Using RSS, hospitalized patients treated with typical antipsychotics were matched at a 1:1 ratio to controls randomly selected among patients treated with atypical antipsychotics, with direct matching (1:1 fixed ratio) on calendar date of treatment ( \pm 7 days), age ( \pm 5 years), sex, Charlson comorbidity index (exact) (92), time since hospital admission, and disease severity as defined with a simplified version of the World Health Organization's ordinal scale for clinical improvement (94). This risk set sampled population was further matched on a PS estimated using logistic regression with 36 demographic and clinical risk factors, including covariates related to baseline medical history, admitting status, and disease severity at treatment (table S11). Balance between typical and atypical treatment groups was evaluated by comparison of absolute standardized differences in covariates, with an absolute standardized difference of less than 0.2 indicating good balance between the treatment groups (93).

The primary analysis was an intention-to-treat design, with follow-up beginning 1 day after the date of typical or atypical antipsychotic treatment initiation, and ending on the earliest occurrence of 30 days of follow-up reached, discharge from hospital, or end of patient data. Odds ratios for the primary outcome of inpatient mechanical ventilation were estimated for the RSS+PS matched population as well as for the RSS matched population.

\section{REFERENCES AND NOTES}

1. J. Liu, W. Xie, Y. Wang, Y. Xiong, S. Chen, J. Han, Q. Wu, A comparative overview of COVID-19, MERS and SARS: Review article. Int. J. Surg. 81, 1-8 (2020). doi:10.1016/j.ijsu.2020.07.032 Medline

2. J. H. Beigel, K. M. Tomashek, L. E. Dodd, A. K. Mehta, B. S. Zingman, A. C. Kalil, E. Hohmann, H. Y. Chu, A. Luetkemeyer, S. Kline, D. Lopez de Castilla, R. W. Finberg, K. Dierberg, V. Tapson, L. Hsieh, T. F. Patterson, R. Paredes, D. A. Sweeney, W. R. Short, G. Touloumi, D. C. Lye, N. Ohmagari, M.-d. Oh, G. M. Ruiz-Palacios, T. Benfield, G. Fätkenheuer, M. G. Kortepeter, R. L. Atmar, C. B. Creech, J. Lundgren, A. G. Babiker, S. Pett, J. D. Neaton, T. H. Burgess, T. Bonnett, M. Green, M. Makowski, A. Osinusi, S. Nayak, H. C. Lane, ACTT-1 Study Group Members, Remdesivir for the treatment of Covid-19-Final report. N. Engl. J. Med. 10.1056/NEJMoa2007764 (2020). doi:10.1056/NEJMoa2007764

3. RECOVERY Collaborative Group, Dexamethasone in Hospitalized Patients with Covid-19 - Preliminary Report. N. Engl. J. Med. 10.1056/nejmoa2021436 (2020). doi:10.1056/nejmoa2021436 Medline

4. M. Becerra-Flores, T. Cardozo, SARS-CoV-2 viral spike G614 mutation exhibits higher case fatality rate. Int. J. Clin. Pract. 74, el3525 (2020). doi:10.1111/ijcp.13525 Medline

5. D. E. Gordon, G. M. Jang, M. Bouhaddou, J. Xu, K. Obernier, K. M. White, M. J. O’Meara, V. V. Rezelj, J. Z. Guo, D. L. Swaney, T. A. Tummino, R. Hüttenhain, R. M. Kaake, A. L. Richards, B. Tutuncuoglu, H. Foussard, J. Batra, K. Haas, M. Modak, M. Kim, P. Haas, B. J. Polacco, H. Braberg, J. M. Fabius, M. Eckhardt, M.
Soucheray, M. J. Bennett, M. Cakir, M. J. McGregor, Q. Li, B. Meyer, F. Roesch, T. Vallet, A. Mac Kain, L. Miorin, E. Moreno, Z. Z. C. Naing, Y. Zhou, S. Peng, Y. Shi, Z. Zhang, W. Shen, I. T. Kirby, J. E. Melnyk, J. S. Chorba, K. Lou, S. A. Dai, I. BarrioHernandez, D. Memon, C. Hernandez-Armenta, J. Lyu, C. J. P. Mathy, T. Perica, K. B. Pilla, S. J. Ganesan, D. J. Saltzberg, R. Rakesh, X. Liu, S. B. Rosenthal, L. Calviello, S. Venkataramanan, J. Liboy-Lugo, Y. Lin, X.-P. Huang, Y. Liu, S. A. Wankowicz, M. Bohn, M. Safari, F. S. Ugur, C. Koh, N. S. Savar, Q. D. Tran, D. Shengjuler, S. J. Fletcher, M. C. O'Neal, Y. Cai, J. C. J. Chang, D. J. Broadhurst, S. Klippsten, P. P. Sharp, N. A. Wenzell, D. Kuzuoglu-Ozturk, H.-Y. Wang, R. Trenker, J. M. Young, D. A. Cavero, J. Hiatt, T. L. Roth, U. Rathore, A. Subramanian, J. Noack, M. Hubert, R. M. Stroud, A. D. Frankel, O. S. Rosenberg, K. A. Verba, D. A. Agard, M. Ott, M. Emerman, N. Jura, M. von Zastrow, E. Verdin, A. Ashworth, 0. Schwartz, C. d'Enfert, S. Mukherjee, M. Jacobson, H. S. Malik, D. G. Fujimori, T. Ideker, C. S. Craik, S. N. Floor, J. S. Fraser, J. D. Gross, A. Sali, B. L. Roth, D. Ruggero, J. Taunton, T. Kortemme, P. Beltrao, M. Vignuzzi, A. García-Sastre, K. M. Shokat, B. K. Shoichet, N. J. Krogan, A SARS-CoV-2 protein interaction map reveals targets for drug repurposing. Nature 583, 459-468 (2020). doi:10.1038/s41586-020-2286-9 Medline

6. G. Teo, G. Liu, J. Zhang, A. I. Nesvizhskii, A.-C. Gingras, H. Choi, SAINTexpress: Improvements and additional features in Significance Analysis of INTeractome software. J. Proteomics 100, 37-43 (2014). doi:10.1016/j.jprot.2013.10.023 Medline

7. S. Jäger, P. Cimermancic, N. Gulbahce, J. R. Johnson, K. E. McGovern, S. C. Clarke, M. Shales, G. Mercenne, L. Pache, K. Li, H. Hernandez, G. M. Jang, S. L. Roth, E. Akiva, J. Marlett, M. Stephens, I. D’Orso, J. Fernandes, M. Fahey, C. Mahon, A. J. O'Donoghue, A. Todorovic, J. H. Morris, D. A. Maltby, T. Alber, G. Cagney, F. D. Bushman, J. A. Young, S. K. Chanda, W. I. Sundquist, T. Kortemme, R. D. Hernandez, C. S. Craik, A. Burlingame, A. Sali, A. D. Frankel, N. J. Krogan, Global landscape of HIV-human protein complexes. Nature 481, 365-370 (2011). doi:10.1038/nature10719 Medline

8. J. C. Young, N. J. Hoogenraad, F. U. Hartl, Molecular chaperones Hsp90 and Hsp70 deliver preproteins to the mitochondrial import receptor Tom70. Cell 112, 41-50 (2003). doi:10.1016/S0092-8674(02)01250-3 Medline

9. R. Lin, S. Paz, J. Hiscott, Tom70 imports antiviral immunity to the mitochondria. Cell Res. 20, 971-973 (2010). doi:10.1038/cr.2010.113 Medline

10. B. Wei, Y. Cui, Y. Huang, H. Liu, L. Li, M. Li, K.-C. Ruan, Q. Zhou, C. Wang, Tom70 mediates Sendai virus-induced apoptosis on mitochondria. J. Virol. 89, 38043818 (2015). doi:10.1128/JVL.02959-14 Medline

11. A. M. Edmonson, D. K. Mayfield, V. Vervoort, B. R. DuPont, G. Argyropoulos, Characterization of a human import component of the mitochondrial outer membrane, TOMM70A. Cell Commun. Adhes. 9, 15-27 (2002). doi:10.1080/15419060212186 Medline

12. M. J. Baker, A. E. Frazier, J. M. Gulbis, M. T. Ryan, Mitochondrial protein-import machinery: Correlating structure with function. Trends Cell Biol. 17, 456-464 (2007). doi:10.1016/i.tcb.2007.07.010 Medline

13. J. Brix, K. Dietmeier, N. Pfanner, Differential recognition of preproteins by the purified cytosolic domains of the mitochondrial import receptors Tom20, Tom22, and Tom70. J. Biol. Chem. 272, 20730-20735 (1997). doi:10.1074/jbc.272.33.20730 Medline

14. J. Brix, G. A. Ziegler, K. Dietmeier, J. Schneider-Mergener, G. E. Schulz, N. Pfanner, The mitochondrial import receptor Tom70: Identification of a $25 \mathrm{kDa}$ core domain with a specific binding site for preproteins. J. Mol. Biol. 303, 479-488 (2000). doi:10.1006/imbi.2000.4120 Medline

15. R. D. Mills, J. Trewhella, T. W. Qiu, T. Welte, T. M. Ryan, T. Hanley, R. B. Knott, T. Lithgow, T. D. Mulhern, Domain organization of the monomeric form of the Tom70 mitochondrial import receptor. J. Mol. Biol. 388, 1043-1058 (2009). doi:10.1016/i.jmb.2009.03.070 Medline

16. S. D. Weeks, S. De Graef, A. Munawar, X-ray Crystallographic Structure of Orf9b from SARS-CoV-2 (2020); https://doi.org/10.2210/pdb6z4u/pdb.

17. M. Bouhaddou, D. Memon, B. Meyer, K. M. White, V. V. Rezelj, M. Correa Marrero, B. J. Polacco, J. E. Melnyk, S. Ulferts, R. M. Kaake, J. Batra, A. L. Richards, E. Stevenson, D. E. Gordon, A. Rojc, K. Obernier, J. M. Fabius, M. Soucheray, L. Miorin, E. Moreno, C. Koh, Q. D. Tran, A. Hardy, R. Robinot, T. Vallet, B. E. NilssonPayant, C. Hernandez-Armenta, A. Dunham, S. Weigang, J. Knerr, M. Modak, D. Quintero, Y. Zhou, A. Dugourd, A. Valdeolivas, T. Patil, Q. Li, R. Hüttenhain, M. 
Cakir, M. Muralidharan, M. Kim, G. Jang, B. Tutuncuoglu, J. Hiatt, J. Z. Guo, J. Xu, S. Bouhaddou, C. J. P. Mathy, A. Gaulton, E. J. Manners, E. Félix, Y. Shi, M. Goff, J. K. Lim, T. McBride, M. C. O'Neal, Y. Cai, J. C. J. Chang, D. J. Broadhurst, S. Klippsten, E. De Wit, A. R. Leach, T. Kortemme, B. Shoichet, M. Ott, J. SaezRodriguez, B. R. tenOever, R. D. Mullins, E. R. Fischer, G. Kochs, R. Grosse, A. García-Sastre, M. Vignuzzi, J. R. Johnson, K. M. Shokat, D. L. Swaney, P. Beltrao, N. J. Krogan, The Global Phosphorylation Landscape of SARS-CoV-2 Infection. Cell 182, 685-712.e19 (2020). doi:10.1016/i.cell.2020.06.034 Medline

18. J. Li, X. Qian, J. Hu, B. Sha, Molecular chaperone Hsp70/Hsp90 prepares the mitochondrial outer membrane translocon receptor Tom71 for preprotein loading. J. Biol. Chem. 284, 23852-23859 (2009). doi:10.1074/jbc.M109.023986 Medline

19. X.-Y. Liu, B. Wei, H.-X. Shi, Y.-F. Shan, C. Wang, Tom70 mediates activation of interferon regulatory factor 3 on mitochondria. Cell Res. 20, 994-1011 (2010). doi:10.1038/cr.2010.103 Medline

20. Y. Liu, C. Zhang, F. Huang, Y. Yang, F. Wang, J. Yuan, Z. Zhang, Y. Qin, X. Li, D. Zhao, S. Li, S. Tan, Z. Wang, J. Li, C. Shen, J. Li, L. Peng, W. Wu, M. Cao, L. Xing, Z. Xu, L. Chen, C. Zhou, W. J. Liu, L. Liu, C. Jiang, Elevated plasma levels of selective cytokines in COVID-19 patients reflect viral load and lung injury. Natl. Sci. Rev. 7 , 1003-1011 (2020). doi:10.1093/nsr/nwaa037

21. C. Huang, Y. Wang, X. Li, L. Ren, J. Zhao, Y. Hu, L. Zhang, G. Fan, J. Xu, X. Gu, Z. Cheng, T. Yu, J. Xia, Y. Wei, W. Wu, X. Xie, W. Yin, H. Li, M. Liu, Y. Xiao, H. Gao, L. Guo, J. Xie, G. Wang, R. Jiang, Z. Gao, Q. Jin, J. Wang, B. Cao, Clinical features of patients infected with 2019 novel coronavirus in Wuhan, China. Lancet 395, 497506 (2020). doi:10.1016/S0140-6736(20)30183-5 Medline

22. C. Qin, L. Zhou, Z. Hu, S. Zhang, S. Yang, Y. Tao, C. Xie, K. Ma, K. Shang, W. Wang, D.-S. Tian, Dysregulation of Immune Response in Patients With Coronavirus 2019 (COVID-19) in Wuhan, China. Clin. Infect. Dis. 71, 762-768 (2020). doi:10.1093/cid/ciaa248 Medline

23. G. Chen, D. Wu, W. Guo, Y. Cao, D. Huang, H. Wang, T. Wang, X. Zhang, H. Chen, H. Yu, X. Zhang, M. Zhang, S. Wu, J. Song, T. Chen, M. Han, S. Li, X. Luo, J. Zhao, Q. Ning, Clinical and immunological features of severe and moderate coronavirus disease 2019. J. Clin. Invest. 130, 2620-2629 (2020). doi:10.1172/JC|137244 Medline

24. C. Amici, A. Di Caro, A. Ciucci, L. Chiappa, C. Castilletti, V. Martella, N. Decaro, C. Buonavoglia, M. R. Capobianchi, M. G. Santoro, Indomethacin has a potent antiviral activity against SARS coronavirus. Antivir. Ther. 11, 1021-1030 (2006). Medline

25. P. R. Rosenbaum, D. B. Rubin, The central role of the propensity score in observational studies for causal effects. Biometrika 70, 41-55 (1983). doi:10.1093/biomet/70.1.41

26. C. Abate, P. D. Mosier, F. Berardi, R. A. Glennon, A structure-affinity and comparative molecular field analysis of sigma-2 ( $\circ 2)$ receptor ligands. Cent. Nerv. Syst. Agents Med. Chem. 9, 246-257 (2009). doi:10.2174/1871524910909030246 Medline

27. R. A. Glennon, Sigma receptor ligands and the use thereof, U.S. Patent $6,057,371$ (2000);

https://patentimages.storage.googleapis.com/dc/36/68/73f4ccdac4c973/US 6057371.pdf.

28. R. R. Matsumoto, B. Pouw, Correlation between neuroleptic binding to $\sigma_{1}$ and $\sigma_{2}$ receptors and acute dystonic reactions. Eur. J. Pharmacol. 401, 155-160 (2000). doi:10.1016/S0014-2999(00)00430-1 Medline

29. M. Dold, M. T. Samara, C. Li, M. Tardy, S. Leucht, Haloperidol versus firstgeneration antipsychotics for the treatment of schizophrenia and other psychotic disorders. Cochrane Database Syst. Rev. 1, CD009831 (2015). doi:10.1002/14651858.CD009831.pub2 Medline

30. F. F. Moebius, R. J. Reiter, K. Bermoser, H. Glossmann, S. Y. Cho, Y. K. Paik, Pharmacological analysis of sterol delta8-delta7 isomerase proteins with [3H]ifenprodil. Mol. Pharmacol. 54, 591-598 (1998). doi:10.1124/mol.54.3.591 Medline

31. E. Gregori-Puigjané, V. Setola, J. Hert, B. A. Crews, J. J. Irwin, E. Lounkine, L. Marnett, B. L. Roth, B. K. Shoichet, Identifying mechanism-of-action targets for drugs and probes. Proc. Natl. Acad. Sci. U.S.A. 109, 11178-11183 (2012). doi:10.1073/pnas.1204524109 Medline

32. Z. Hubler, D. Allimuthu, I. Bederman, M. S. Elitt, M. Madhavan, K. C. Allan, H. E.
Shick, E. Garrison, M. T Karl, D. C. Factor, Z. S. Nevin, J. L. Sax, M. A. Thompson, Y. Fedorov, J. Jin, W. K. Wilson, M. Giera, F. Bracher, R. H. Miller, P. J. Tesar, D. J. Adams, Accumulation of 8,9-unsaturated sterols drives oligodendrocyte formation and remyelination. Nature 560, 372-376 (2018). doi:10.1038/s41586018-0360-3 Medline

33. F. F. Moebius, R. J. Reiter, M. Hanner, H. Glossmann, High affinity of sigma 1binding sites for sterol isomerization inhibitors: Evidence for a pharmacological relationship with the yeast sterol C8-C7 isomerase. Br. J. Pharmacol. 121, 1-6 (1997). doi:10.1038/sj.bjp.0701079 Medline

34. H.-W. Jiang, H.-N. Zhang, Q.-F. Meng, J. Xie, Y. Li, H. Chen, Y.-X. Zheng, X.-N. Wang, H. Qi, J. Zhang, P.-H. Wang, Z.-G. Han, S.-C. Tao, SARS-CoV-2 Orf9b suppresses type I interferon responses by targeting TOM70. Cell. Mol. Immunol. 17, 998-1000 (2020). doi:10.1038/s41423-020-0514-8 Medline

35. The COVID-19 Host Genetics Initiative, The COVID-19 Host Genetics Initiative, a global initiative to elucidate the role of host genetic factors in susceptibility and severity of the SARS-CoV-2 virus pandemic. Eur. J. Hum. Genet. 28, 715-718 (2020). doi:10.1038/s41431-020-0636-6 Medline

36. J. J. Almagro Armenteros, C. K. Sønderby, S. K. Sønderby, H. Nielsen, O. Winther, DeepLoc: Prediction of protein subcellular localization using deep learning. Bioinformatics 33, 3387-3395 (2017). doi:10.1093/bioinformatics/btx431 Medline

37. C. Chiva, R. Olivella, E. Borràs, G. Espadas, O. Pastor, A. Solé, E. Sabidó, QCloud: A cloud-based quality control system for mass spectrometry-based proteomics laboratories. PLOS ONE 13, e0189209 (2018). doi:10.1371/journal.pone.0189209 Medline

38. J. Cox, M. Mann, MaxQuant enables high peptide identification rates, individualized p.p.b.-range mass accuracies and proteome-wide protein quantification. Nat. Biotechnol. 26, 1367-1372 (2008). doi:10.1038/nbt.1511 Medline

39. M. Giurgiu, J. Reinhard, B. Brauner, I. Dunger-Kaltenbach, G. Fobo, G. Frishman, C. Montrone, A. Ruepp, CORUM: The comprehensive resource of mammalian protein complexes-2019. Nucleic Acids Res. 47, D559-D563 (2019). doi:10.1093/nar/gky973 Medline

40. E. L. Huttlin, L. Ting, R. J. Bruckner, F. Gebreab, M. P. Gygi, J. Szpyt, S. Tam, G. Zarraga, G. Colby, K. Baltier, R. Dong, V. Guarani, L. P. Vaites, A. Ordureau, R. Rad, B. K. Erickson, M. Wühr, J. Chick, B. Zhai, D. Kolippakkam, J. Mintseris, R. A. Obar T. Harris, S. Artavanis-Tsakonas, M. E. Sowa, P. De Camilli, J. A. Paulo, J. W. Harper, S. P. Gygi, The BioPlex Network: A Systematic Exploration of the Human Interactome. Cell 162, 425-440 (2015). doi:10.1016/i.cell.2015.06.043 Medline

41. G. Yu, L.-G. Wang, Y. Han, Q.-Y. He, clusterProfiler: An R package for comparing biological themes among gene clusters. OMICS 16, 284-287 (2012). doi:10.1089/omi.2011.0118 Medline

42. M. Remmert, A. Biegert, A. Hauser, J. Söding, HHblits: Lightning-fast iterative protein sequence searching by HMM-HMM alignment. Nat. Methods 9, 173-175 (2011). doi:10.1038/nmeth.1818 Medline

43. J. Yang, I. Anishchenko, H. Park, Z. Peng, S. Ovchinnikov, D. Baker, Improved protein structure prediction using predicted interresidue orientations. Proc. Natl. Acad. Sci. U.S.A. 117, 1496-1503 (2020). doi:10.1073/pnas.1914677117 Medline

44. Y. Zhai, F. Sun, X. Li, H. Pang, X. Xu, M. Bartlam, Z. Rao, Insights into SARS-CoV transcription and replication from the structure of the nsp7-nsp8 hexadecamer. Nat. Struct. Mol. Biol. 12, 980-986 (2005). doi:10.1038/nsmb999 Medline

45. A. Waterhouse, M. Bertoni, S. Bienert, G. Studer, G. Tauriello, R. Gumienny, F. T. Heer, T. A. P. de Beer, C. Rempfer, L. Bordoli, R. Lepore, T. Schwede, SWISSMODEL: Homology modelling of protein structures and complexes. Nucleic Acids Res. 46, W296-W303 (2018). doi:10.1093/nar/gky427 Medline

46. J. Durairaj, M. Akdel, D. de Ridder, A. D. J. van Dijk, Geometricus Represents Protein Structures as Shape-mers Derived from Moment Invariants. bioRxiv 2020.09.07.285569 [Preprint]. 8 September 2020. https://doi.org/10.1101/2020.09.07.285569.

47. M. Akdel, J. Durairaj, D. de Ridder, A. D. J. van Dijk, Caretta - A multiple protein structure alignment and feature extraction suite. Comput. Struct. Biotechnol. J. 18, 981-992 (2020). doi:10.1016/i.csbj.2020.03.011 Medline

48. P. Shannon, A. Markiel, O. Ozier, N. S. Baliga, J. T. Wang, D. Ramage, N. Amin, B. Schwikowski, T. Ideker, Cytoscape: A software environment for integrated models of biomolecular interaction networks. Genome Res. 13, 2498-2504 (2003). 
doi:10.1101/gr.1239303 Medline

49. R. T. Pillich, J. Chen, V. Rynkov, D. Welker, D. Pratt, NDEx: A Community Resource for Sharing and Publishing of Biological Networks. Methods Mol. Biol. 1558, 271301 (2017). doi:10.1007/978-1-4939-6783-4 13 Medline

50. D. K. W. Chu, Y. Pan, S. M. S. Cheng, K. P. Y. Hui, P. Krishnan, Y. Liu, D. Y. M. Ng, C. K. C. Wan, P. Yang, Q. Wang, M. Peiris, L. L. M. Poon, Molecular Diagnosis of a Novel Coronavirus (2019-nCoV) Causing an Outbreak of Pneumonia. Clin. Chem. 66, 549-555 (2020). doi:10.1093/clinchem/hvaa029 Medline

51. K. J. Livak, T. D. Schmittgen, Analysis of relative gene expression data using realtime quantitative PCR and the 2(-Delta Delta C(T)) Method. Methods 25, 402-408 (2001). doi:10.1006/meth.2001.1262 Medline

52. R. Stoner, T. Maures, D. Conant, Methods and systems for guide RNA design and use, U.S. Patent 2019/0382797 A1 (2019); https://patentimages. storage.googleapis.com/95/c7/43/3d48387ce0f116/US 20190382797A1.pdf.

53. T. Hsiau, D. Conant, N. Rossi, T. Maures, K. Waite, J. Yang, S. Joshi, R. Kelso, K. Holden, B. L. Enzmann, R. Stoner, Inference of CRISPR Edits from Sanger Trace Data. bioRxiv 251082 [Preprint]. 10 August 2018. https://doi.org/10.1101/251082.

54. A. S. Jureka, J. A. Silvas, C. F. Basler, Propagation, Inactivation, and Safety Testing of SARS-CoV-2. Viruses 12, 622 (2020). doi:10.3390/v12060622 Medline

55. A. C. Y. Fan, M. K. Bhangoo, J. C. Young, Hsp90 functions in the targeting and outer membrane translocation steps of Tom70-mediated mitochondrial import. J. Biol. Chem. 281, 33313-33324 (2006). doi:10.1074/jbc.M605250200 Medline

56. S. Backes, S. Hess, F. Boos, M. W. Woellhaf, S. Gödel, M. Jung, T. Mühlhaus, J. M. Herrmann, Tom70 enhances mitochondrial preprotein import efficiency by binding to internal targeting sequences. J. Cell Biol. 217, 1369-1382 (2018). doi:10.1083/jcb.201708044 Medline

57. J. J. Almagro Armenteros, M. Salvatore, O. Emanuelsson, O. Winther, G. von Heijne, A. Elofsson, H. Nielsen, Detecting sequence signals in targeting peptides using deep learning. Life Sci. Alliance 2, e201900429 (2019). doi:10.26508/Isa.201900429 Medline

58. Y. Fukasawa, J. Tsuji, S.-C. Fu, K. Tomii, P. Horton, K. Imai, MitoFates: Improved prediction of mitochondrial targeting sequences and their cleavage sites. Mol. Cell. Proteomics 14, 1113-1126 (2015). doi:10.1074/mcp.M114.043083 Medline

59. A. Drozdetskiy, C. Cole, J. Procter, G. J. Barton, JPred4: A protein secondary structure prediction server. Nucleic Acids Res. 43, W389-W394 (2015). doi:10.1093/nar/gkv332 Medline

60. D. N. Mastronarde, Automated electron microscope tomography using robust prediction of specimen movements. J. Struct. Biol. 152, 36-51 (2005). doi:10.1016/i.jsb.2005.07.007 Medline

61. S. Q. Zheng, E. Palovcak, J.-P. Armache, K. A. Verba, Y. Cheng, D. A. Agard, MotionCor2: Anisotropic correction of beam-induced motion for improved cryoelectron microscopy. Nat. Methods 14, 331-332 (2017). doi:10.1038/nmeth.4193 Medline

62. S. F. Altschul, T. L. Madden, A. A. Schäffer, J. Zhang, Z. Zhang, W. Miller, D. J. Lipman, Gapped BLAST and PSI-BLAST: A new generation of protein database search programs. Nucleic Acids Res. 25, 3389-3402 (1997). doi:10.1093/nar/25.17.3389 Medline

63. P. Emsley, K. Cowtan, Coot: Model-building tools for molecular graphics. Acta Cryst. D60, 2126-2132 (2004). doi:10.1107/S0907444904019158 Medline

64. R. Y.-R. Wang, Y. Song, B. A. Barad, Y. Cheng, J. S. Fraser, F. DiMaio, Automated structure refinement of macromolecular assemblies from cryo-EM maps using Rosetta. eLife 5, e17219 (2016). doi:10.7554/eLife.17219 Medline

65. R. T. Kidmose, J. Juhl, P. Nissen, T. Boesen, J. L. Karlsen, B. P. Pedersen, Namdinator - automatic molecular dynamics flexible fitting of structural models into cryo-EM and crystallography experimental maps. IUCrJ 6, 526-531 (2019). doi:10.1107/S2052252519007619 Medline

66. T. I. Croll, ISOLDE: A physically realistic environment for model building into lowresolution electron-density maps. Acta Cryst. D74, 519-530 (2018). doi:10.1107/S2059798318002425 Medline

67. T. D. Goddard, C. C. Huang, E. C. Meng, E. F. Pettersen, G. S. Couch, J. H. Morris, T. E. Ferrin, UCSF ChimeraX: Meeting modern challenges in visualization and analysis. Protein Sci. 27, 14-25 (2018). doi:10.1002/pro.3235 Medline

68. P. V. Afonine, B. P. Klaholz, N. W. Moriarty, B. K. Poon, O. V. Sobolev, T. C.
Terwilliger, P. D. Adams, A. Urzhumtsev, New tools for the analysis and validation of cryo-EM maps and atomic models. Acta Cryst. D74, 814-840 (2018). doi:10.1107/S2059798318009324 Medline

69. E. Krissinel, K. Henrick, Inference of macromolecular assemblies from crystalline state. J. Mol. Biol. 372, 774-797 (2007). doi:10.1016/j.jmb.2007.05.022 Medline

70. B. B. Sun, J. C. Maranville, J. E. Peters, D. Stacey, J. R. Staley, J. Blackshaw, S. Burgess, T. Jiang, E. Paige, P. Surendran, C. Oliver-Williams, M. A. Kamat, B. P. Prins, S. K. Wilcox, E. S. Zimmerman, A. Chi, N. Bansal, S. L. Spain, A. M. Wood, N. W. Morrell, J. R. Bradley, N. Janjic, D. J. Roberts, W. H. Ouwehand, J. A. Todd, N. Soranzo, K. Suhre, D. S. Paul, C. S. Fox, R. M. Plenge, J. Danesh, H. Runz, A. S. Butterworth, Genomic atlas of the human plasma proteome. Nature 558, 73-79 (2018). doi:10.1038/s41586-018-0175-2 Medline

71. Z. Zhu, Z. Zheng, F. Zhang, Y. Wu, M. Trzaskowski, R. Maier, M. R. Robinson, J. J. McGrath, P. M. Visscher, N. R. Wray, J. Yang, Causal associations between risk factors and common diseases inferred from GWAS summary data. Nat. Commun. 9, 224 (2018). doi:10.1038/s41467-017-02317-2 Medline

72. A. N. Honko, N. Storm, D. J. Bean, J. H. Vasquez, S. N. Downs, A. Griffiths, Rapid Quantification and Neutralization Assays for Novel Coronavirus SARS-CoV-2 Using Avicel RC-591 Semi-Solid Overlay. Preprints 2020050264 [Preprint]. 16 May 2020. www.preprints.org/manuscript/202005.0264/v1.

73. A. Sali, T. L. Blundell, Comparative protein modelling by satisfaction of spatial restraints. J. Mol. Biol. 234, 779-815 (1993). doi:10.1006/imbi.1993.1626 Medline

74. T. Yamada, J. Komoto, K. Watanabe, Y. Ohmiya, F. Takusagawa, Crystal structure and possible catalytic mechanism of microsomal prostaglandin E synthase type 2 (mPGES-2). J. Mol. Biol. 348, 1163-1176 (2005). doi:10.1016/i.jmb.2005.03.035 Medline

75. W. Yin, C. Mao, X. Luan, D.-D. Shen, Q. Shen, H. Su, X. Wang, F. Zhou, W. Zhao, M. Gao, S. Chang, Y.-C. Xie, G. Tian, H.-W. Jiang, S.-C. Tao, J. Shen, Y. Jiang, H. Jiang, Y. Xu, S. Zhang, Y. Zhang, H. E. Xu, Structural basis for inhibition of the RNAdependent RNA polymerase from SARS-CoV-2 by remdesivir. Science 368, 1499 1504 (2020). doi:10.1126/science.abc1560 Medline

76. D. Kozakov, D. R. Hall, B. Xia, K. A. Porter, D. Padhorny, C. Yueh, D. Beglov, S. Vajda, The ClusPro web server for protein-protein docking. Nat. Protoc. 12, 255-278 (2017). doi:10.1038/nprot.2016.169 Medline

77. B. G. Pierce, K. Wiehe, H. Hwang, B.-H. Kim, T. Vreven, Z. Weng, ZDOCK server: Interactive docking prediction of protein-protein complexes and symmetric multimers. Bioinformatics 30, 1771-1773 (2014). doi:10.1093/bioinformatics/btu097 Medline

78. Y. Yan, H. Tao, J. He, S.-Y. Huang, The HDOCK server for integrated protein-protein docking. Nat. Protoc. 15, 1829-1852 (2020). doi:10.1038/s41596-020-0312-x Medline

79. A. Tovchigrechko, I. A. Vakser, GRAMM-X public web server for protein-protein docking. Nucleic Acids Res. 34, W310-W314 (2006). doi:10.1093/nar/gkl206 Medline

80. M. Torchala, I. H. Moal, R. A. G. Chaleil, J. Fernandez-Recio, P. A. Bates, SwarmDock: A server for flexible protein-protein docking. Bioinformatics 29, 807-809 (2013). doi:10.1093/bioinformatics/btt038 Medline

81. D. Schneidman-Duhovny, Y. Inbar, R. Nussinov, H. J. Wolfson, PatchDock and SymmDock: Servers for rigid and symmetric docking. Nucleic Acids Res. 33, W363-W367 (2005). doi:10.1093/nar/gki481 Medline

82. G. Q. Dong, H. Fan, D. Schneidman-Duhovny, B. Webb, A. Sali, Optimized atomic statistical potentials: Assessment of protein interfaces and loops. Bioinformatics 29, 3158-3166 (2013). doi:10.1093/bioinformatics/btt560 Medline

83. J. Armstrong, G. Hickey, M. Diekhans, A. Deran, Q. Fang, D. Xie, S. Feng, J. Stiller, D. Genereux, J. Johnson, V. D. Marinescu, D. Haussler, J. Alföldi, K. Lindblad-Toh, E. Karlsson, E. D. Jarvis, G. Zhang, B. Paten, Progressive alignment with Cactus: A multiple-genome aligner for the thousand-genome era. bioRxiv 730531 [Preprint]. 15 October 2019. https://doi.org/10.1101/730531.

84. B. Paten, D. Earl, N. Nguyen, M. Diekhans, D. Zerbino, D. Haussler, Cactus: Algorithms for genome multiple sequence alignment. Genome Res. 21, 1512-1528 (2011). doi:10.1101/gr.123356.111 Medline

85. M. D. Smith, J. O. Wertheim, S. Weaver, B. Murrell, K. Scheffler, S. L. Kosakovsky Pond, Less is more: An adaptive branch-site random effects model for efficient detection of episodic diversifying selection. Mol. Biol. Evol. 32, 1342-1353 (2015). doi:10.1093/molbev/msv022 Medline 
86. S. L. K. Pond, S. D. W. Frost, S. V. Muse, HyPhy: Hypothesis testing using $\begin{array}{llll}\text { phylogenies. } & \text { Bioinformatics } 21, & 676-679 & \text { (2005). }\end{array}$ doi:10.1093/bioinformatics/bti079 Medline

87. K. S. Pollard, M. J. Hubisz, K. R. Rosenbloom, A. Siepel, Detection of nonneutral substitution rates on mammalian phylogenies. Genome Res. 20, 110-121 (2010). doi:10.1101/gr.097857.109 Medline

88. M. J. Hubisz, K. S. Pollard, A. Siepel, PHAST and RPHAST: Phylogenetic analysis with space/time models. Brief. Bioinform. 12, 41-51 (2011). doi:10.1093/bib/bbq072 Medline

89. R. Ramani, K. Krumholz, Y.-F. Huang, A. Siepel, PhastWeb: A web interface for evolutionary conservation scoring of multiple sequence alignments using phastCons and phyloP. Bioinformatics 35, 2320-2322 (2019). doi:10.1093/bioinformatics/bty966 Medline

90. W. A. Ray, Evaluating medication effects outside of clinical trials: New-user designs. Am. J. Epidemiol. 158, 915-920 (2003). doi:10.1093/aje/kwg231 Medline

91. S. Schneeweiss, A basic study design for expedited safety signal evaluation based on electronic healthcare data. Pharmacoepidemiol. Drug Saf. 19, 858-868 (2010). doi:10.1002/pds.1926 Medline

92. H. Quan, V. Sundararajan, P. Halfon, A. Fong, B. Burnand, J.-C. Luthi, L. D. Saunders, C. A. Beck, T. E. Feasby, W. A. Ghali, Coding algorithms for defining comorbidities in ICD-9-CM and ICD-10 administrative data. Med. Care 43, 11301139 (2005). doi:10.1097/01.mlr.0000182534.19832.83 Medline

93. P. C. Austin, Balance diagnostics for comparing the distribution of baseline covariates between treatment groups in propensity-score matched samples. Stat. Med. 28, 3083-3107 (2009). doi:10.1002/sim.3697 Medline

94. World Health Organization (WHO), WHO R\&D Blueprint, novel Coronavirus: COVID-19 Therapeutic Trial Synopsis (WHO, 2020); www.who.int/blueprint/priority-diseases/key-action/COVID19 Treatment Trial Design Master Protocol synopsis Final 18022020.pdf.

95. Y. Perez-Riverol, A. Csordas, J. Bai, M. Bernal-Llinares, S. Hewapathirana, D. J. Kundu, A. Inuganti, J. Griss, G. Mayer, M. Eisenacher, E. Pérez, J. Uszkoreit, J. Pfeuffer, T. Sachsenberg, S. Yilmaz, S. Tiwary, J. Cox, E. Audain, M. Walzer, A. F. Jarnuczak, T. Ternent, A. Brazma, J. A. Vizcaíno, The PRIDE database and related tools and resources in 2019: Improving support for quantification data. Nucleic Acids Res. 47, D442-D450 (2019). doi:10.1093/nar/gky1106 Medline

96. J. Li, X. Qian, J. Hu, B. Sha, Crystal structure of Tom71 complexed with Hsp82 Cterminal fragment (2009); https://doi.org/10.2210/pdb3fp2/pdb.

\section{ACKNOWLEDGMENTS}

The authors acknowledge their partners and families for support in child care and other matters during this time. The views, opinions, and findings contained in this study are those of the authors and do not represent the official views, policies, or endorsement of the Department of Defense or the U.S. Government. Imaging at the University of Sheffield was performed in the Wolfson Light Microscopy facility. A549 cells stably expressing ACE2 (A549-ACE2) were kindly provided by Dr. Olivier Schwartz. We thank Harmit Malik for helpful discussion. We thank Mehmet Akdel and Janani Durairaj for input on analysis, Randy Albrecht for support with the BSL3 facility and procedures at the ISMMS, and Richard Cadagan for technical assistance. We thank the High Containment Core at Georgia State University for supporting the BSL3 facility and procedures. We thank Assaf Alon and Andrew C. Kruse for helpful discussions on sigma receptor biology. We thank Drs. Steven Deeks, Peter J. Hunt, John Gordan, Chiara Corbetta-Rastelli and Emma Lantos for insight on clinical applications for drug repurposing. Funding: This research was funded by grants from the National Institutes of Health, NIH (P01AI063302, P50Al150476, R01Al120694, R01Al122747, R01Al143292, U19AI135972 and U19Al135990 to N.J.K.; P01Al120943, R01Al143292 to C.F.B.; U19 Al135990 to T.I.); by National Institute of Allergy and Infectious Diseases R01Al128214 to O.R.; National Institute of Neurological Disorders and Stroke R01 NS089713, the NIH Office of the Director Al150476 and NIGMS R01 GM24485 to R.S.; by a Fast Grant for COVID-19 from the Emergent Ventures program at the Mercatus Center of George Mason University (N.J.K.) and a separate Fast Grant for COVID-19 (C.F.B); by Roddenberry Foundation Gladstone Institutes to K.S.P.; from the Defense Advanced Research Projects Agency (HR0011-19-2-0020 to B.K.S., N.J.K.,
K.A.V., D.A.A., A.G.-S. and K.M.S.; HRO011-20-2-0040 to M.B.F.); NIGMS R35GM122481 (to B.K.S.); by CRIP (Center for Research for Influenza Pathogenesis), a NIAID supported Center of Excellence for Influenza Research and Surveillance (CEIRS, contract \# HHSN272201400008C) to A.G.-S.; by supplements to NIAID grant U19Al135972 and DoD grant W81XWH-20-1-0270 to A.G.-S.; by the Bill and Melinda Gates Foundation (INV-006099) and BARDA (ASPR-20-01495) to M.B.F., by Howard Hughes Medical Institute to K.S.; by Damon Runyon Cancer Research Foundation DRG-2402-20 to C.P.; by Burroughs Wellcome Fund 1019894 to N.H.; by the Chan Zuckerberg Initiative to O.S. and T.K.; by Cytoscape: A modeling platform for biomolecular networks (NHGRI R01 HG009979) to T.I.; by the generous support of the JPB Foundation, the Open Philanthropy Project (research grant 2020-215611 (5384) and by anonymous donors to A.G.-S.; a Laboratoire d'Excellence grant ANR-10-LABX62-IBEID and the URGENCE COVID-19 Institut Pasteur fundraising campaign to M.V and N.J.; by grants from the BBSRC (BB/S009566/1 and BB/L002841/1) to A.P. and D.W. and by BBSRC White Rose DTP (BB/J014443/1) to A.S-S.; by The Augusta University-Georgia State University Seed Grant program to C.F.B.; by the MRC grant MC PC 19026 to M.P.; by the MRC Grant MC_UU_12016/2 to D.A.; by the Medical Research Council (MC_UU_12016/2) to D.R.A.; by the DFG under Germany's Excellence Strategy (EXC-2189, project ID 390939984 to R.G.); and funding from F. Hoffmann-La Roche and Vir Biotechnology and gifts from The Ron Conway Family and Vir Biotechnology to the Quantitative Biosciences Institute Coronavirus Research Group (QCRG). J.H. was supported by the UCSF Medical Scientist Training Program (T32GM007618); P.F. was supported by the UCSF Medical Scientist Training Program (T32GM007618) and the NIH/NIAID (F30Al143401); M.B. was supported by the NCl at the NIH (F32CA239333); H.T.K. was supported by the NIH (K99GM138753); K.H. was supported by the National Science Foundation (1650113); B.T. was supported by the NIH (F32 CA239336); U.S.C. was supported by the National Institute of General Medical Sciences (F32GM137463). The QCRG Structural Biology Consortium has received support from: Quantitative Biosciences Institute, Defense Advance Research Projects Agency (HR0011-19-2-0020 to D.A.A. and K.A.V.; B. S. PI), FastGrants COVID19 grant (K.A.V. PI), Laboratory For Genomics Research (O. S. R. PI) and Laboratory For Genomics Research (R.M.S. PI). Author contributions: The following authors designed and conceptualized the study: A.A.P., A.G-S., C.F.B., D.E.G., H.B., J.A.R., J.Ba., J.H., K.A.V., K.O., M.B., M.V., N.J.K., O.S.R., P.B., R.G., R.M.K. The following authors performed experiments or data acquisition: A.A.P., A.Du., A.G., A.J.H., A.L.L., A.R., A.R.W., A.S.J., A.S.S-S., A.V-G., B.T., C..J.H., C.Ab., C.Ba., C.F.B., C.J., C.K., C.R.S., D.B., D.E.G., D.K., D.L.S., D.M., D.M.W., D.R.A., D.S., E.M., E.Pe., E.Pu., E.R., E.W.T., F.Be., F.Br., G.J., G.K., H.F., H.L., I.B-H., I.Du., J.A.R., J.A.S., J.Ba., J.C-S., J.H., J.Lo., J.O., J.X., J.Z.G., K.A.B., K.C.K., K.Ho., K.M.W., L.M., M.A.K., M.B., M.B.F., M.Ch., M.C.M., M.D., M.El., M.Gu., Ma.Mo., M.M.K., Ma.McG., Mi.McG., M.U., M.V., N.G., N.Jo., P.B., P.D., P.F., R.H., R.Ha., R.J.K., R.M.K., R.R., R.To., S.G.W., S.P., S.Ra., S.U., S.W., St. W., T.Ke., T.M., T.V., T.W.O., V.V.R., Y.S., Z.Z. The following authors conducted formal data analysis: A.A.P., A.Du., A.J.H., A.L.L., A.R.W., A.S., A.S.J., A.S.S-S., A.V-G., B.S., C..J.H., C.Ab., C.F.B., C.K., D.B., D.E.G., D.L.S., D.M., D.M.W., D.S., E.M., E.Pe., E.W.T., G.J., G.K., H.B., I.Du., J.A.R., J.A.S., J.Ba., J.H., Ji.P., K.A.V., K.C.K., K.M.W., K.O., K.R.H., K.S.P., L.M., M.A.K., M.B., M.B.F., M.Ca., M.C.M., M.Ec., M.Gh., M.Gu., M.J.O., M.M.K., Ma.McG., M.V., N.Jo., N.Ju., O.S.R., P.B., R.G., R.H., R.M.K., R.R., S.P., S.U., S.W., T.A.T., T.I., T.V., T.W.O., U.R., V.V.R., Yu.Z., Z.C., Z.Z. The following authors supervised or managed research: A.A.P., A.F., A.G-S., A.M., A.S., B.S., C.Ab., C.F.B., D.A.A., D.E.G., D.L.S., J.A.R., J.H., J.M.F., J.S.F., K.A.V., K.Ho., K.M.S., K.M.W., K.O., K.S.P., M.B., M.B.F., M.Ch., M.O., M.P., M.V., N.Jo., N.Ju., N.J.K., O.S.R., P.B., R.G., R.H., R.M.K., T.I., T.Ko., Y.C.. The following authors raised funds for these efforts: A.A.P., A.G-S., B.S., C.F.B., D.A.A., D.E.G., D.R.A., J.M.F., K.M.S., K.O., K.S.P., M.P., M.V., N.Jo., N.J.K., O.S.R., P.B., R.G., T.I. The following authors drafted the original manuscript: A.A.P., B.S., C.Ab., D.E.G., D.L.S., H.B., J.A.R., J.Ba., J.H., K.A.V., K.Ha., K.M.S., K.O., K.R.H., K.S.P., M.A.K., M.B., M.Ca., M.Ec., M.M.K., M.O., M.P., M.So., N.J.K., O.S.R., P.B., P.H., R.M.K., T.A.T., U.R., V.V.R., Z.Z.C.N. The structural biology portion of this work was performed by the Quantitative Biosciences Institute Coronavirus Research Group Structural Biology Consortium. Listed below are the contributing members of the consortium listed by teams in order of team relevance to the published work. The team leads are listed first (responsible for 
organization and the experimental design utilized within each team), then the rest of team members are listed alphabetically. Bacterial expression team: A. D., M. G., E. W. T., J. C., L. D., S. F., M. J., H. T. K., V. L. L., Y. L., M. L., G. E. M., J. P., A. C. T., Z. Y., F. Z., Y. Z. Protein purification team: M. M., T. W. O., S. P., C. M. A., C. M. C., B. F., M. G., K. K., J. P., J. K. P., K. S., T. K. M. T. CryoEM grid freezing/collection team: C. M. A., A. F. B., G. E. M., C. P., A. N. R., M. S., J. R. B., M. G., F. L., K. E. L., A. M., F. M., J. P., T. H. P., Jr., S. P., A. M. S., P. V. T., F. W., Z. Y. CryoEM data processing team: A. F. B., M. S. D., G. E. M., H. C. N., A. N. R., D. A., J. R. B., M. G. C., C. M. C., U. S. C., D. D., B. F., M. G., N. H., M. J., F. L., J. L., Y. L., J. P., T. H. P., Jr., S. P., S. S., R. T., D. T., E. T., K. Z., F. Z.. Mammalian cell expression team: C. B., M. G. C., D. D., C. N., A. M. S., J. Z., C. M. A., A. B., N. H., Y. L., P. N., C. P., M. S., S. S., K. S., R. T., T. K. M. T., N. W. Crystallography team: N. H., H. T. K.I, U. S.-G., I. D. Y., J. B., I. D., X. L. Infrastructure team: D. B., A. J., A. J., L. M., M. T., E. T.. Leadership team: O. S. R., K. A. V., D. A. A., Y. C., J. S. F., A. F., N. J., T. K., N. J. K., A. M., D. R. S., R. M. S. All authors edited the manuscript. Competing interests: The Krogan Laboratory has received research support from Vir Biotechnology and F. Hoffmann-La Roche. Trey Ideker is the co-founder of Data4Cure, Inc. with an equity interest, and he has a funded sponsored research agreement from Ideaya BioSciences, Inc., with an equity stake. The García-Sastre Laboratory has received research support from Pfizer, Senhwa Biosciences and 7Hills Pharma and Adolfo García-Sastre has consulting agreements for the following companies involving cash and/or stock: Vivaldi Biosciences, Contrafect, 7Hills Pharma, Avimex, Valneva, Accurius and Esperovax. Kevan Shokat has consulting agreements for the following companies involving cash and/or stock compensation: Black Diamond Therapeutics, BridGene Biosciences, Denali Therapeutics, Dice Molecules, eFFECTOR Therapeutics, Erasca, Genentech/Roche, Janssen Pharmaceuticals, Kumquat Biosciences, Kura Oncology, Merck, Mitokinin, Petra Pharma, Qulab Inc. Revolution Medicines, Type6 Therapeutics, Venthera, Wellspring Biosciences (Araxes Pharma). Jeremy A. Rassen is an employee and shareholder of Aetion, Inc., a company that makes software for the analysis of real-world data. Andrew R. Weckstein is an employee and shareholder of Aetion, Inc., a company that makes software for the analysis of real-world data. Reyna J. Klesh is an employee of HealthVerity, a company that links and de-identifies real-world data. James Fraser is a founder of Keyhole Therapeutics and a shareholder of Relay Therapeutics and Keyhole Therapeutics. The Fraser laboratory has received sponsored research support from Relay Therapeutics. Kevin Holden, Jared Carlson-Stevermer, Jennifer Oki and Travis Maures are employees and shareholders of Synthego Corporation. Aetion holds patents including U.S. Pat. No. 9,378,271; other patents pending. Data and materials availability: Further information and requests for resources and reagents should be directed to and will be fulfilled by Nevan J. Krogan (Nevan.Krogan@ucsf.edu). The mass spectrometry proteomics data have been deposited to the ProteomeXchange Consortium via the PRIDE partner repository with the dataset identifier PXD021588 (95). An interactive version of PPI data can be found at https://kroganlab.ucsf.edu/network-maps. Atomic coordinates and the cryoEM map of the reported Tom70-Orf9b structure have been deposited in the Protein Data Bank under accession code 7KDT and in the Electron Microscopy Data Bank under accession code EMD-22829. Expression vectors used in this study are readily available from the authors for biomedical researchers and educators in the non-profit sector. The Aetion Evidence Platform used for the clinical analysis is available under license from Aetion, New York, NY. To protect patient privacy, data used in real-world analyses are available for inspection by qualified researchers under confidentiality and third-party agreements with Aetion and/or HealthVerity. This work is licensed under a Creative Commons Attribution 4.0 International (CC BY 4.0) license, which permits unrestricted use, distribution, and reproduction in any medium, provided the original work is properly cited. To view a copy of this license, visit

https://creativecommons.org/licenses/by/4.0/. This license does not apply to figures/photos/artwork or other content included in the article that is credited to a third party; obtain authorization from the rights holder before using such material.

\section{SUPPLEMENTARY MATERIALS}

science.sciencemag.org/cgi/content/full/science.abe9403/DC1

QCRG Structural Biology Consortium Author List
Zoonomia Consortium Author List

Figs. S1 to S25

Tables S1 to S15

Reference (96)

MDAR Reproducibility Checklist

Movie S1

24 September 2020; accepted 12 October 2020

Published online 15 October 2020

10.1126/science.abe9403 


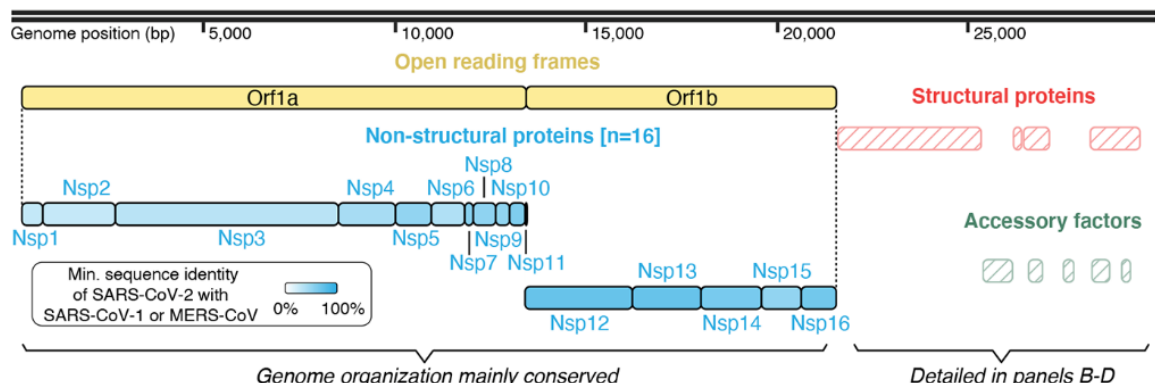

B

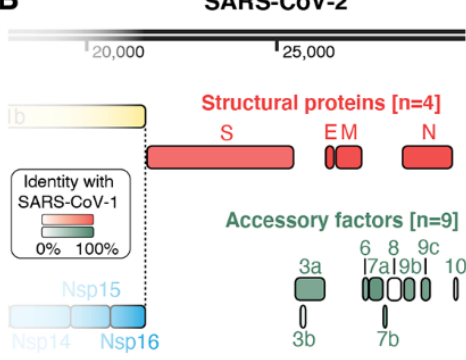

C

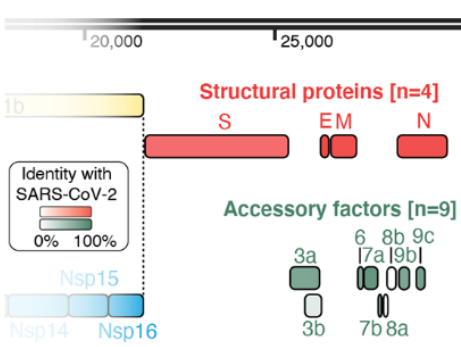

D

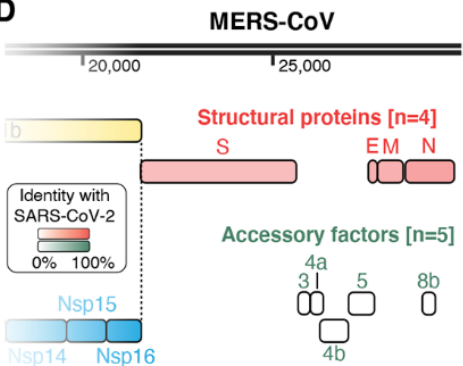

E

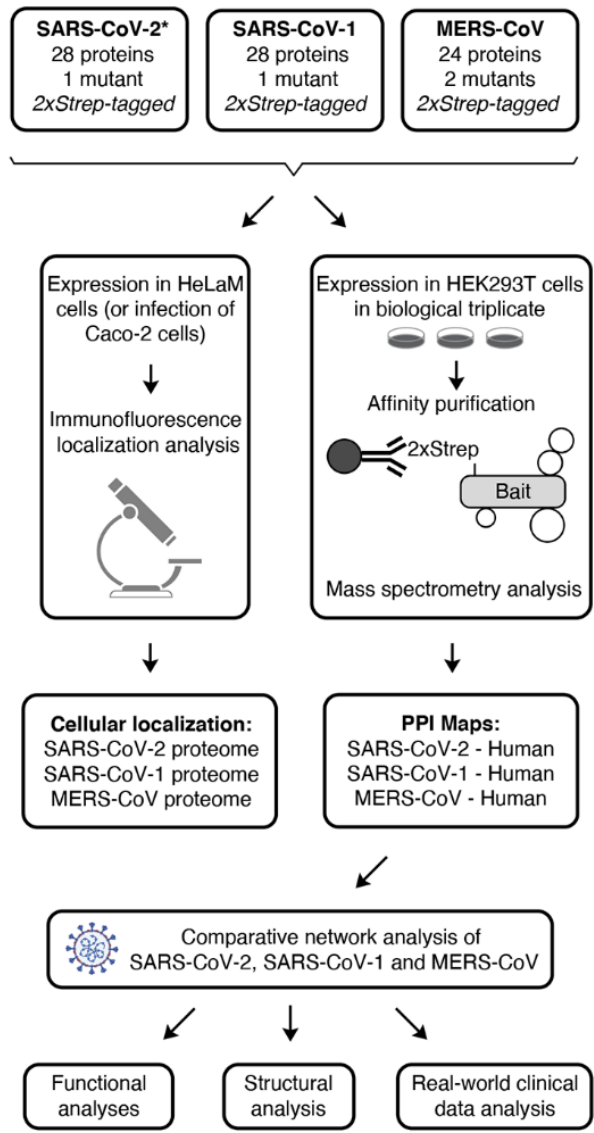

Fig. 1. Coronavirus genome annotations and integrative analysis overview. (A) Genome annotation of SARS-CoV-2, SARS-CoV-1 and MERS-CoV with putative protein coding genes highlighted. Intensity of filled color indicates the lowest sequence identity between SARS-CoV-2 and SARS-CoV-1 or SARS-CoV-2 and MERS. (B to D) Genome annotation of structural protein genes for SARS-CoV-2 (B), SARS-CoV-1 (C), and MERS-CoV (D). Color intensity indicates sequence identity to specified virus. (E) Overview of comparative coronavirus analysis. Proteins from SARS-CoV-2, SARS-CoV-1 and MERS-CoV were analyzed for their protein interactions and subcellular localization, and these data were integrated for comparative host interaction network analysis, followed by functional, structural and clinical data analysis for exemplary virusspecific and pan-viral interactions. *The SARS-CoV-2 interactome was previously published in a separate study (5). SARS = both SARS-CoV-1 and SARS-CoV-2; MERS = MERS-CoV; Nsp = non-structural protein; Orf = open reading frame. 

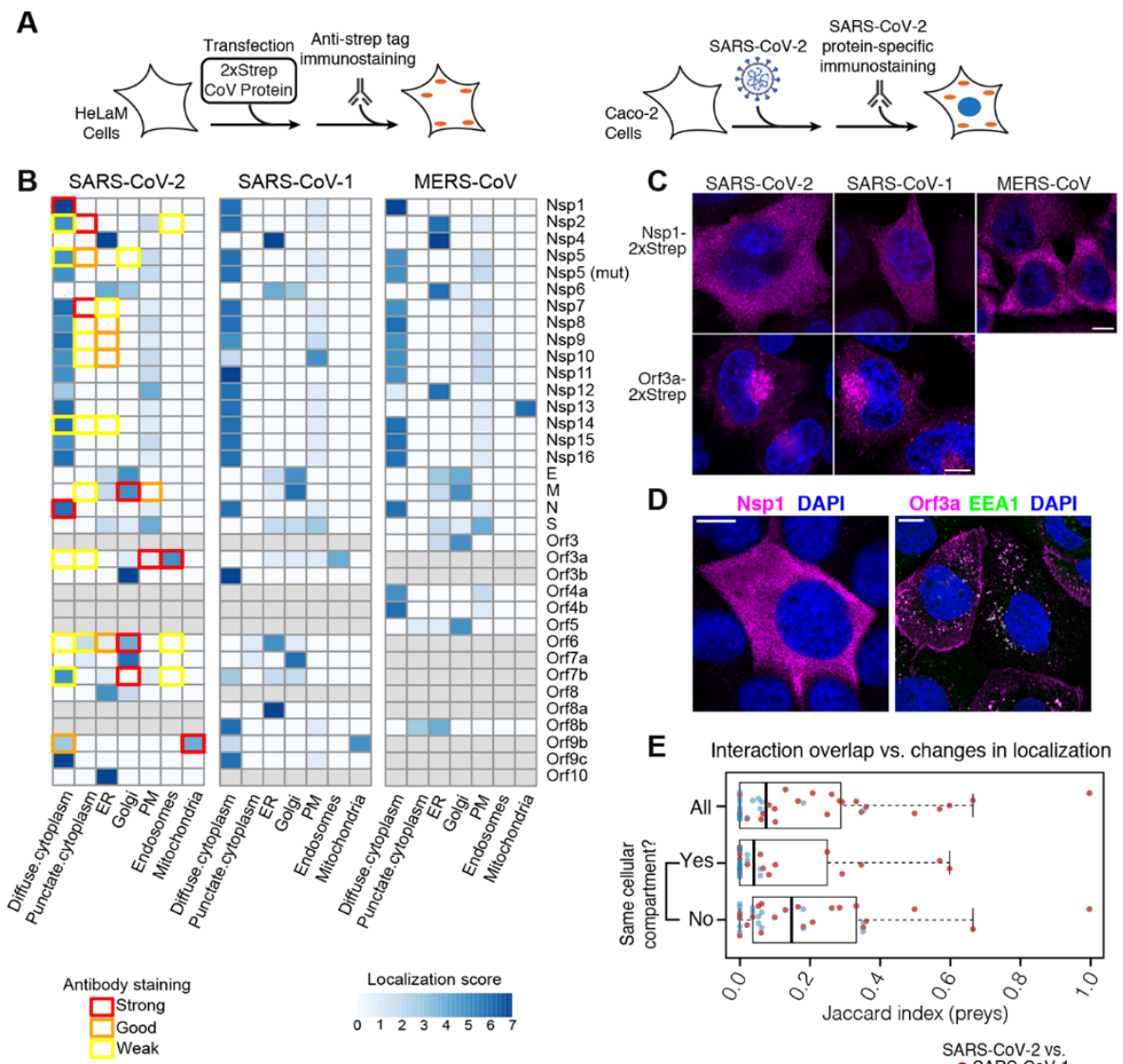

D

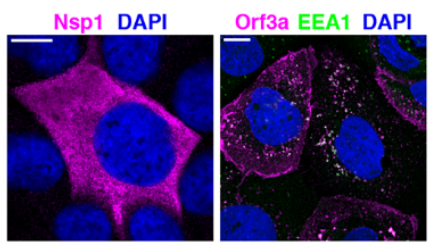

E Interaction overlap vs. changes in localization

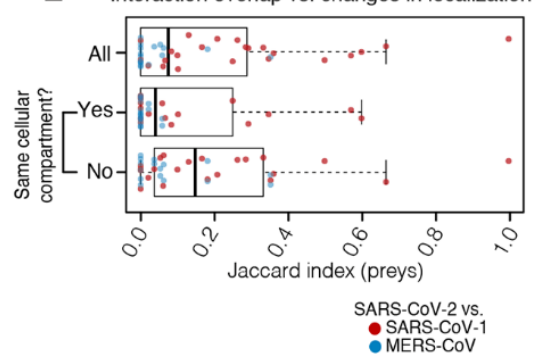

Fig. 2. Coronavirus protein localization analysis. (A) Overview of experimental design to determine localization of Strep-tagged SARS-CoV-2, SARS-CoV-1, and MERS-CoV proteins in HeLaM cells (left) or of viral proteins upon SARS-CoV-2 infection in Caco-2 cells (right). (B) Relative localization for all coronavirus proteins across viruses expressed individually (blue color bar; * indicates viral proteins of high sequence divergence) or in SARS-CoV-2 infected cells (colored box outlines). (C and D) Localization of Nsp1 and Orf3a expressed individually (C) or during infection (D); for representative images of all tagged constructs and viral proteins imaged during infection see figs. S8 to S14 and fig. S15 respectively. (E) Prey overlap per bait measured as Jaccard index comparing SARS-CoV-2 vs. SARS-CoV-1 (red dots) and SARS-CoV-2 vs. MERS-CoV (blue dots) for all viral baits (All), viral baits found in the same cellular compartment (Yes) and viral baits found in different compartments (No). C-D, Scale bars $=10 \mu \mathrm{m}$. 


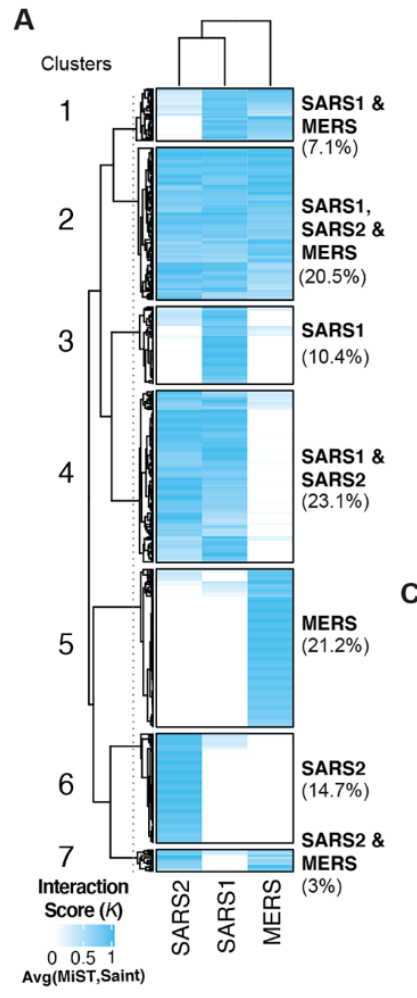

E

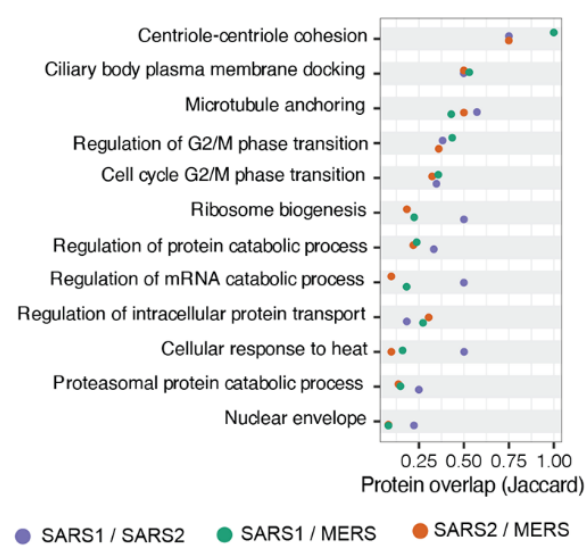

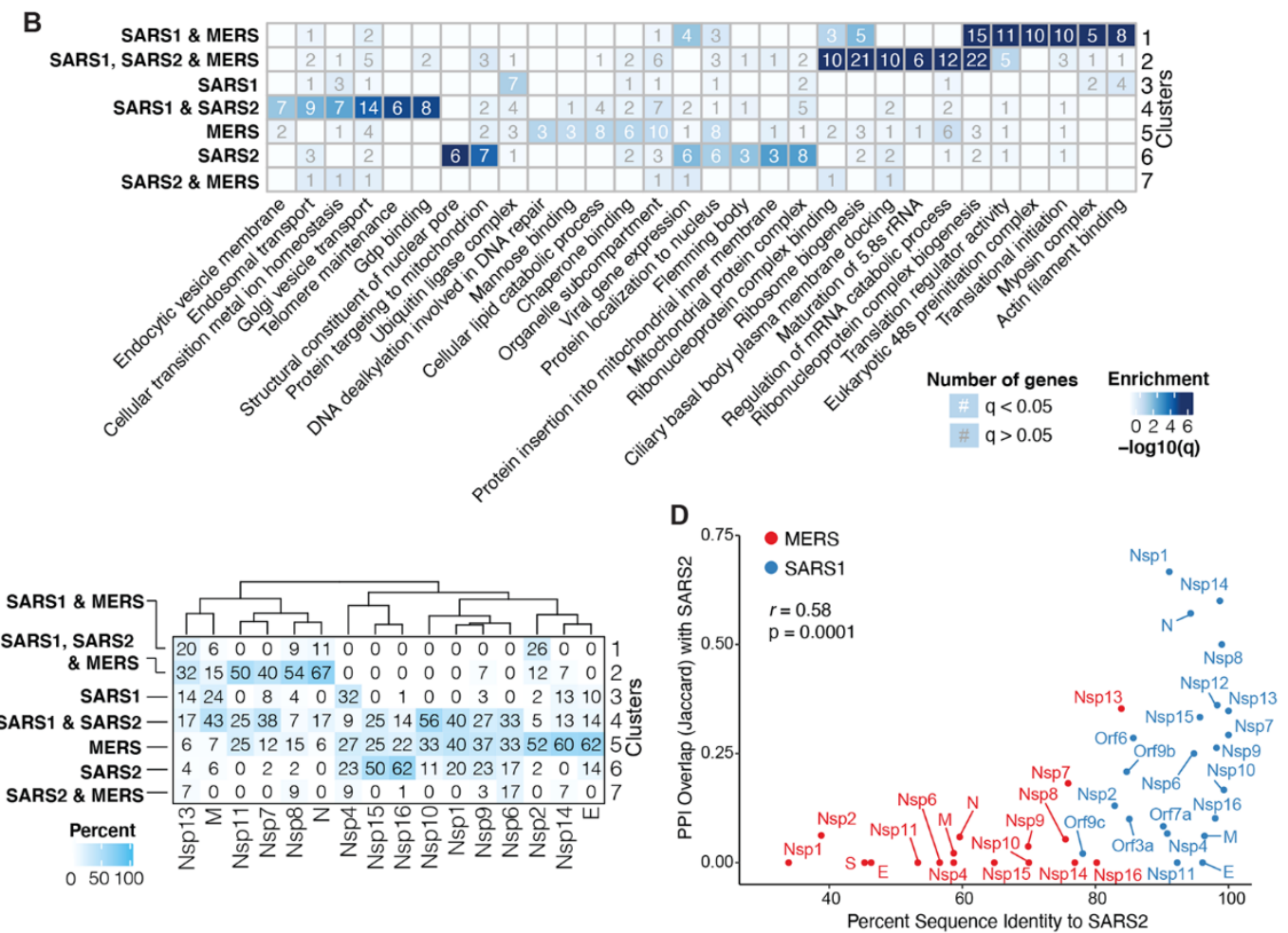

$\mathbf{F}$

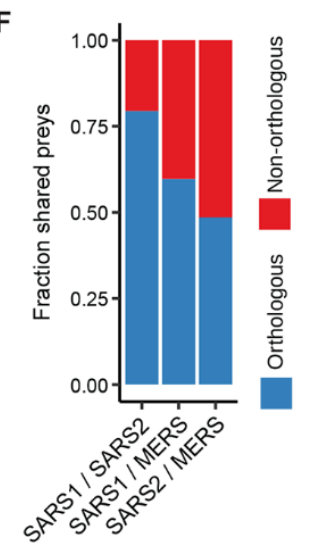

G

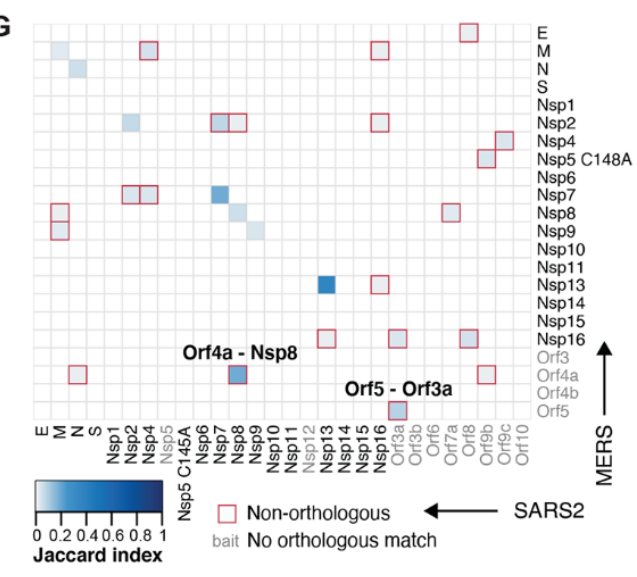

Fig. 3. Comparative analysis of coronavirus-host interactomes. (A) Clustering analysis (k-means) of interactors from SARS-CoV-2, SARS-CoV-1, and MERS-CoV weighted according to the average between their MIST and Saint scores (interaction score $K$ ) and percentages of total interactions. Included are only viral protein baits represented amongst all three viruses and interactions that pass the high-confidence scoring threshold for at least one virus. Seven clusters highlight all possible scenarios of shared versus unique interactions. (B) GO enrichment analysis of each cluster from A, with the top six most significant terms per cluster. Color indicates $-\log 10(q)$ and number of genes with significant $(q<0.05$; white) or nonsignificant enrichment ( $q>0.05$; grey) is shown. (C) Percentage of interactions for each viral protein belonging to each cluster identified in A. (D) Correlation between protein sequence identity and PPI overlap (Jaccard index) comparing SARS-CoV-2 and SARS-CoV-1 (blue) or MERS-CoV (red). Interactions for PPI overlap are derived from the final thresholded list of interactions per virus. (E) GO biological process terms significantly enriched $(q<0.05)$ for all three virus PPIs with Jaccard index indicating overlap of genes from each term for pairwise comparisons between SARS-CoV-1 and SARS-CoV-2 (purple), SARS-CoV-1 and MERS-CoV (green) and SARS-CoV-2 and MERS-CoV (orange). (F) Fraction of shared preys between orthologous (blue) versus non-orthologous (red) viral protein baits. (G) Heatmap depicting overlap in PPIs (Jaccard index) between each bait from SARS-CoV-2 and MERS-CoV. Baits in grey were not assessed, do not exist, or do not have highconfidence interactors in the compared virus. Non-orthologous bait interactions are highlighted with a red square. GO $=\mathrm{Gene}$ Ontology; PPI = protein-protein interaction; SARS2 = SARS-CoV-2; SARS1 = SARS-CoV-1; MERS = MERS-CoV. 

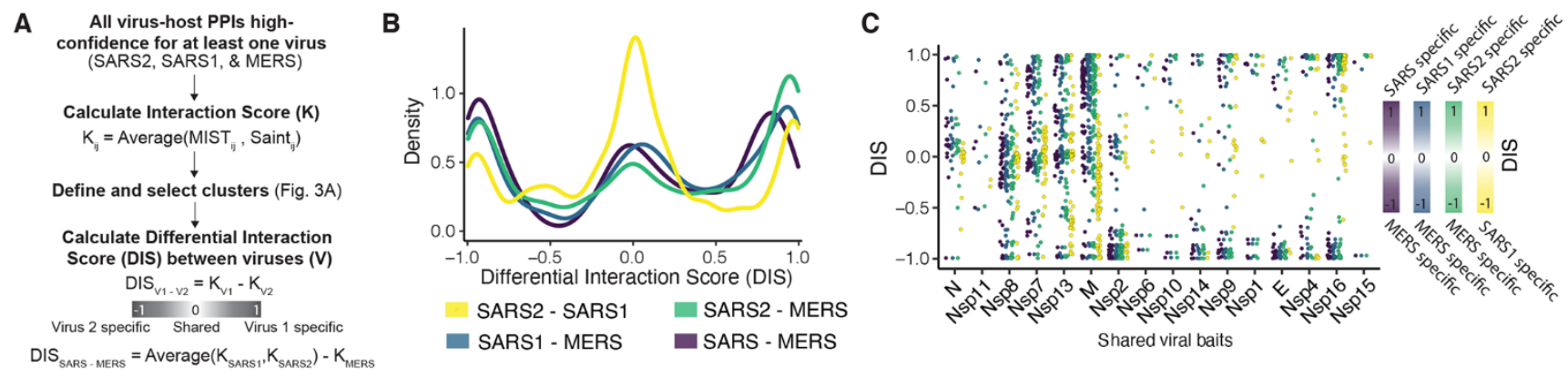

D
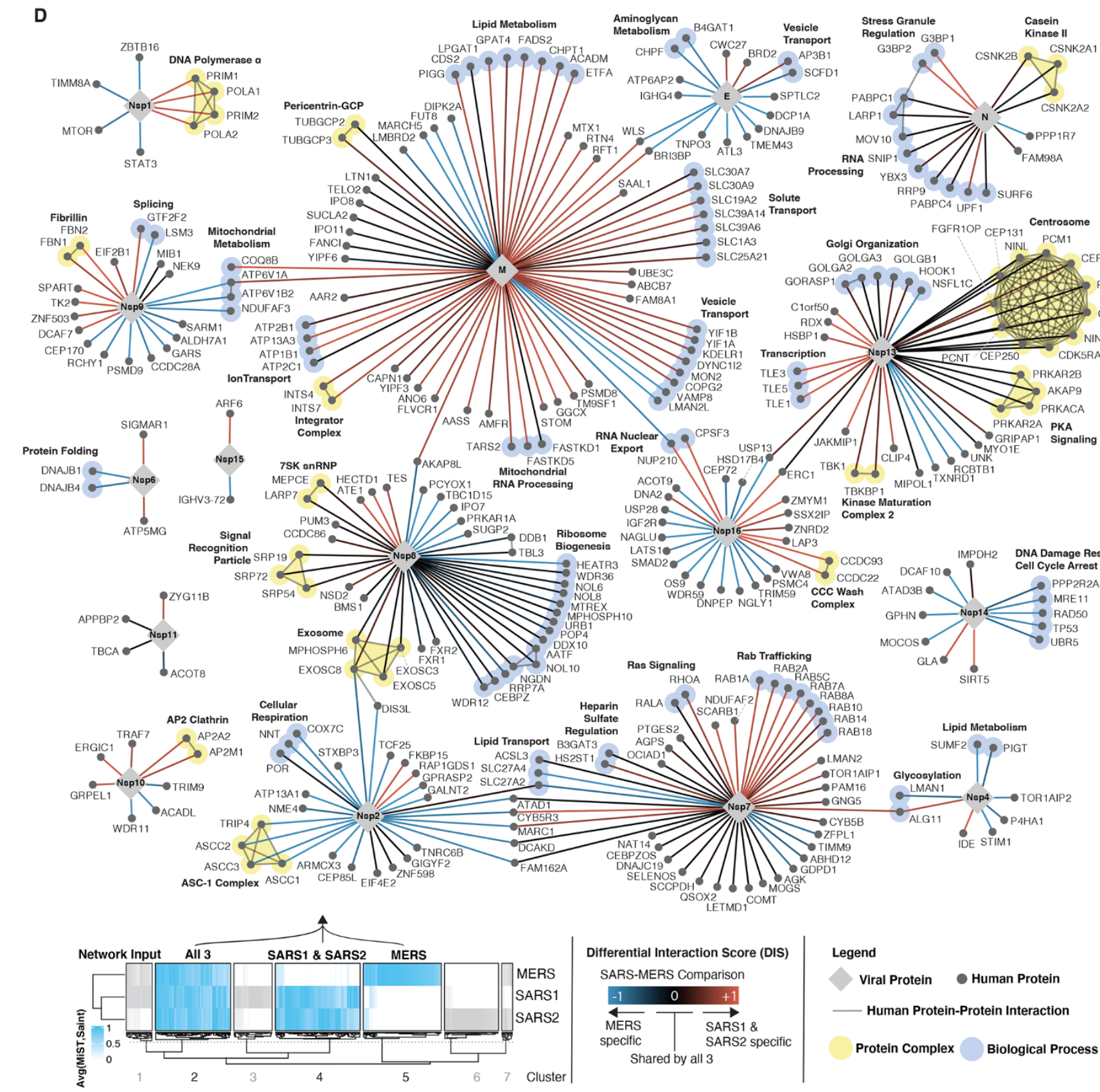
Fig. 4. Comparative differential interaction analysis reveals shared virus-host interactions. (A) Flowchart depicting calculation of differential interactions scores (DIS) using the average between the Saint and MIST scores between every bait $(i)$ and prey $(j)$ to derive interaction score $(K)$. The DIS is the difference between the interaction scores from each virus. The modified DIS (SARS-MERS) compares the average $K$ from SARSCoV-1 and SARS-CoV-2 to that of MERS-CoV (see Methods). Only viral bait proteins shared between all three viruses are included. (B) Density histogram of the DIS for all comparisons. (C) Dot plot depicting the DIS of interactions from viral bait proteins shared between all three viruses, ordered left-to-right by the mean DIS per viral bait. (D) Virus-human protein-protein interaction map depicting the SARS-MERS comparison (purple in Fig. 4, B and C). The network depicts interactions derived from cluster 2 (all 3 viruses), cluster 4 (SARS-CoV-1 and SARS-CoV-2), and cluster 5 (MERS-CoV only). Edge color denotes DIS: red, interactions specific to SARS-CoV-1 and SARS-CoV-2 but absent in MERS-CoV; blue, interactions specific to MERS-CoV but absent from both SARS-CoV-1 and SARS-CoV-2; black, interactions shared between all three viruses. Human-human interactions (thin dark grey line), proteins sharing the same protein complexes or biological processes (light yellow or light blue highlighting, respectively) are shown. Host-host physical interactions, protein complex definitions, and biological process groupings are derived from CORUM (39), Gene Ontology (biological process), and manually curated from literature sources. Thin dashed grey lines are used to indicate the placement of node labels when adjacent node labels would have otherwise been obscured. DIS = differential interactions score; SARS2 = SARS-CoV-2; SARS1 = SARS-CoV-1; MERS = MERS-CoV; SARS = both SARS-CoV-1 and SARS-CoV-2. 

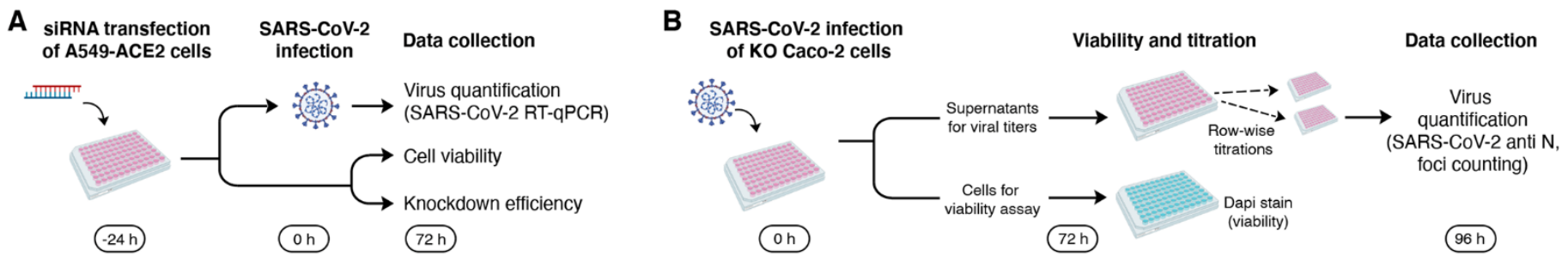

C

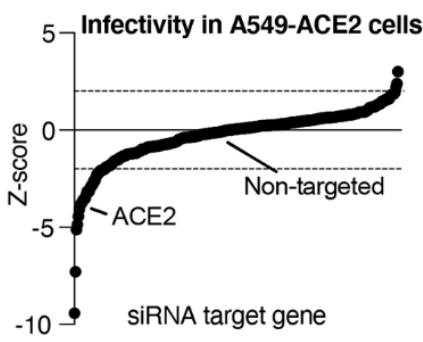

D

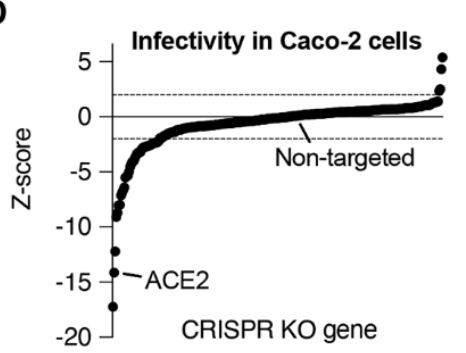

E
Infectivity in A549-ACE2

and Caco-2 cells
$\mathbf{F}$
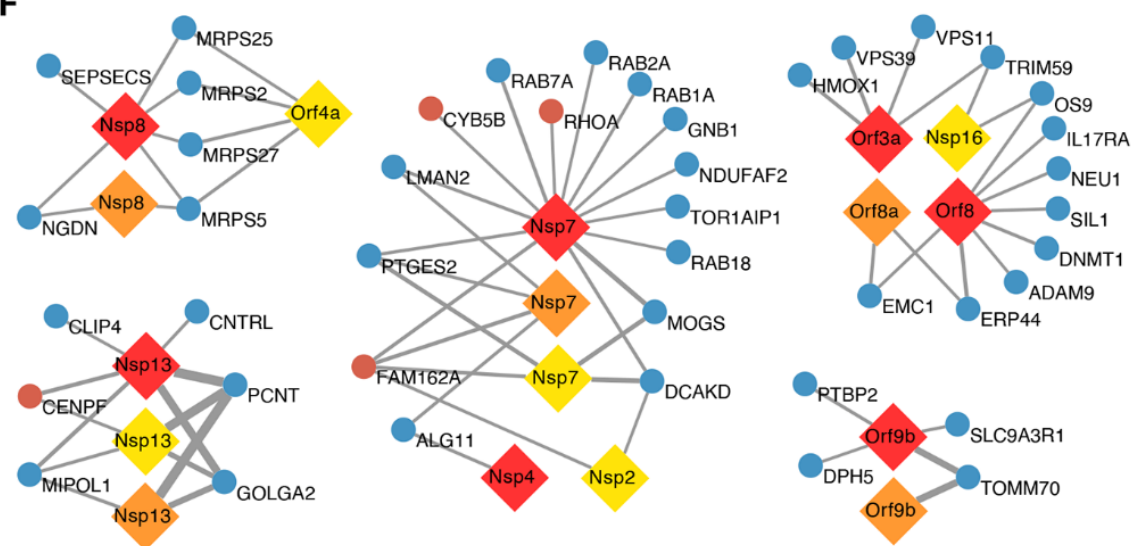

\section{$-10$}

A549-ACE2

Z-score

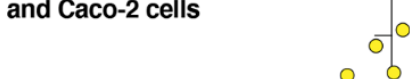

$7_{0}^{10}$
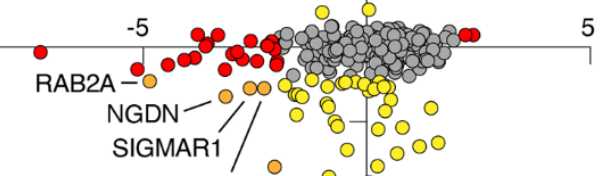

SIGMAR1/ 0

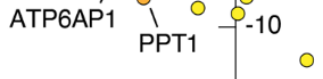
$\begin{array}{ll}\text { ACE2 }^{\circ} & -15^{\circ} \\ \text { Caco-2 Z-score } & -20 \\ & \end{array}$
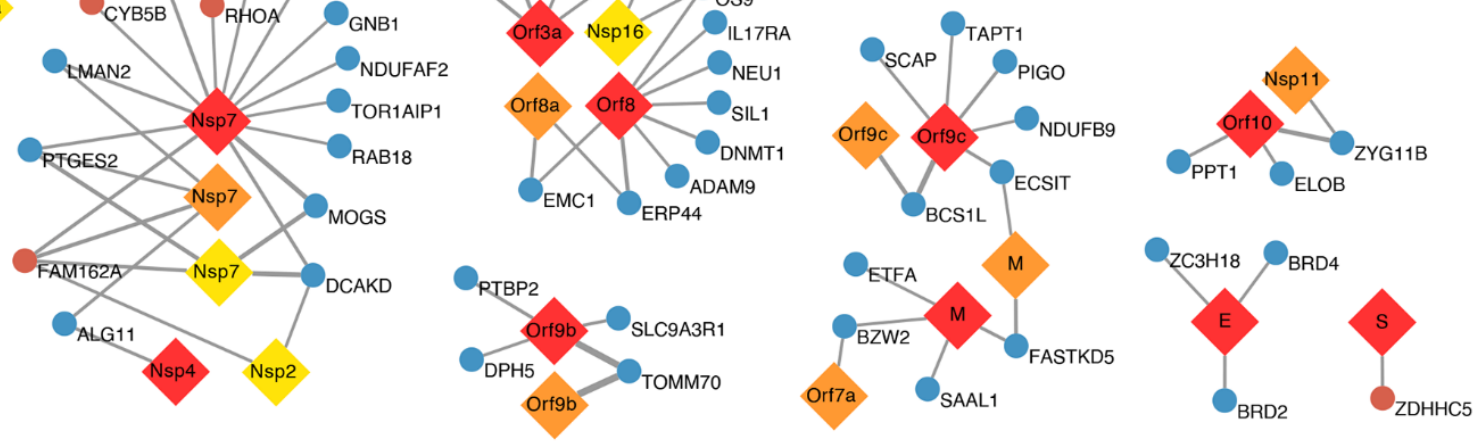

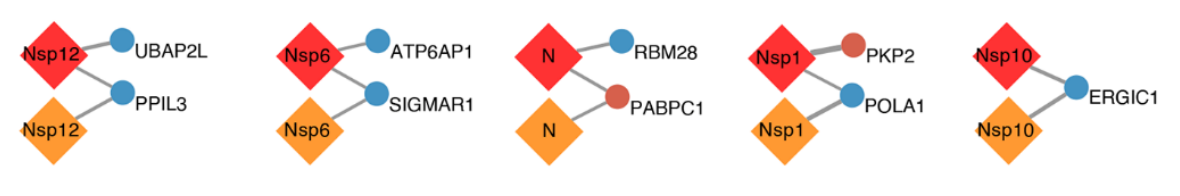

SARS-CoV-2 Protein

SARS-CoV-1 Protein

MERS-CoV Protein

Decreased infectivity in $\mathrm{KD} / \mathrm{KO}$
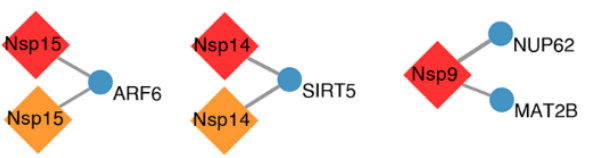

$\uparrow \mathrm{PPI}$ spectral counts 
Fig. 5. Functional interrogation of SARS-CoV-2 interactors using genetic perturbations. (A) A549-ACE2 cells were transfected with siRNA pools targeting each of the human genes from the SARS-CoV-2 interactome, followed by infection with SARS-CoV-2 and virus quantification using RT-qPCR. Cell viability and knockdown efficiency in uninfected cells was determined in parallel. (B) Caco-2 cells with CRISPR knockouts of each human gene from the SARS-CoV-2 interactome were infected with SARS-CoV-2, and supernatants were serially diluted and plated onto Vero E6 cells for quantification. Viabilities of the uninfected CRISPR knockout cells after infection were determined in parallel by DAPI staining. ( $C$ and $D$ ) Plot of results from the infectivity screens in A549-ACE2 knockdown cells (C) and Caco-2 knockout cells (D) sorted by $Z$-score ( $Z<0$, decreased infectivity; $Z>0$ increased infectivity). Negative controls (non-targeting control for siRNA, non-targeted cells for CRISPR) and positive controls (ACE2 knockdown/knockout) are highlighted. (E) Results from both assays with potential hits $(|Z|>2)$ highlighted in red (A549-ACE2), yellow (Caco-2) and orange (both). (F) Pan-coronavirus This work is licensed under a Creative Commons Attribution 4.0 International (CC BY 4.0) license, which permits unrestricted use, distribution, and reproduction in any medium, provided the original work is properly cited. To view a copy of this license, visit https://creativecommons.org/licenses/by/4.0/. This license does not apply to figures/photos/artwork or other content included in the article that is credited to a third party; obtain authorization from the rights holder before using such material.interactome reduced to human preys with significant increase (red nodes) or decrease (blue nodes) in SARS-CoV2 replication upon knockdown/knockout. Viral proteins baits from SARS-CoV-2 (red), SARSCoV-1 (orange) and MERS-CoV (yellow) are represented as diamonds. The thickness of the edge indicates the strength of the PPI in spectral counts. $\mathrm{KD}=$ Knockdown; $\mathrm{KO}=$ Knockout; $\mathrm{PPI}=$ protein-protein interaction. 

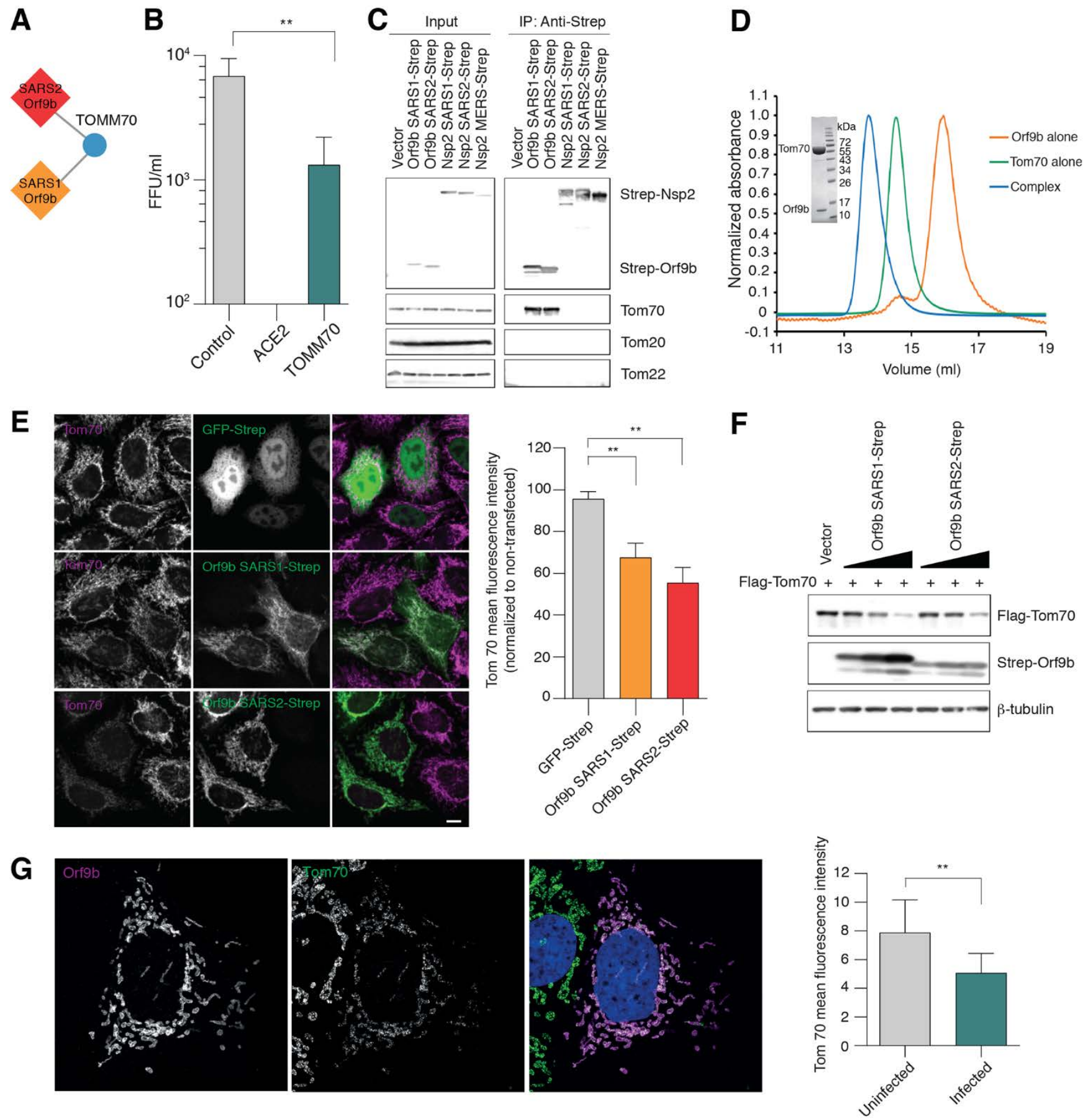

Fig. 6. Interaction between Orf9b and human Tom70. (A) Orf9b-Tom70 interaction is conserved between SARSCoV-1 and SARS-CoV-2. (B) Viral titers in Caco-2 cells after CRISPR knockout of TOMM70 or controls. (C) Coimmunoprecipitation of endogenous Tom70 with Strep-tagged Orf9b from SARS-CoV-1 and SARS-CoV-2, Nsp2 from SARS-CoV-1, SARS-CoV-2 and MERS-CoV, or vector control in HEK293T cells. Representative blots of whole cell lysates and eluates after IP are shown. (D) Size exclusion chromatography traces (10/300 S200 Increase) of Orf9b alone, Tom70 alone and co-expressed Orf9b-Tom70 complex purified from recombinant expression in $E$. coli. Insert shows SDS-PAGE of the complex peak indicating presence of both proteins. (E) Immunostainings for Tom70 in HeLaM cells transfected with GFP-Strep and Orf9b from SARS-CoV-1 and SARS-CoV-2 (left) and mean fluorescence intensity \pm SD values of Tom70 in GFP-Strep and Orf9b expressing cells (normalized to nontransfected cells; right). (F) Flag-Tom70 expression levels in total cell lysates of HEK293T cells upon titration of cotransfected Strep-Orf9b from SARS-CoV-1 and SARS-CoV-2. (G) Immunostaining for Orf9b and Tom70 in Caco-2 cells infected with SARS-CoV-2 (left) and mean fluorescence intensity \pm SD values of Tom70 in uninfected and SARS-CoV-2 infected cells (right). SARS2 = SARS-CoV-2; SARS1 = SARS-CoV-1; MERS = MERS-CoV; IP = immunoprecipitation. ${ }^{* *} \mathrm{p}<0.05$. B, E, G, Student's $t$ test. E, scale bar $=10 \mu \mathrm{m}$. 
A

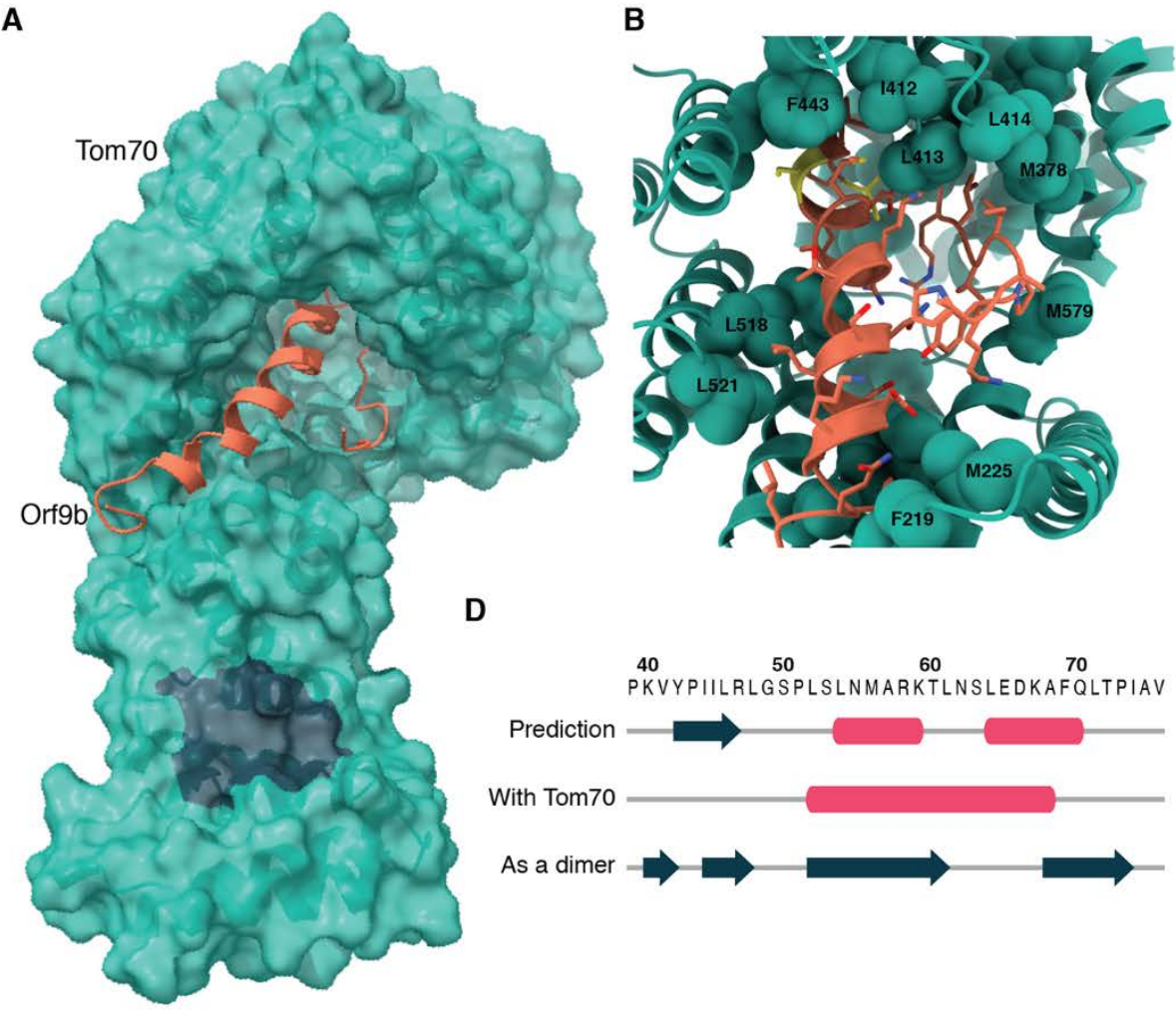

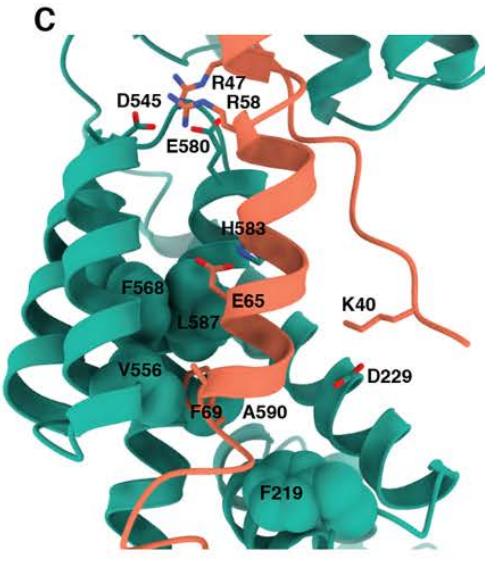

E

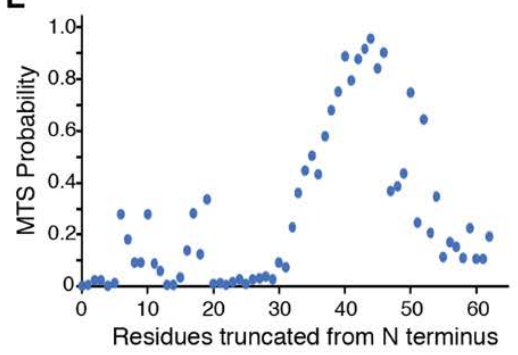

Fig. 7. CryoEM structure of Orf9b-Tom70 complex reveals Orf9b adopting a helical fold and binding at the substrate recognition site of Tom70. (A) Surface representation of the Orf9b-Tom70 structure. Tom70 is depicted as molecular surface in green, Orf9b is depicted as ribbon in orange. Region in charcoal indicates Hsp70/Hsp90 binding site on Tom70. (B) Magnified view of Orf9b-Tom70 interactions with interacting hydrophobic residues on Tom70 indicated and shown in spheres. The two phosphorylation sites on Orf9b, S50 and S53, are shown in yellow. (C) lonic interactions between Tom70 and Orf9b are depicted as sticks. Highly conserved residues on Tom70 making hydrophobic interactions with Orf9b are depicted as spheres. (D) Diagram depicting secondary structure comparison of Orf9b as predicted by JPred server, as visualized in our structure, or as visualized in the previously-crystallized dimer structure (PDB:6Z4U) (16). Pink tubes indicate helices, charcoal arrows indicate beta strands, amino acid sequence for the region visualized in the cryoEM structure is shown on top. (E) Predicted probability of possessing an internal MTS as output by TargetP server by serially running N-terminally truncated regions of SARS-CoV-2 Orf9b. Region visualized in the cryoEM structure (amino acids 39-76) overlaps with the highest internal MTS probability region (amino acids 40-50). MTS = mitochondrial targeting signal. 
A

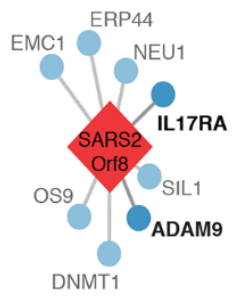

C

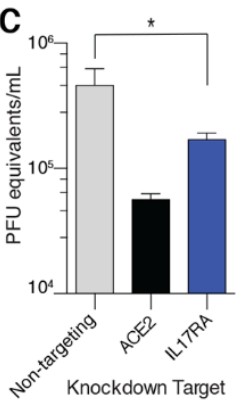

B

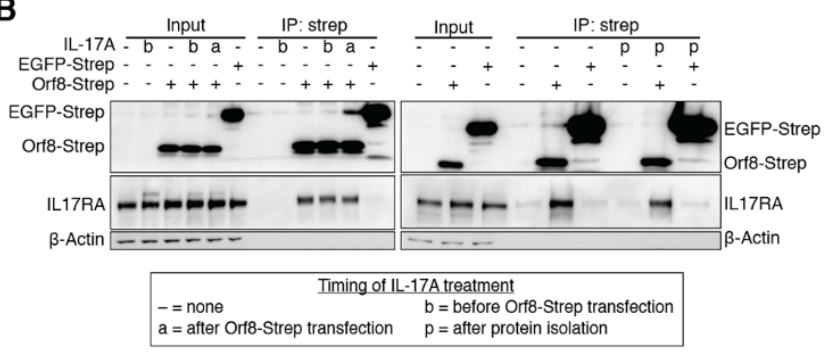

D

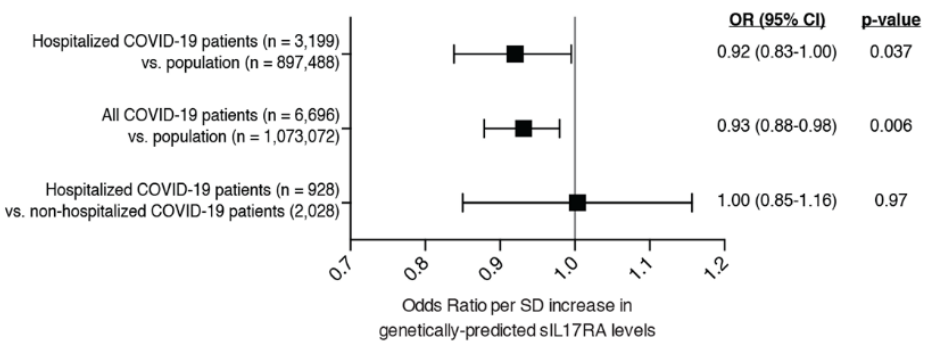

Fig. 8. SARS-CoV-2 Orf8 and functional interactor IL17RA are linked to viral outcomes. (A) IL17RA and ADAM9 are functional interactors of SARS-CoV-2 Orf8. Only interactors identified in the genetic screening are shown. (B) Co-immunoprecipitation of endogenous IL17RA with Streptagged Orf8 or EGFP with or without IL-17A treatment at different times. Overexpression was done in HEK293T cells. (C) Viral titer after IL17RA or control knockdown in A549-ACE2 cells. (D) Odds ratio of membership in indicated cohorts by genetically-predicted sIL17RA levels. SARS2 = SARS-CoV-2; IP = immunoprecipitation; $\mathrm{SD}=$ standard deviation; $\mathrm{OR}=$ odds ratio; $\mathrm{Cl}=$ confidence interval; sIL17RA = soluble IL17RA. ${ }^{*}=p<0.05$. $C$, unpaired $t$ test. Error bars in C indicate SD; in D they indicate $95 \% \mathrm{Cl}$. 
A
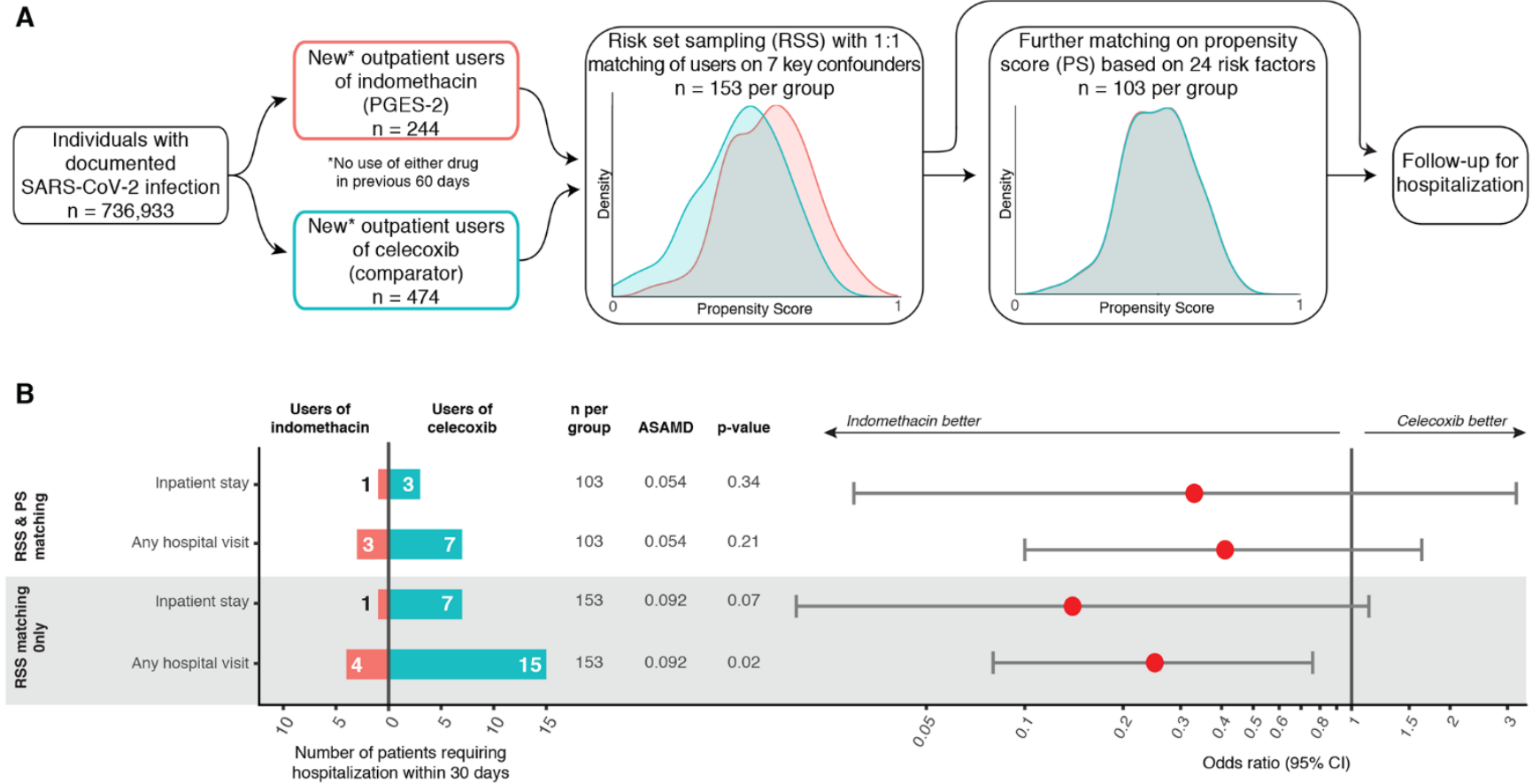

C

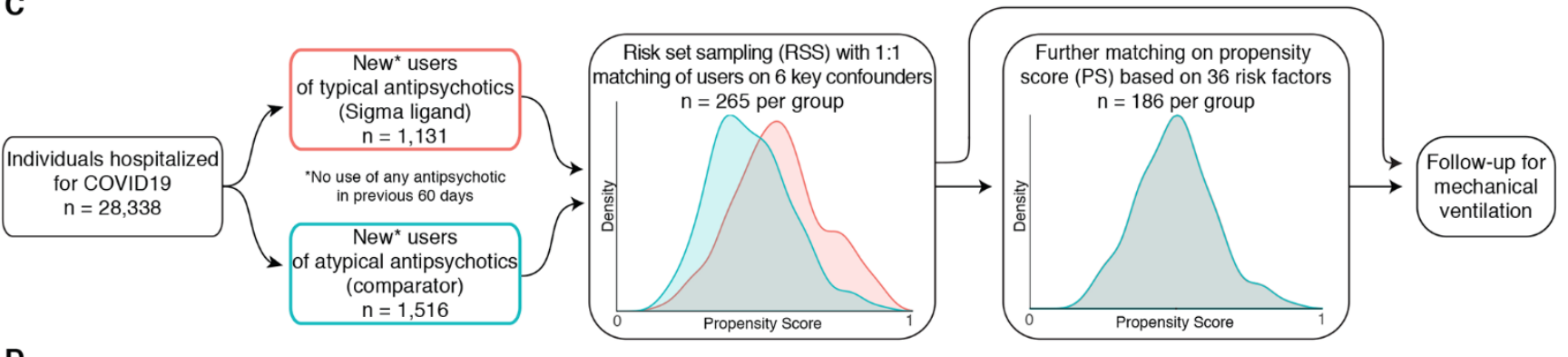

D

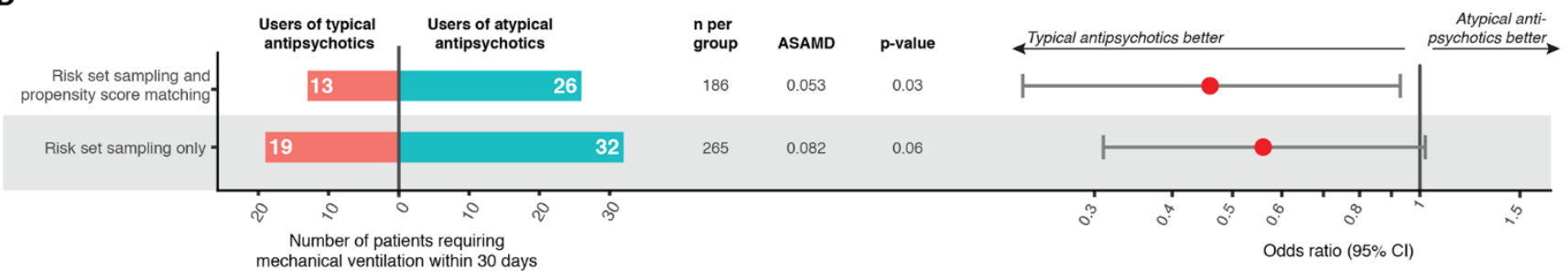

Fig. 9. Real-world data analysis of drugs identified through molecular investigation support their antiviral activity. (A) Schematic of retrospective real-world clinical data analysis of indomethacin use for outpatients with SARS-CoV-2. Plots show distribution of propensity scores for all included patients (red, indomethacin users; blue, celecoxib users). For a full list of inclusion, exclusion, and matching criteria see Methods and table S11. (B) Effectiveness of indomethacin vs. celecoxib in patients with confirmed SARS-CoV-2 infection treated in an outpatient setting. Average standardized absolute mean difference (ASAMD) is a measure of balance between indomethacin and celecoxib groups calculated as the mean of the absolute standardized difference for each propensity score factor (table S11); $p$-value and odds ratios with $95 \% \mathrm{Cl}$ are estimated using the Aetion Evidence Platform r4.6. No ASAMD was greater than 0.1. (C) Schematic of retrospective real-world clinical data analysis of typical antipsychotic use for inpatients with SARS-CoV-2. Plots show distribution of propensity scores for all included patients (red, typical users; blue, atypical users). For a full list of inclusion, exclusion, and matching criteria see Methods and table S11. (D) Effectiveness of typical vs. atypical antipsychotics among hospitalized patients with confirmed SARS-CoV-2 infection treated inhospital. Average standardized absolute mean difference (ASAMD) is a measure of balance between typical and atypical groups calculated as the mean of the absolute standardized difference for each propensity score factor (table S11); p-value and odds ratios with 95\% Cl are estimated using the Aetion Evidence Platform r4.6. No ASAMD was greater than 0.1. 